\title{
Doubts about the Model and Optimal Policy
}

\section{Anastasios G. Karantounias}

\section{Working Paper 2020-12 \\ July 2020}

\begin{abstract}
This paper analyzes optimal policy in setups where both the leader and the follower have doubts about the probability model of uncertainty. I illustrate the methodology in two environments: a) an industry populated with a large firm and many small firms in a competitive fringe, where both types of firms doubt the probability model of demand shocks, and b) a general equilibrium economy, where a policymaker taxes linearly the labor income of a representative household in order to finance an exogenous stream of stochastic spending shocks. The policymaker can distrust the probability model of spending shocks more, the same, or less than the household. Whenever there are doubts about the model, cautious agents form endogenous worstcase beliefs by assigning high probability on low profitability or low-utility events. There are two forces that shape optimal policy results: the manipulation of the endogenous beliefs of the follower to the benefit of the leader, and the discrepancy (if any) in the pessimistic beliefs between the leader and the follower. Depending on the application, the leader may amplify or mitigate the worst-case beliefs of the follower.
\end{abstract}

JEL classification: D80, E62, H21, H63

Key words: model uncertainty, ambiguity aversion, multiplier preferences, misspecification, robustness, martingale, monopolist, competitive fringe, demand uncertainty, Ramsey taxation

https://doi.org/10.29338/wp2020-12

The author thanks Thomas J. Sargent for introducing him to this research agenda and providing constant encouragement and inspiration. The author is also grateful to Lars Peter Hansen and to the late David K. Backus for comments. An earlier version of this paper was titled "Doubts about the model and optimal taxation." The views expressed here are those of the author and not necessarily those of the Federal Reserve Bank of Atlanta or the Federal Reserve System. Any remaining errors are the author's responsibility.

Please address questions regarding content to Anastasios G. Karantounias, Research Department, Federal Reserve Bank of Atlanta, 1000 Peachtree St. NE, Atlanta, GA 30309, anastasios.karantounias@atl.frb.org.

Federal Reserve Bank of Atlanta working papers, including revised versions, are available on the Atlanta Fed's website at www.frbatlanta.org. Click "Publications" and then "Working Papers." To receive e-mail notifications about new papers, use frbatlanta.org/forms/subscribe. 


\section{Introduction}

This paper analyzes the effects of model uncertainty on the design of optimal policy. Uncertainty is pervasive, hard to quantify and difficult to act upon. Economic agents (households or firms) and policymakers acknowledge that. They recognize that their probability models may be misspecified and are cautious towards this model uncertainty or ambiguity. In this paper, I put both the policymaker and the economic agents on equal footing and allow them to exhibit fears of model misspecification. I lay down the general methodology of setting up policy problems with model uncertainty and study the main forces that emerge.

I study policy under commitment and I allow both the Stackelberg leader and the follower to have fears of misspecification about the probability model of exogenous uncertainty. I use the multiplier preferences of Hansen and Sargent (2001) to express aversion to model ambiguity. In order to illustrate the methodology, I use two separate environments: a) a microeconomic setup of an industry where a large firm faces a competitive fringe a) a macroeconomic setup where a government chooses optimally how to tax the labor income of the representative consumer in order to finance a stream of exogenous government expenditures.

Each of the two environments has its own advantages. The industry problem is sufficiently rich to have both backward-looking and forward-looking constraints. Additionally, this setup allows us to have a richer representation of the pessimistic beliefs that agents form: these beliefs may differ due to either different attitude towards model ambiguity or to different return (profit) functions. The industry problem has also the typical structure of standard monetary policy problems and makes contact also with the industrial organization and demand uncertainty literature.

Turning to the fiscal policy problem, its usefulness lies in two separate aspects: first, it allows to show explicitly how to introduce doubts about the model for both the policymaker and the private sector in a general equilibrium economy. Second, it permits the natural distinction between the case of a benevolent planner, who adopts the welfare criterion of the representative household, and the case of a paternalistic government, which may doubt the model more or less than the household. This freedom in forming the criterion of the policymaker is welcome because, in an environment with subjective uncertainty, it is not clear anymore what the normative welfare criterion should be.

When there is model ambiguity, each of the economy participants forms cautious beliefs based on their objective criterion. A cautious large or competitive firm assigns high probability on low profitability events. Similarly, a government and a representative household assign high probability on low utility events, which are typically associated with high, non-utility providing

spending shocks. Aside from this obvious pessimism, there is an additional angle in optimal policy problems, coming from the fact that the worst-case beliefs of the follower are endogenous. 
As a result, they can be affected, or 'managed' to the benefits of the leader.

To see that, consider the general methodology of the optimal policy problem under commitment. The follower (the competitive fringe or the representative household) tilts its probability assessments towards events with low profitability or low utility. Worst-case beliefs affect the behavior of the follower and determine equilibrium objects. In the industry application, pessimistic price forecasts determine the quantity produced by the competitive fringe, and therefore, the prevailing equilibrium price. In the fiscal policy problem, pessimistic evaluations of contingencies determine the price of state-contingent debt and therefore the tradeoffs between taxing today versus issuing debt.

The leader (large firm or government) understands that these cautious forecasts depend on endogenous objects (profits or utility), and therefore uses its instruments (quantity of the produced good or the labor tax) to properly affect the evolution of profits or utility of the follower. The pessimistic expectation management takes different forms depending on the application: in the industry problem, the large firm has an incentive to make the competitive fringe entertain pessimistic forecasts of demand shocks. Such forecasts lead to reduced fringe production, allowing larger profits for the large firm. Thus, the large firm has an incentive to amplify the pessimism of the fringe. In the optimal taxation application, this result is reversed. The government has an incentive to make new debt cheaper, since this relaxes the government budget and allows lower tax rates in the current period. Exactly because the government sells debt against good times, it has an incentive to increase revenue from debt issuance by making the price of debt against good times higher. This is achieved by making the pessimistic household assign a higher probability on good times. Thus, the government is trying to mitigate the inherent pessimism of the household in order to manipulate properly the price of government debt.

Lastly, the worst-case beliefs of the leader and his disagreement with the follower form an independent channel of influence on optimal policy. This can be seen in a transparent way in the optimal taxation problem of the second part of the paper. In the case of a paternalistic government, the ratio of worst-case beliefs of the household and the government plays an independent role in the design of policy. Depending on the strength of the relative pessimism of the government versus the household's, paternalism can act either in the same direction with, or in the opposite direction to the management of the pessimistic expectations of the follower.

\section{$1.1 \quad$ Related literature}

In this section I want to pay tribute to the intellectual ancestors of the current paper. Model uncertainty and robustness concerns have played a major role in thinking about policy, especially monetary policy.

Most previous work has focused on fears of model misspecification on the side of the policymaker. An example among the earliest conributions is Tetlow and von zur Muehlen (2001), 
who study the effects of unstructured and structured misspecifications on the design of policy rules and address the question of aggressive or attenuated policy responses in light of model uncertainty. Barlevy (2009) and Barlevy (2011) delve further into these issues. Tetlow (2015) puts us in the shoes of an actual policymaker and studies the robustness and performance of various policy rules in forty-six vintages of the FRB/US model of the Federal Reserve. Giannoni (2002) analyzes robust Taylor rules and Giannoni (2007) deals with both shock and parameter uncertainty. Cogley et al. (2008) deals with the intriguing issue of robustness and the motives for learning and experimentation in U.S. policy. ${ }^{1}$

I use the multiplier preferences of Hansen and Sargent (2001), that have been axiomatized by Strzalecki (2011). Hansen and Sargent (2008) provide a textbook treatment of ambiguity aversion and robustness. Hansen and Sargent (2012) clarify several concepts of a robust policymaker and propose a useful nomenclature in terms of three types of ambiguity. ${ }^{2} \mathrm{Kwon}$ and Miao (2017) operationalize the concepts of Hansen and Sargent (2012) in a discrete-time setup.

The papers mentioned in the previous paragraphs put aside the issue of model uncertainty of the private sector. Turning into that, the price manipulation through the endogenous pessimistic expectations of the household in the second part of the paper was first analyzed in Karantounias (2013). In that paper, the policymaker had full confidence in the model, whereas the household was afraid that the model is misspecified. Hansen and Sargent (2012) call this formulation type 0 ambiguity. The current paper, by introducing doubts about the model to both the leader and the follower, nests and generalizes the analysis in Karantounias (2013). Using the ratio of the worst-case beliefs as the relevant indicator of disagreement, it is explicitly shown that the price manipulation through expectation management and the paternalistic incentives can act also in the same direction, and not only in the opposite direction- which was the only outcome when the government had full confidence in the model. Furthermore, a small-doubts approximation is provided, furnishing novel analytical results about the behavior of tax rates and debt. The analysis of the industry problem in the first part of the paper provides also a cautionary tale against jumping to conclusions about expectation management; depending on the specifics of the application, it may be optimal to amplify or mitigate the pessimistic expectations of the follower.

Other relevant contributions are Ferrière and Karantounias (2019), who study distortionary taxation and the design of utility-providing government consumption when there is ambiguity about the business cycle, and Benigno and Paciello (2014), who study the implications of ambiguity aversion of the representative consumer and the policymaker for the design of opti-

\footnotetext{
${ }^{1}$ Other contributions that are motivated by robustness concerns are Orphanides and Williams (2007) and Ajello et al. (2019).

${ }^{2}$ The work of Dennis (2008) is relevant for type I ambiguity. Type II (and type 0) ambiguity is nested in the current paper. Type III ambiguity reflects the approach of Woodford (2010). See further references in Hansen and Sargent (2012).
} 
mal monetary policy. Michelacci and Paciello (2019) associate the credibility of the monetary authority actions to the worst-case beliefs of a heterogenous private sector.

Orlik and Presno (2018) follow the type 0 ambiguity assumptions of Karantounias (2013) and deviate from the commitment assumption by using the notion of sustainable plans. Luo et al. (2014) use a standard ad hoc tax-smoothing model where the policymaker has model uncertainty, and abstract from the general equilibrium determination of the price of government debt.

Other applications of ambiguity aversion outside the realm of optimal policy are Benigno and Nisticò (2012), who study optimal portfolio choice in open economies, Bidder and Smith (2012), who estimate a model of ambiguity aversion with stochastic volatility, and Pouzo and Presno (2016), who generate sovereign ambiguity premia. Croce et al. (2012) analyze positive fiscal policy and ambiguity aversion, and Molavi (2019) constructs a general theory of learning

and misspecification. For another perspective, Christensen (2019) explores identification and estimation of models of robust decision makers.

This paper uses the smooth multiplier preferences of Hansen and Sargent (2001) to analyze optimal policy design. For an example of max-min expected utility see Ilut and Schneider (2014), who analyze the implications of ambiguity aversion for business cycles. Lastly, several new papers interpret survey evidence on expectations through the lens of ambiguity and worst-case beliefs, strengthening the empirical plausibility of ambiguity aversion. Indicatively, Bhandari et al. (2019) look at survey expectations of inflation and unemployment in the United States, Michelacci and Paciello (2020) analyze inflation forecasts at the household level in the United Kingdom, and Bachmann et al. (2020) provide survey evidence on multiple probability models of German manufacturing firms.

\subsection{Organization}

The first part of the paper sets up a dynamic monopolist-competitive fringe Stackelberg game with model uncertainty. Section 2 lays down the environment and section 3 analyzes the management of the pessimistic expectations of the fringe. Similarly, the second part of the paper deals with a classic macroeconomic policy problem. Section 4 describes the economy. Section 5 delves into the fiscal policy problem, contrasts the mitigation of the pessimistic beliefs of the household to the amplification of the fringe's pessimism we found in section 3, and highlights the particular intricacies that paternalism may introduce in a Ramsey problem with model uncertainty. Section 6 concludes. Appendices A and B provide further details on our two environments. A separate Online Appendix provides the details of a small-doubts approximation that may be of independent interest. 


\section{A large firm facing a competitive fringe}

We start the first part of the paper with a dynamic Stackelberg problem with linear-quadratic functional assumptions. This is a classic example in Ljungqvist and Sargent (2004) and Hansen and Sargent (2008), that is used to illustrate the optimal control of models with forward-looking constraints. ${ }^{3}$ Given the linear-quadratic structure, the setup makes also contact with the optimal monetary policy literature.

The setup consists of a large firm with market power, that faces a continuum of small atomistic firms, which are price-takers. For the sake of brevity, we call the large player the 'monopolist', and the (representative) small player the competitive fringe. Both agents produce a single perishable good, whose demand is subject to shocks. Both agents share a reference probability model of demand shocks, which could be misspecified. The reference model, which does not necessarily have to be the true data-generating process, is described by an autoregressive process of order 1 ,

$$
v_{t}=\rho v_{t-1}+C_{\epsilon} \epsilon_{t}
$$

where $C_{\epsilon}>0$ and $v_{-1}=0$. The shock $\epsilon_{t}$ is i.i.d. with zero mean, unitary variance and a reference density $f\left(\epsilon_{t}\right)$. Let $\epsilon^{t} \equiv\left(\epsilon_{0}, \ldots, \epsilon_{t}\right)$ denote the partial history of demand shocks up to $t$. The initial shock $\epsilon_{0}$ (and therefore $v_{0}$ ) is given. The expectation operator $E$ refers to integration with respect to the reference probability model. The inverse demand function for the good is linear and given by

$$
p_{t}\left(\epsilon^{t}\right)=A_{0}-A_{1}\left(Q_{t}+\bar{q}_{t}\right)+v_{t}\left(\epsilon^{t}\right),
$$

where $A_{i}>0, i=0,1 . Q_{t}$ stands for the quantity of the monopolist, and $\bar{q}_{t}$ for the quantity that the competitive fringe produces in equilibrium. It is assumed that the choice of output is made one period ahead, so the relevant quantity variables are pre-determined, that is, they are measurable functions of the history $\epsilon^{t-1}, Q_{t}=Q_{t}\left(\epsilon^{t-1}\right)$ and $\bar{q}_{t}=\bar{q}_{t}\left(\epsilon^{t-1}\right)$.

Monopolist. The cost structure of the monopolist is given by

\footnotetext{
${ }^{3}$ Ljungqvist and Sargent (2004) consider a situation of full confidence in the model by both the monopolist and the competitive fringe (which corresponds to $\sigma_{R}=\sigma_{A}=0$ in our notation). Hansen and Sargent (2008) consider a situation where only the monopolist has doubts about the model $\left(\sigma_{R}<0, \sigma_{A}=0\right)$. Here we allow for both types of agents to exhibit fears of model misspecification, $\left(\sigma_{R}<0, \sigma_{A}<0\right)$.
} 


$$
C_{t}^{M}=e Q_{t}\left(\epsilon^{t-1}\right)+\frac{1}{2} g Q_{t}^{2}\left(\epsilon^{t-1}\right)+\frac{1}{2} c u_{t}^{2}\left(\epsilon^{t}\right)
$$

where $u_{t}\left(\epsilon^{t}\right) \equiv Q_{t+1}\left(\epsilon^{t}\right)-Q_{t}\left(\epsilon^{t-1}\right)$, the change in output produced. Thus, the monopolist faces quadratic adjustment costs, which introduce a dynamic dimension to the profit maximization problem. This feature, together with the fact that $Q_{t+1}$ is predetermined, make output act effectively as capital. The period profits of the monopolist are given by

$$
\Pi_{t}^{M}=p_{t}\left(\epsilon^{t}\right) Q_{t}\left(\epsilon^{t-1}\right)-C_{t}^{M}
$$

Competitive fringe. Turning to the competitive fringe, we let $q_{t}\left(\epsilon^{t-1}\right)$ denote its output choice. We differentiate in notation between $\bar{q}_{t}$ and $q_{t}$ to capture the fact that the fringe is a price-taker, and, as such, it ignores the effect of its production on prices. In equilibrium we have $q_{t}=\bar{q}_{t}$, and this a relationship that the monopolist, as a large player, understands and tries to manipulate. The cost structure of the competitive fringe is similar to the monopolist's,

$$
C_{t}^{F}=d q_{t}\left(\epsilon^{t-1}\right)+\frac{1}{2} h q_{t}^{2}\left(\epsilon^{t-1}\right)+\frac{1}{2} c i_{t}^{2}\left(\epsilon^{t}\right)
$$

where $i_{t}\left(\epsilon^{t}\right) \equiv q_{t+1}\left(\epsilon^{t}\right)-q_{t}\left(\epsilon^{t-1}\right)$, the change in the respective output. The period profits of the competitive fringe are

$$
\Pi_{t}^{F}=p_{t}\left(\epsilon^{t}\right) q_{t}\left(\epsilon^{t-1}\right)-C_{t}^{F}
$$

The cost parameters are positive, $d, h, e, g>0$. We let $c \geq 0$ be non-negative to allow for the possibility of zero adjustment costs $(c=0)$.

\subsection{Competitive fringe and model uncertainty}

Fear of model misspecification. The firm in the competitive fringe has doubts about the reference probability model of demand shocks. Let $f_{t}\left(\epsilon^{t}\right)$ denote the joint density of the history of shocks $\epsilon^{t}$. The firm is afraid that this density is misspecified and considers alternative probability models. We follow Hansen and Sargent (2005) and take the alternative models to be absolutely continuous with respect to the reference model over finite time intervals. This allows us to use the Radon-Nikodym theorem and express these models as a non-negative random variable $M_{t}\left(\epsilon^{t}\right)$, that is a measurable function of $\epsilon^{t}$ with $E M_{t}=1$. We set $M_{0} \equiv 1$, since uncertainty is realized at $t=0 . M_{t}$ has the interpretation of a change of measure and is a martingale with respect to 
the reference model, $E_{t} M_{t+1}=M_{t}$. We can think of $M_{t}$ as an unconditional likelihood ratio, $M_{t}\left(\epsilon^{t}\right)=\frac{f_{t}^{F}\left(\epsilon^{t}\right)}{f_{t}\left(\epsilon^{t}\right)}$ of an alternative density $f_{t}^{F}$ to the reference density, and calculate expectations with respect to the alternative measure as $E^{F}\left(X_{t}\right)=E M_{t} X_{t}$, for a random variable $X_{t}$.

Furthermore, we can decompose $M_{t}$ by defining $m_{t+1}\left(\epsilon^{t+1}\right) \equiv \frac{M_{t+1}\left(\epsilon^{t+1}\right)}{M_{t}\left(\epsilon^{t}\right)}$, which we can think of as the conditional likelihood ratio, i.e. the change in the conditional measure. The conditional likelihood ratio has to integrate to unity, $E_{t} m_{t+1}=1$ in order to be a proper change of measure.

Aversion to model ambiguity. We use the multiplier preferences of Hansen and Sargent (2001), axiomatized by Strzalecki (2011), to express the competitive fringe's aversion to model ambiguity:

$$
\min _{\left\{m_{t+1} \geq 0, M_{t} \geq 0\right\}} E_{0} \sum_{t=0}^{\infty} \beta^{t} M_{t} \Pi_{t}^{F}+\beta \theta_{A} E_{0} \sum_{t=0}^{\infty} \beta^{t} M_{t} \varepsilon_{t}\left(m_{t+1}\right)
$$

subject to $M_{t+1}=m_{t+1} M_{t}$ and $E_{t} m_{t+1}=1$, with $M_{0}=1$ and $0<\theta_{A} \leq \infty$. The criterion (7) uses relative entropy as a measure of discrepancies between distributions,

$$
\varepsilon_{t}\left(m_{t+1}\right) \equiv E_{t} m_{t+1} \ln m_{t+1} .
$$

According to criterion (7), the cautious firm is calculating the present value of profits under alternative probability models, and considers the worst-case scenario. The penalty parameter $\theta_{A}$ penalizes deviations of the alternative model from the reference model in terms of discounted relative entropy, and captures the fear of misspecification of the fringe. The "farther" the alternative model is, the more it is penalized. Full confidence in the model is captured by $\theta_{A}=\infty$. In that case, (7) collapses to a standard present value of profits criterion, which is the case considered by Ljungqvist and Sargent (2004) and Hansen and Sargent (2008).

Problem 1. ('Competitive fringe problem')

The firm in the competitive fringe solves

$$
\max _{\left\{q_{t+1}, u_{t}\right\}} \min _{\left\{m_{t+1} \geq 0, M_{t} \geq 0\right\}} E_{0} \sum_{t=0}^{\infty} \beta^{t} M_{t} \Pi_{t}^{F}+\beta \theta_{A} E_{0} \sum_{t=0}^{\infty} \beta^{t} M_{t} \varepsilon_{t}\left(m_{t+1}\right)
$$

subject to 


$$
\begin{aligned}
q_{t+1} & =q_{t}+i_{t}, t \geq 0 \\
M_{t+1} & =m_{t+1} M_{t}, t \geq 0 \\
E_{t} m_{t+1} & =1, t \geq 0,
\end{aligned}
$$

where $\left(q_{0}, v_{0}\right)$ given and $M_{0} \equiv 1$. The reference model for the demand shock obeys (1) and prices are given by the inverse demand function (2). Period costs and profits are given by (5) and (6) respectively.

Worst-case beliefs. Let asterisks denote the worst-case beliefs of the competitive fringe that solve the minimization problem in criterion (7). We have

$$
m_{t+1}^{*}=\frac{\exp \left(\sigma_{A} V_{t+1}\right)}{E_{t} \exp \left(\sigma_{A} V_{t+1}\right)}, t \geq 0
$$

where $\sigma_{A} \equiv-1 / \theta_{A} \leq 0$ and $V_{t}$ the cautious assessment of the fringe's present value of profits. $V_{t}$ follows the recursion

$$
V_{t}=\Pi_{t}^{F}+\beta\left[E_{t} m_{t+1}^{*} V_{t+1}+\theta_{A} \varepsilon_{t}\left(m_{t+1}^{*}\right)\right]
$$

which, after using (12), takes the familiar risk-sensitive form

$$
V_{t}=\Pi_{t}^{F}+\frac{\beta}{\sigma_{A}} \ln E_{t} \exp \left(\sigma_{A} V_{t+1}\right)
$$

Thus, (12) implies that a cautious competitive fringe that doubts its probability model of demand shocks $\left(\sigma_{A}<0\right)$, assigns high probability (relative to the reference model) on events that have a low present value of profits $V_{t+1}$. Similarly, the fringe is assigning low probability on events that have a high value of profits. Full confidence in the model $\left(\sigma_{A}=0\right)$ leads to $m_{t}^{*}=M_{t}^{*}=1, \forall t$, as expected.

Optimal quantity. The optimal choice of $q_{t+1}$ is governed by the first-order condition

$$
i_{t}=\beta c^{-1} E_{t} m_{t+1}^{*}\left[p_{t+1}-\left(d+h q_{t+1}\right)\right]+\beta E_{t} m_{t+1}^{*} i_{t+1}, t \geq 0 .
$$

Recall that the competitive fringe is facing output adjustment costs. If there were zero adjustment costs $(c=0)$, then (14) would reduce to 


$$
E_{t} m_{t+1}^{*} p_{t+1}=d+h q_{t+1} \equiv M C_{t+1}^{F}
$$

that is, the fringe would equalize its static marginal cost $\left(M C_{t+1}^{F}\right)$ to the expected price under its pessimistic beliefs, as we would expect in a competitive market where output is chosen one period in advance.

Given positive adjustment costs, we can solve (14) forward to get

$$
i_{t}=c^{-1} E_{t} \sum_{j=1}^{\infty} \beta^{j} \frac{M_{t+j}^{*}}{M_{t}^{*}}\left[p_{t+j}-M C_{t+j}^{F}\right]=c^{-1} E_{t}^{F} \sum_{j=1}^{\infty} \beta^{j}\left[p_{t+j}-M C_{t+j}^{F}\right]
$$

where $M_{t}^{*}$ the respective worst-case unconditional likelihood ratio, and $E_{t}^{F}$ shorthand for the conditional expectation operator according to the fringe's worst-case beliefs. Consequently, (16) instructs us that the change in output is proportional to the pessimistic expected present value of all future prices minus the respective marginal costs. High expectations of future prices or low expectations of future marginal costs make the fringe increase more its output for tomorrow versus today.

Pessimistic expectation management? What is crucial to bear in mind is that the monopolist cares about the pessimistic price and marginal costs forecasts of the fringe, because they determine the optimal competitive output choices through (14) or (16). But the quantity produced by the fringe will affect equilibrium prices, and therefore it will affect the profits of the monopolist. This is a standard mechanism that a Stackelberg leader takes into account. In addition to that, the pessimistic beliefs of the competitive fringe are endogenous, since they depend on the present value of profits $V_{t+1}$. This fact is novel in the analysis of the behavior of the fringe and generates for the monopolist an incentive to manipulate the pessimistic expectations by its choice of its future quantities $Q_{t+i}, i \geq 1$. We will see in the next sections that the monopolist will try to amplify the worst-case beliefs of the fringe, in order to reduce the fringe's price forecasts and limit therefore the quantity produced by the fringe.

\subsection{Monopolist and model uncertainty}

Monopolist's alternative models. We use the same machinery as with the competitive fringe in order to express the alternative models that the monopolist is considering. Let $N_{t}\left(\epsilon^{t}\right)$ capture the change of measure of the monopolist, that is $N_{t}$ is a non-negative random variable with $E N_{t}=1 . N_{t}$ is a martingale with respect to the reference model, $E_{t} N_{t+1}=N_{t}$ and can be 
decomposed in terms of conditional likelihood ratios $n_{t+1}$ that integrate to unity, $N_{t+1}=n_{t+1} N_{t}$, with $E_{t} n_{t+1}=1$ and the normalization $N_{0} \equiv 1$.

Evaluation of monopolist's profits. The monopolist's objective is

$$
\min _{\left\{n_{t+1} \geq 0, N_{t} \geq 0\right\}} E_{0} \sum_{t=0}^{\infty} \beta^{t} N_{t} \Pi_{t}^{M}+\beta \theta_{R} E_{0} \sum_{t=0}^{\infty} \beta^{t} N_{t} \varepsilon_{t}\left(n_{t+1}\right)
$$

subject to $N_{t+1}=n_{t+1} N_{t}$ and $E_{t} n_{t+1}=1$, with $N_{0} \equiv 1$ and $0<\theta_{R} \leq \infty$. The penalty parameter $\theta_{R}$ depicts the confidence of the monopolist in the model. Lack of doubts about the model is captured by setting $\theta_{R}=\infty$. $^{4}$

The monopolist acts as a Stackelberg leader that maximizes under commitment profits at $t=0$, subject to the best responses of the competitive fringe, and the inverse demand schedule. The monopolist understands how prices are affected by his output choices and the choices of the fringe in the inverse demand function (2), so he understands that $\bar{q}_{t}=q_{t}$ in equilibrium. By choosing a plan of quantities $\left\{Q_{t+1}\left(\epsilon^{t}\right), u_{t}\left(\epsilon^{t}\right)\right\}$, the monopolist is affecting equilibrium prices, and therefore, profits $V_{t+1}\left(\epsilon^{t+1}\right)$, worst-case beliefs $m_{t+1}^{*}\left(\epsilon^{t+1}\right)$, and quantity choices of the fringe $\left(\bar{q}_{t+1}\left(\epsilon^{t}\right), i_{t}\left(\epsilon^{t}\right)\right)$. The problem of the monopolist takes the following form.

Problem 2. ('Monopolist problem')

The large firm solves

$$
\max _{\left\{Q_{t+1}, u_{t}, \bar{q}_{t+1}, i_{t}, m_{t+1}^{*}, V_{t}\right\}} \min _{\left\{n_{t+1} \geq>0, N_{t} \geq 0\right\}} E_{0} \sum_{t=0}^{\infty} \beta^{t} N_{t} \Pi_{t}^{M}+\beta \theta_{R} E_{0} \sum_{t=0}^{\infty} \beta^{t} N_{t} \varepsilon_{t}\left(n_{t+1}\right)
$$

\footnotetext{
${ }^{4}$ Anticipating the second part of the paper, where a 'Ramsey' policymaker - leader faces as a follower a representative 'agent', we use throughout the paper the subscripts $R$ and $A$ for the penalty parameters of the leader and the follower.
} 
subject to

$$
\begin{aligned}
Q_{t+1} & =Q_{t}+u_{t}, t \geq 0 \\
\bar{q}_{t+1} & =\bar{q}_{t}+i_{t}, t \geq 0 \\
i_{t} & =\beta c^{-1} E_{t} m_{t+1}^{*}\left[p_{t+1}-\left(d+h \bar{q}_{t+1}\right)\right]+\beta E_{t} m_{t+1}^{*} i_{t+1}, t \geq 0 \\
m_{t+1}^{*} & =\frac{\exp \left(\sigma_{A} V_{t+1}\right)}{E_{t} \exp \left(\sigma_{A} V_{t+1}\right)}, t \geq 0 \\
V_{t} & =\Pi_{t}^{F}+\frac{\beta}{\sigma_{A}} \ln E_{t} \exp \left(\sigma_{A} V_{t+1}\right), t \geq 1 \\
N_{t+1} & =n_{t+1} N_{t}, t \geq 0 \\
E_{t} n_{t+1} & =1
\end{aligned}
$$

where $\left(Q_{0}, \bar{q}_{0}, v_{0}\right)$ given and $N_{0} \equiv 1$. The reference model for $v_{t}$ is the process in (1) and the inverse demand function is given by (2). The profits of the monopolist and the fringe are given by (4) and (6) respectively, by setting $q_{t}=\bar{q}_{t}$.

The Stackelberg leader is facing two backward-looking constraints in terms of the predetermined output choices, $Q_{t+1}$ and $\bar{q}_{t+1}$, with laws of motion (18) and (19) respectively. As usual, the monopolist has to take into account the forward-looking constraint that describes the best responses of the fringe, as captured by (20). Nonetheless, the presence of the endogenous pessimistic beliefs $m_{t+1}^{*}$ makes the monopolist take into account the evolution of the profits of the fringe, in order to properly manage the pessimism of the competitive firm. This fact adds one more forward-looking constraint, (22), in comparison to the analysis in Ljungqvist and Sargent (2004) and Hansen and Sargent (2008).

Recursive formulation. Before we proceed to the analysis of the problem, it is useful to make some comments on the recursive formulation of the problem under commitment. Due to the forward-looking constraints, the commitment problem is not recursive in its natural state variables $\left(Q_{t}, \bar{q}_{t}, v_{t}\right)$. We follow Kydland and Prescott (1980) and augment properly the state space in order to achieve such a formulation. We use the change in the fringe's quantity $i_{t}$ and the "expected" profits of the fringe as the two pseudo-state variables that capture the forward-looking constraints (20) and (22). The Appendix provides a detailed description and the respective Bellman equation for the interested reader.

\subsection{Analysis of the Stackelberg leader's problem}

Going back to the monopolist's problem 2, we start with the formation of pessimistic beliefs. 


\subsubsection{Worst-case beliefs of monopolist}

The worst-case beliefs of the monopolist in problem 2 are captured by the conditional likelihood ratio,

$$
n_{t+1}^{*}=\frac{\exp \left(\sigma_{R} W_{t+1}\right)}{E_{t} \exp \left(\sigma_{R} W_{t+1}\right)}, t \geq 0
$$

where $\sigma_{R} \equiv-1 / \theta_{R} \leq 0$. $W_{t}$ stands for the conservative evaluation of the present value of the monopolist's profits. As usual, using the same steps as with the profit recursion of the competitive fringe, we get a risk-sensitive recursion for $W_{t}$,

$$
W_{t}=\Pi_{t}^{M}+\frac{\beta}{\sigma_{R}} \ln E_{t} \exp \left(\sigma_{R} W_{t+1}\right)
$$

Similarly to the competitive fringe in (12), the monopolist assigns high probability on events with a low present value of profits $W_{t+1}$. Lack of doubts $\left(\sigma_{R}=0\right)$ leads to $n_{t}^{*}=N_{t}^{*}=1 \forall t$.

The worst-case beliefs of the monopolist and the competitive fringe may differ for two distinct reasons. First, they may have a different attitude towards model ambiguity, as captured by $\sigma_{R}$ and $\sigma_{A}$. Second, the period profits $\Pi_{t}^{M}$ and $\Pi_{t}^{F}$ differ from each other; so even if $\sigma_{R}=\sigma_{A}$, the worst-case probability assessments can be different.

\subsubsection{Optimal choice of quantities}

Let $\tilde{\xi}_{t}^{i}\left(\epsilon^{t}\right)$ denote the (normalized with the monopolist's likelihood ratio $N_{t}^{*}$ ) multiplier on the forward-looking constraint (20) that involves the change in the fringe's output $i_{t}$. Similarly, let $\tilde{\xi}_{t}^{V}\left(\epsilon^{t}\right)$ denote the (normalized) multiplier on the fringe's profits $V_{t},(22) .^{5}$

Consider the optimal choice of the monopolist output, $Q_{t+1}$. The first-order condition takes the form

$$
\begin{aligned}
c u_{t}= & \beta E_{t} n_{t+1}^{*}[\overbrace{p_{t+1}-A_{1} Q_{t+1}-\left(e+g Q_{t+1}\right)}^{M R_{t+1}^{M}-M C_{t+1}^{M}}]+\beta c E_{t} n_{t+1}^{*} u_{t+1} \\
& -\beta A_{1}[\underbrace{c^{-1} \tilde{\xi}_{t}^{i}}_{\text {indirect effect through fringe's worst-case beliefs }}+\underbrace{\left.\bar{q}_{t+1} E_{t} n_{t+1}^{*} \tilde{\xi}_{t+1}^{V}\right]} .
\end{aligned}
$$

Optimality condition (27) has a straightforward interpretation. To understand the first part in the right-hand side of (27), eliminate the competitive fringe and consider a pure monopoly

\footnotetext{
${ }^{5}$ See the Appendix for the Lagrangian. Throughout the first part of the paper, the "tilde" notation regarding a random variable $X_{t}\left(\epsilon^{t}\right)$ refers to a normalization by the monopolist's worst-case likelihood ratio $N_{t}^{*}, \tilde{X}_{t} \equiv \frac{X_{t}}{N_{t}^{*}}$.
} 
(so that $\tilde{\xi}_{t}^{i}=\tilde{\xi}_{t}^{V}=0$ in $(27)$ ). Then, if there were no adjustment costs $(c=0)$, the monopolist would produce so much so that the expected (under its worst-case scenario) marginal revenue $\left(M R_{t+1}^{M} \equiv p_{t+1}-A_{1} Q_{t+1}\right)$ would equal the expected static marginal cost $\left(M C_{t+1}^{M} \equiv e+g Q_{t+1}\right)$. The monopolist would deviate partially from this rule, to the extent there were some adjustment $\operatorname{costs} c>0$ and increase or decrease output according to the entire present value of differences between future marginal revenues and costs, similarly to the dynamic choice of output by the fringe in (16).

Turning to a situation with a competitive fringe, the 'monopolist' acts as a Stackelberg leader. Thus, he takes into account that an increase in quantity reduces prices and therefore it reduces the output produced by the fringe, as captured by (14) (or (15) when there are no adjustment costs). The shadow value to the monopolist of this reduction of price is given by $\tilde{\xi}_{t}^{i}$, which multiplies the third term in the right-hand side of (27). But when beliefs are endogenous, the monopolist has also to takes into account how an increase in $Q_{t+1}$ affects them. A fall in prices through an increase of monopoly output, reduces the profits of the fringe, for each realization of the shock next period (recall that $Q_{t+1}$ is pre-determined). This reduction in profits alters the worst-case beliefs of the fringe, and bears an average shadow value of $E_{t} n_{t+1}^{*} \tilde{\xi}_{t+1}^{V}$, multiplied with the quantity that the fringe produces, $\bar{q}_{t+1}$. This is the fourth term in the right-hand side of $(27)$.

Consider now the optimal choice of the fringe's output. Let $\tilde{\lambda}_{t}^{\bar{q}}$ denote the normalized multiplier on the law of motion of the fringe's output (19). The optimality condition with respect to $\bar{q}_{t+1}$ is given by

$$
\begin{aligned}
\tilde{\lambda}_{t}^{\bar{q}}= & \underbrace{-\beta A_{1} Q_{t+1}}_{\text {reduction in monopolist's profits }}+\beta E_{t} n_{t+1}^{*} \tilde{\lambda}_{t+1}^{\bar{q}}-\underbrace{\beta c^{-1}\left(A_{1}+h\right) \tilde{\xi}_{t}^{i}}_{\text {reduction in } p-M C^{F}} \\
& +\beta \underbrace{E_{t} n_{t+1}^{*} \tilde{\xi}_{t+1}^{V}}_{\text {belief manipulation }} \overbrace{\left[R_{t+1}-A_{1} \bar{q}_{t+1}-\left(d+h \bar{q}_{t+1}\right)\right]}^{M C_{t+1}^{F}}
\end{aligned}
$$

Condition (28) has a similar interpretation. An increase in $\bar{q}_{t+1}$ reduces the profits of the monopolist through a reduction in prices (first term in the right-hand side of (28)). Furthermore, this reduction in prices and the accompanied increase in marginal costs reduces the difference between prices and marginal costs in the best response of the fringe, bearing shadow value of $\tilde{\xi}_{t}^{i}$ (third term in the right-hand side of (28)). As before, a change in output $\bar{q}_{t+1}$ affects the fringe's marginal profits at $t+1$, captured by the difference in the marginal revenue and marginal cost $\left(M R^{F}-M C^{F}\right)$, and therefore, its worst-case beliefs, with an average shadow value measured by the last term in (28). 


\subsubsection{Law of motion of $\tilde{\xi}_{t}^{i}$}

Being the (normalized) multiplier on the best response of the competitive fringe (20), $\tilde{\xi}_{t}^{i}$ captures the shadow value to the Stackelberg leader of the fringe's pessimistic evaluation of the present discounted value of differences between future prices and marginal costs, as seen in (16). The first-order condition with respect to $i_{t}$ is

$$
\tilde{\xi}_{t}^{i}=\tilde{\lambda}_{t}^{\bar{q}} \underbrace{-c i_{t} \tilde{\xi}_{t}^{V}}_{\text {endogenous beliefs effect }}+\frac{m_{t}^{*}}{n_{t}^{*}} \underbrace{\tilde{\xi}_{t-1}^{i}}_{\text {old promises }}
$$

with $\tilde{\xi}_{-1}^{i} \equiv 0$. As usual, the initial value of the multiplier is zero, since at $t=0$ there are no output promises to be kept by the monopolist.

To interpret the condition, consider first the case where neither the monopolist nor the fringe have fear of misspecification $\left(\sigma_{R}=\sigma_{A}=0\right)$, so that $n_{t}^{*}=m_{t}^{*}=1$. Note also that if the fringe has full confidence in the model, then the monopolist has no reason to keep track of the fringe's profits, so $\tilde{\xi}_{t}^{V}=0$. This is the prominent textbook case of Ljungqvist and Sargent (2004). Then, (29) simplifies to

$$
\tilde{\xi}_{t}^{i}=\tilde{\lambda}_{t}^{\bar{q}}+\tilde{\xi}_{t-1}^{i}
$$

and, therefore, the shadow value of $i_{t}$ would just be the cumulative sum of all multipliers

$\lambda_{t}^{\bar{q}}, \tilde{\xi}_{t}^{i}=\sum_{j=0}^{t} \tilde{\lambda}_{j}^{\bar{q}}$, a result of the commitment of the monopolist to the promises induced by the forward-looking constraint (20). In other words, exactly because the monopolist commits to his plan at $t=0$, he takes into account that a change of the fringe's output at time $t$ (which has shadow value $\tilde{\lambda}_{t}^{\bar{q}}$ ) will affect all the price forecasts of a forward-looking competitive fringe up to time $t$. Turning now model uncertainty on $\left(\sigma_{R}<0, \sigma_{A}<0\right)$, in addition to the standard commitment to old promises that we analyzed, the monopolist has also to take into account that an increase in $i_{t}$ reduces the profits of the fringe and, therefore, it affects its worst-case beliefs. This effect is captured by the term $c i_{t} \tilde{\xi}_{t}^{V}$ in (29).

What if only the monopolist has doubts about the model $\left(\sigma_{R}<0, \sigma_{A}=0\right)$ ? Hansen and Sargent (2012) call this case type II ambiguity. The law of motion of $\tilde{\xi}_{t}^{i}$ would take the form

$$
\tilde{\xi}_{t}^{i}=\tilde{\lambda}_{t}^{\bar{q}}+\frac{1}{n_{t}^{*}} \tilde{\xi}_{t-1}^{i}, \tilde{\xi}_{-1}^{i} \equiv 0
$$

In that case, even if the multiplier $\tilde{\xi}_{t}^{V}$ on the profits of the fringe is vanishing, there is still a non-linearity in the law of motion through the conditional likelihood ratio $n_{t}^{*}$ of the monopolist. 
This is the reason why there are no closed-form solutions even in the linear-quadratic case. ${ }^{6}$

\section{Managing the pessimistic expectations of the fringe}

Of central importance is $\tilde{\xi}_{t}^{V}$, the multiplier that captures the shadow value (to the monopolist) of the fringe's profits, to the extent they affect the pessimistic forecasts of future prices. As such, it encapsulates how the monopolist manages the worst-case beliefs of the competitive firm.

The first-order condition with respect to the profits of the fringe $V_{t}$ delivers

$$
\tilde{\xi}_{t}^{V}=\sigma_{A} \frac{m_{t}^{*}}{n_{t}^{*}} \underbrace{\left[\mu_{t}-E_{t-1} m_{t}^{*} \mu_{t}\right]}_{\text {net value of reducing } m_{t}^{*}} \frac{1}{N_{t-1}^{*}}+\frac{m_{t}^{*}}{n_{t}^{*}} \underbrace{\tilde{\xi}_{t-1}^{V}}_{\text {old promises }}, t \geq 1,
$$

where $\mu_{t}$ the multiplier on the worst-case conditional likelihood ratio of the fringe, (21). As expected, when $\sigma_{A}=0$ we have $\tilde{\xi}_{t}^{V}=0$. Furthermore, since uncertainty is realized at $t=0$, the shadow value of profits at $t=0$ is zero, $\tilde{\xi}_{0}^{V} \equiv 0$. Is it easy to see from (32) that $\tilde{\xi}_{t}^{V}$ generates persistence to the optimal choices of the Stackelberg leader: $\tilde{\xi}_{t}^{V}$ is a martingale with respect to the worst-case beliefs of the monopolist, $E_{t-1} n_{t}^{*} \tilde{\xi}_{t}^{V}=\tilde{\xi}_{t-1}^{V}$, since $E_{t-1} m_{t}^{*}\left(\mu_{t}-E_{t-1} m_{t}^{*} \mu_{t}\right)=0$. Hence, the average value of $\tilde{\xi}_{t}^{V}$ is zero, $E N_{t}^{*} \tilde{\xi}_{t}^{V}=0$, and it can take both positive and negative values. ${ }^{7}$

How does the monopolist manage the pessimistic expectations of the fringe? Consider an increase in the profits of the fringe $V_{t}$. An increase in profits makes the pessimistic fringe assign a lower conditional probability mass on $\epsilon_{t}$, leading to a reduction in $m_{t}^{*}\left(\sigma_{A}<0\right)$. The benefit or cost of a reduction of $m_{t}^{*}$ is captured by the shadow value of $m_{t}^{*}$, which is just the multiplier $\mu_{t}$. Moreover, a reduction in the conditional probability of $\epsilon_{t}$ leads necessarily to an increase in the probabilities of the other nodes $\hat{\epsilon}_{t} \neq \epsilon_{t}$ of the event tree, so that the total probability mass is unity. This increase in the rest of the nodes has shadow value of $E_{t-1} m_{t}^{*} \mu_{t}$, so the net effect of increasing $V_{t}$ is captured by $\sigma_{A}\left(\mu_{t}-E_{t-1} m_{t}^{*} \mu_{t}\right)$ in (32). Note that a change in current profits $V_{t}$ affects the fringe's evaluation of the present value of profits in the periods preceding $t$ (and thus its worst-case scenarios the previous periods). Thus, as in the analysis of the law of motion of $\tilde{\xi}_{t}^{i}$ in $(29)$, the commitment of the monopolist makes him to take into account the shadow value of its old promises, $\tilde{\xi}_{t-1}^{V}$, in (32). In other words, $\tilde{\xi}_{t}^{V}$ cumulates the shadow value of changing all the conditional likelihood ratios up to period $t$.

\footnotetext{
${ }^{6}$ This is so even if we make additional Gaussian assumptions on the reference model of the shock $v_{t}$. Recall that in the absence of model-uncertainty $\left(\sigma_{A}=\sigma_{R}=0\right)$ we have closed-form solutions, see for example Ljungqvist and Sargent (2004). See Kwon and Miao (2017) for a general analysis of the three types of ambiguity of Hansen and Sargent (2012) in a discrete-time setup.
}

${ }^{7}$ We can use the martingale result to replace $E_{t} n_{t+1}^{*} \tilde{\xi}_{t+1}^{V}$ with $\tilde{\xi}_{t}^{V}$ in (27). 
What is the shadow value of the likelihood ratio $m_{t}^{*}$ to the monopolist? The first-order condition with respect to $m_{t}^{*}$ is

$$
\mu_{t}=\left[c^{-1} C_{\epsilon} \epsilon_{t}+i_{t}\right] \tilde{\xi}_{t-1}^{i} N_{t-1}^{*}, t \geq 1
$$

Condition (33) is intuitive. An increase in $m_{t}^{*}$ at a particular shock means that the fringe is putting higher conditional probability at $\epsilon_{t}$. The benefit to the monopolist $\mu_{t}$ should be associated with the size of $\epsilon_{t}$ (that has now higher probability mass) and the expected difference of future prices and marginal costs, which determines the fringe's best response $i_{t}$, as seen from (16). The induced change of competitive output due to changes in the probability mass has shadow value $\tilde{\xi}_{t-1}^{i}$ to the monopolist.

Using (33), the law of motion of $\tilde{\xi}_{t}^{V}$ finally becomes

$$
\tilde{\xi}_{t}^{V}=\sigma_{A} \frac{m_{t}^{*}}{n_{t}^{*}}[c^{-1} \underbrace{C_{\epsilon}\left(\epsilon_{t}-E_{t-1} m_{t}^{*} \epsilon_{t}\right)}_{p_{t}-E_{t-1} m_{t}^{*} p_{t}}+\left(i_{t}-E_{t-1} m_{t}^{*} i_{t}\right)] \tilde{\xi}_{t-1}^{i}+\frac{m_{t}^{*}}{n_{t}^{*}} \tilde{\xi}_{t-1}^{V}, \tilde{\xi}_{0}^{V} \equiv 0 .
$$

Define now

$$
I_{t} \equiv c^{-1}\left[p_{t}-M C_{t}^{F}\right]+i_{t}=c^{-1} E_{t} \sum_{j=0}^{\infty} \beta^{j} \frac{M_{t+j}^{*}}{M_{t}^{*}}\left[p_{t+j}-M C_{t+j}^{F}\right]
$$

$I_{t}$ is the sum of the current difference of prices and marginal costs and the present value of future differences in prices and marginal costs, $i_{t}$, as seen from (16). Thus, the "surprise" in $I_{t}$ is

$$
I_{t}-E_{t-1} m_{t}^{*} I_{t}=c^{-1} C_{\epsilon}\left(\epsilon_{t}-E_{t-1} m_{t}^{*} \epsilon_{t}\right)+\left(i_{t}-E_{t-1} m_{t}^{*} i_{t}\right) .
$$

Consequently, we can rewrite (34) as

$$
\tilde{\xi}_{t}^{V}=\sigma_{A} \frac{m_{t}^{*}}{n_{t}^{*}}\left[I_{t}-E_{t-1} m_{t}^{*} I_{t}\right] \tilde{\xi}_{t-1}^{i}+\frac{m_{t}^{*}}{n_{t}^{*}} \tilde{\xi}_{t-1}^{V}, \tilde{\xi}_{0}^{V} \equiv 0
$$

Therefore, the increment to the martingale $\tilde{\xi}_{t}^{V}$ depends on the "news" (under the fringe's worst-case measure) in $I_{t}$. The news can be further decomposed as "news" in the present value of prices and "news" in the present value of marginal costs, 


$$
I_{t}-E_{t-1} m_{t}^{*} I_{t}=c^{-1}[\underbrace{\left(E_{t}^{F}-E_{t-1}^{F}\right) \sum_{i=0}^{\infty} \beta^{i} p_{t+i}}_{\text {news in prices }}-\underbrace{\left(E_{t}^{F}-E_{t-1}^{F}\right) \sum_{i=0}^{\infty} \beta^{i} M C_{t+i}^{F}}_{\text {news in marginal costs }}] .
$$

In order to dig deeper in the analysis, we need to understand the sign of $\tilde{\xi}_{t}^{i}$, which gives us information on how the monopolist wants to affect the quantity choice of the fringe. We consider in the next section a situation with zero adjustment costs, that allows us to simplify the "news" terms in (38) and to sign $\tilde{\xi}_{t}^{i}$.

\subsection{Zero adjustment costs}

Let $c=0$. The lack of adjustment costs allows us to discard $\left(Q_{t}, \bar{q}_{t}\right)$ as state variables, and simplifies the problem by making only the one-period ahead price forecast matter.

In particular, recall from (15) that when there are no adjustment costs, the fringe is setting its marginal cost equal to the worst-case expectation of future prices, $E_{t} m_{t+1}^{*} p_{t+1}=d+h \bar{q}_{t+1}$. By using the inverse demand function (2), we can rewrite the optimality condition of the fringe in terms of the equilibrium competitive quantity,

$$
\bar{q}_{t+1}=\frac{A_{0}-d-A_{1} Q_{t+1}+\rho v_{t}+C_{\epsilon} E_{t} m_{t+1}^{*} \epsilon_{t+1}}{A_{1}+h} .
$$

The equilibrium reaction of the competitive fringe in (39) illuminates a direct and an indirect effect of the monopolist's actions on the quantity that the competitive fringe is producing. The direct effect is the typical Stackelberg effect: an increase in $Q_{t+1}$ reduces expected prices and decreases therefore the quantity that the fringe is producing. The indirect effect comes from the pessimistic forecasts of demand shocks, $E_{t} m_{t+1}^{*} \epsilon_{t+1}$. An increase in expected demand leads to an increase in the quantity that the fringe will produce according to (39). Since the fringe's expectations are endogenous, it is natural to conjecture that the monopolist has an incentive to make the fringe more pessimistic; a low forecast of the demand shock makes the fringe produce less, reducing its share in the market and allowing therefore the monopolist to increase its quantity and profits. We will see that this is exactly the mechanism that emerges.

Proposition 1. ('Zero adjustment costs')

The monopolist's problem for $c=0$ is to choose $\left\{Q_{t+1}, \bar{q}_{t+1}, m_{t+1}^{*}, V_{t}\right\}$ to maximize the worstcase present value of profits (17) subject to the reaction function of the fringe, (15), the worst-case beliefs of the fringe and its profit recursion (for $c=0$ ), (21) and (22), the reference process for the demand shock (1) and the inverse demand function (2), given initial values $\left(Q_{0}, \bar{q}_{0}, v_{0}\right)$. 
- The optimality conditions with respect to $(Q, q)$ are

$$
\begin{array}{cc}
Q_{t+1}: & E_{t} n_{t+1}^{*}\left[p_{t+1}-A_{1} Q_{t+1}-\left(e+g Q_{t+1}\right)\right]-A_{1}\left[\tilde{\xi}_{t}^{i}+\tilde{\xi}_{t}^{V} \bar{q}_{t+1}\right]=0 \\
\bar{q}_{t+1}: & -A_{1} Q_{t+1}-\left(A_{1}+h\right) \tilde{\xi}_{t}^{i}+E_{t} n_{t+1}^{*} \tilde{\xi}_{t+1}^{V}\left[p_{t+1}-A_{1} \bar{q}_{t+1}-\left(d+h \bar{q}_{t+1}\right)\right]=0
\end{array}
$$

The worst-case likelihood ratio of the monopolist is determined by (25) and its profits by the respective risk-sensitive recursion (26), for the case of zero adjustment costs.

- The shadow value of the conditional likelihood ratio $m_{t}^{*}$ is

$$
\mu_{t}=C_{\epsilon} \epsilon_{t} \tilde{\xi}_{t-1}^{i} N_{t-1}^{*}
$$

Hence, the law of motion of the martingale $\tilde{\xi}_{t}^{V}$ simplifies to

$$
\tilde{\xi}_{t}^{V}=\sigma_{A} \frac{m_{t}^{*}}{n_{t}^{*}} C_{\epsilon}\left(\epsilon_{t}-E_{t-1} m_{t}^{*} \epsilon_{t}\right) \tilde{\xi}_{t-1}^{i}+\frac{m_{t}^{*}}{n_{t}^{*}} \tilde{\xi}_{t-1}^{V}, \tilde{\xi}_{0}^{V} \equiv 0
$$

- When $c=0$, the multiplier $\tilde{\xi}_{t}^{i}$ stands for the shadow value (to the monopolist) of the fringe's price forecast. Using (41) and (43) we can show that

$$
\tilde{\xi}_{t}^{i}=-\frac{A_{1}}{A_{1}+h-\sigma_{A} C_{\epsilon}^{2} \operatorname{Var}_{t}^{F}\left(\epsilon_{t+1}\right)}\left[Q_{t+1}+\tilde{\xi}_{t}^{V} \bar{q}_{t+1}\right], t \geq 0
$$

where $\operatorname{Var}_{t}^{F}($.$) stands for the conditional variance according to the worst-case measure of$ the fringe, $\operatorname{Var}_{t}^{F}\left(\epsilon_{t+1}\right) \equiv E_{t} m_{t+1}^{*}\left(\epsilon_{t+1}-E_{t} m_{t+1}^{*}\right)^{2}$.

Proof. See the Appendix for the Lagrangian and the derivations. When $c=0$, the dynamics of $\tilde{\xi}_{t}^{i}$ are muted (recall the law of motion (29)), allowing us to focus on the dynamics of $\tilde{\xi}_{t}^{V}$.

\subsubsection{Build intuition: $\sigma_{A}=0$}

In order to build intuition, assume that the competitive fringe has no doubts about the model $\left(\sigma_{A}=0\right)$, so that there is no belief management and $\tilde{\xi}_{t}^{V}=0 .{ }^{8}$ In that case we have from (44)

\footnotetext{
${ }^{8}$ When $c=0$ and $\sigma_{A}=0$, the problem of the robust monopolist becomes a standard problem without forwardlooking constraints, since then the only expectation in the behavior of the fringe is exogenous and actually zero, $E_{t} \epsilon_{t+1}=0$.
} 


$$
\tilde{\xi}_{t}^{i}=-\frac{A_{1}}{A_{1}+h} Q_{t+1}<0
$$

The fact that the multiplier is negative means that the monopolist has an incentive to reduce the price forecast of the follower. This captures the shadow value of the direct effect that we highlighted earlier. The reason is simple: a decrease in prices leads to a reduction in quantity produced by the follower and therefore larger profits for the monopolist. The first-order condition of the cautious monopolist (40) becomes

$$
E_{t} n_{t+1}^{*}\left[p_{t+1}-A_{1} Q_{t+1}-\left(e+g Q_{t+1}\right)\right]-A_{1} \tilde{\xi}_{t}^{i}=0
$$

As usual, the first term in the left-hand side denotes the expected marginal profit that the monopolist is getting with an increase in quantity $Q_{t+1}$. The second term in the lefthand side denotes the Stackelberg benefit (since $\tilde{\xi}_{t}^{i}<0$ ) of increasing quantity. An increase in quantity decreases prices and therefore the quantity produced by the follower. This mitigates the reduction in prices than a given increase in $Q_{t+1}$ has.

\subsubsection{Amplifying worst-case beliefs}

The sign of $\tilde{\xi}_{t}^{i}$ is important because it reveals whether the monopolist wants the fringe to increase or decrease its price forecast. Going back to the case of $\sigma_{A}<0$, we see from (44) that $\tilde{\xi}_{t}^{i}<0$ as long as $\tilde{\xi}_{t}^{V}$ is not "too" negative, $\tilde{\xi}_{t}^{V}>-Q_{t+1} / \bar{q}_{t+1}$. Recall that the multiplier $\tilde{\xi}_{t}^{V}$ takes both positive and negative values with average value zero. The lower bound for a negative $\tilde{\xi}_{t}^{V}$ is not too stringent as long as the ratio $Q_{t+1} / \bar{q}_{t+1}$ is large enough, that is, as long as the monopolist quantity is large relative to the competitive fringe quantity. Assume for the rest of the section that $\tilde{\xi}_{t}^{V}>-Q_{t+1} / \bar{q}_{t+1}$, so that the monopolist benefits if the pessimistic price forecasts of the fringe are reduced $\left(\tilde{\xi}_{t}^{i}<0\right)$.

High and low demand shocks. What is the net effect of increasing $m_{t}^{*}$, as captured by $\mu_{t}-E_{t-1} m_{t}^{*} \mu_{t}$ ? Using (42), we see that $\mu_{t}-E_{t-1} m_{t}^{*} \mu_{t}$ is negative in good times, i.e. when demand shocks are above average, $\epsilon_{t}>E_{t-1} m_{t}^{*} \epsilon_{t}$, and $\mu_{t}-E_{t-1} m_{t}^{*} \mu_{t}$ is positive in bad times, i.e. when demand shocks are below average, $\epsilon_{t}<E_{t-1} m_{t}^{*} \epsilon_{t} \cdot{ }^{9}$ Hence, the monopolist wants to decrease the probability mass that the pessimistic fringe is assigning on good times and he wants to increase the probability mass that the fringe is assigning on bad times. But the pessimistic fringe is either way assigning low probability on good times, where profits are high, and higher

\footnotetext{
${ }^{9}$ When we have positive adjustment costs, "good" times correspond to positive news in $I_{t}($ see $(36)$ and (38)), and "bad" times to negative news in $I_{t}$.
} 
probability on bad times, since profits are low. Thus, the monopolist is trying to amplify the pessimistic beliefs of the competitive fringe. And the intuition is simple, as illuminated by (39). A lower forecast of demand shocks leads to a smaller quantity by the fringe, allowing the monopolist to have higher profits.

The monopolist twists the pessimistic beliefs of the competitive fringe by affecting the fringe's present value of profits $V_{t}$, which have shadow value $\tilde{\xi}_{t}^{V}$. We summarize the implications of the law of motion (43) in terms of a proposition.

Proposition 2. ('Good versus bad times') Assume without loss of generality that $\epsilon_{t}$ takes two values $\epsilon_{H}>0>\epsilon_{L}$ with mean value zero under the reference model. Let $x_{t}(i), i=H, L$ denote the value of a variable when $\epsilon_{t}=\epsilon_{i}, i=H, L$.

a) Assume that $\tilde{\xi}_{t-1}^{V}$ is at its average value, which is zero, $\tilde{\xi}_{t-1}^{V}=0$. Then $\tilde{\xi}_{t}^{V}(H)>0>\tilde{\xi}_{t}^{V}(L)$.

b) Assume that $\tilde{\xi}_{t-1}^{V}>-Q_{t} / \bar{q}_{t}$ and that $\left(\sigma_{R}, \sigma_{A}\right)$ and the monopolist's and fringe's profits are such so that $m_{t}^{*}(H)=n_{t}^{*}(H)$. Then, $\tilde{\xi}_{t}^{V}(H)>\tilde{\xi}_{t-1}^{V}>\tilde{\xi}_{t}^{V}(L)$.

Proof. We obviously have $\epsilon_{H}>E_{t-1} m_{t}^{*} \epsilon_{t}>\epsilon_{L}$. a) When $\tilde{\xi}_{t-1}^{V}=0$, we get from (44) that $\tilde{\xi}_{t-1}^{i}<0$. Thus, from (43) we get $\xi_{t}(H)=\sigma_{A} \frac{m_{t}^{*}(H)}{n_{t}^{*}(H)} C_{\epsilon}\left[\epsilon_{H}-E_{t-1} m_{t}^{*} \epsilon_{t}\right] \tilde{\xi}_{t-1}^{i}>0>\tilde{\xi}_{t}^{V}(L)=$ $\sigma_{A} \frac{m_{t}^{*}(L)}{n_{t}^{*}(L)} C_{\epsilon}\left[\epsilon_{L}-E_{t-1} m_{t}^{*} \epsilon_{t}\right] \tilde{\xi}_{t-1}^{i}$. b) (44) implies again that $\tilde{\xi}_{t-1}^{i}<0$. Furthermore, since the conditional likelihood ratios have to add to unity according to the reference measure, we have $m_{t}^{*}(L)=n_{t}^{*}(L)$. So the conditional belief ratios $m_{t}^{*} / n_{t}^{*}$ in (43) become identically unity and the result follows.

The proposition says that, starting from the unconditional average, the shadow value of the fringe's profits is positive in good times and negative in bad times. Or, more generally, if there is not a lot of disagreement in the probabilistic assessment of contingencies between the monopolist and the fringe, the shadow value of the fringe's profits increases in good times and decreases in bad times. This behavior of the multiplier has again to do with how the monopolist amplifies the worst-case beliefs of the fringe. By increasing the profits of the fringe in good times and decreasing the profits of the fringe in bad times, the competitive fringe tilts even more its pessimistic assessments towards low demand shocks.

The instrument of the monopolist is its quantity, $Q_{t+1}$, which is pre-determined, as we have emphasized, $Q_{t+1}\left(\epsilon^{t}\right)$. How does it change when the multiplier $\tilde{\xi}_{t}^{V}$ increases (as in good times)? Proposition 3 gives a partial answer.

Proposition 3. Keep the conditional worst-case moments $E_{t} m_{t+1}^{*} \epsilon_{t+1}, E_{t} n_{t+1}^{*} \epsilon_{t+1}$ and $\operatorname{Var}_{t}^{F}\left(\epsilon_{t+1}\right)$ constant. Then: 
- An increase in $\tilde{\xi}_{t}^{V}$ decreases the monopolist's quantity at $t+1$ and increases the fringe's quantity,

$$
\begin{aligned}
& \left.\frac{\partial Q_{t+1}}{\partial \tilde{\xi}_{t}^{V}}\right|_{\text {moments const. }}<0 \\
& \left.\frac{\partial \bar{q}_{t+1}}{\partial \tilde{\xi}_{t}^{V}}\right|_{\text {moments const. }}>0 .
\end{aligned}
$$

- An increase in $\tilde{\xi}_{t}^{V}$ reduces the total quantity produced for $t+1, Q_{t+1}^{\text {total }} \equiv Q_{t+1}+\bar{q}_{t+1}$,

$$
\left.\frac{\partial Q_{t+1}^{\text {total }}}{\partial \tilde{\xi}_{t}^{V}}\right|_{\text {moments const. }}<0
$$

Proof. In the Appendix we show how, given the conditional moments of the monopolist and the fringe at time $t$, we can solve for $\left(Q_{t+1}, \bar{q}_{t+1}\right)$ as a (non-linear) function of $\left(\tilde{\xi}_{t}^{V}, v_{t}\right)$. We perform then a ceteris-paribus exercise.

By choosing $Q_{t+1}\left(\epsilon^{t}\right)$ for next period, the monopolist affects the current present value of the fringe's profits, $V_{t}\left(\epsilon^{t}\right)$ and therefore the current likelihood ratio $m_{t}^{*}$. We expect that when $\tilde{\xi}_{t}^{V}$ increases, the monopolist has an incentive to increase the fringe's current profits in order to induce the fringe to reduce the current likelihood ratio. And the monopolist can achieve that by reducing $Q_{t+1}$. This decrease in the monopolist's quantity increases the expectation of future competitive fringe profits, increasing therefore current profits $V_{t}$. And this reduction in quantity induces also the fringe to produce more for next period (but not as much as to increase the total quantity in the market). Proposition 3 confirms partially this intuition. Taking into account also proposition 2, which shows how $\tilde{\xi}_{t}^{V}$ varies in good and bad times, we expect the monopolist to decrease its quantity for next period in good times, and increase its quantity for next period in bad times. The next section provides a simple illustration.

\subsubsection{Illustration}

Consider now a three-period example with zero adjustment costs. At $t=0$ and at $t=2$ there is no uncertainty and the demand shock is zero, $v_{0}=v_{2}=0$. At $t=1$ the demand shock is $v_{1}=C_{\epsilon} \epsilon_{1}$. The shock $\epsilon_{1}$ has a reference distribution with zero mean and unitary variance. The monopolist and the competitive fringe form at $t=0$ a worst-case distribution of $\epsilon_{1}$, captured by the changes of measure $n_{1}^{*}\left(\epsilon_{1}\right)$ and $m_{1}^{*}\left(\epsilon_{1}\right)$ respectively. The initial quantities $\left(Q_{0}, \bar{q}_{0}\right)$ are given. 

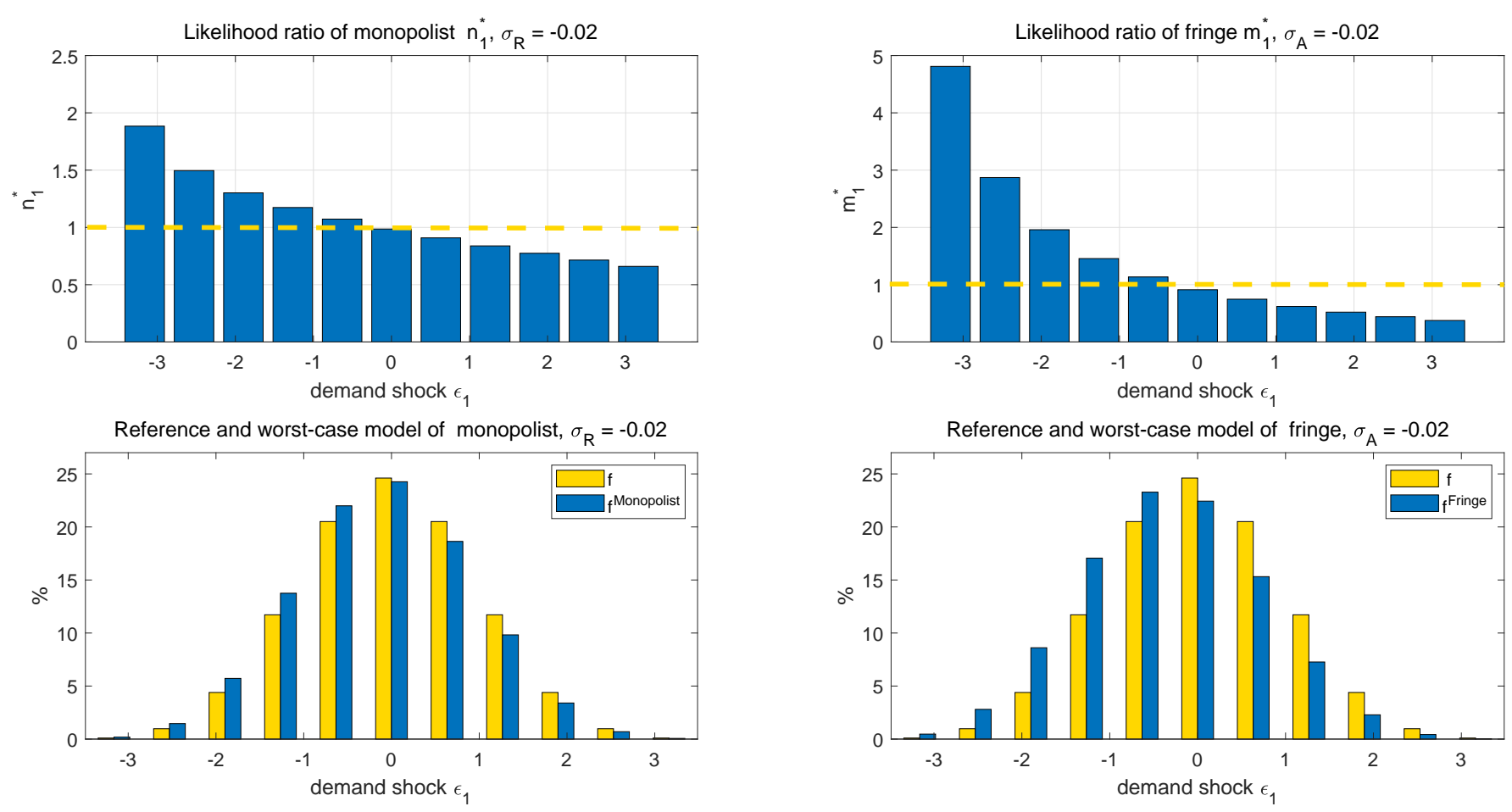

Figure 1: The top panels display the worst-case likelihood ratios of the monopolist and the fringe (the dotted line depicts the full-confidence case, $n_{1}^{*}=m_{1}^{*}=1$ ). The bottom panels contrast the respective worst-case distributions to the reference distribution $f$, which has zero mean and unitary variance. See the Appendix for the parametrization of the example.

Given the timing assumptions, $\left(Q_{1}, \bar{q}_{1}\right)$ are not random. Quantities at $t=2$ are functions of $\epsilon_{1}$, $\left(Q_{2}\left(\epsilon_{1}\right), \bar{q}_{2}\left(\epsilon_{1}\right)\right)$. The fringe's behavior at $t=1$ and at $t=2$ is given by

$$
\begin{aligned}
E m_{1}^{*} p_{1} & =d+h \bar{q}_{1} \\
p_{2}\left(\epsilon_{1}\right) & =d+h \bar{q}_{2}\left(\epsilon_{1}\right), \forall \epsilon_{1} .
\end{aligned}
$$

Similarly, the manipulation of the fringe's beliefs is captured by the multiplier $\tilde{\xi}_{1}^{V}\left(\epsilon_{1}\right)$, that takes positive and negative values in good and bad times respectively, signaling that the monopolist wants to increase the profits of the fringe in good times, and decrease them in bad times. The Appendix provides the details of the exercise.

Worst-case beliefs. Figure 1 displays the worst-case conditional likelihood ratios and the resulting worst-case distributions for the monopolist and the competitive fringe for $\sigma_{R}=\sigma_{A}=$ -0.02 . As expected, the pessimistic monopolist and the competitive fringe assign higher probability mass on low demand shocks. Note that the competitive fringe is putting relatively more mass than the monopolist on bad demand shocks, despite sharing the same value of the penalty 

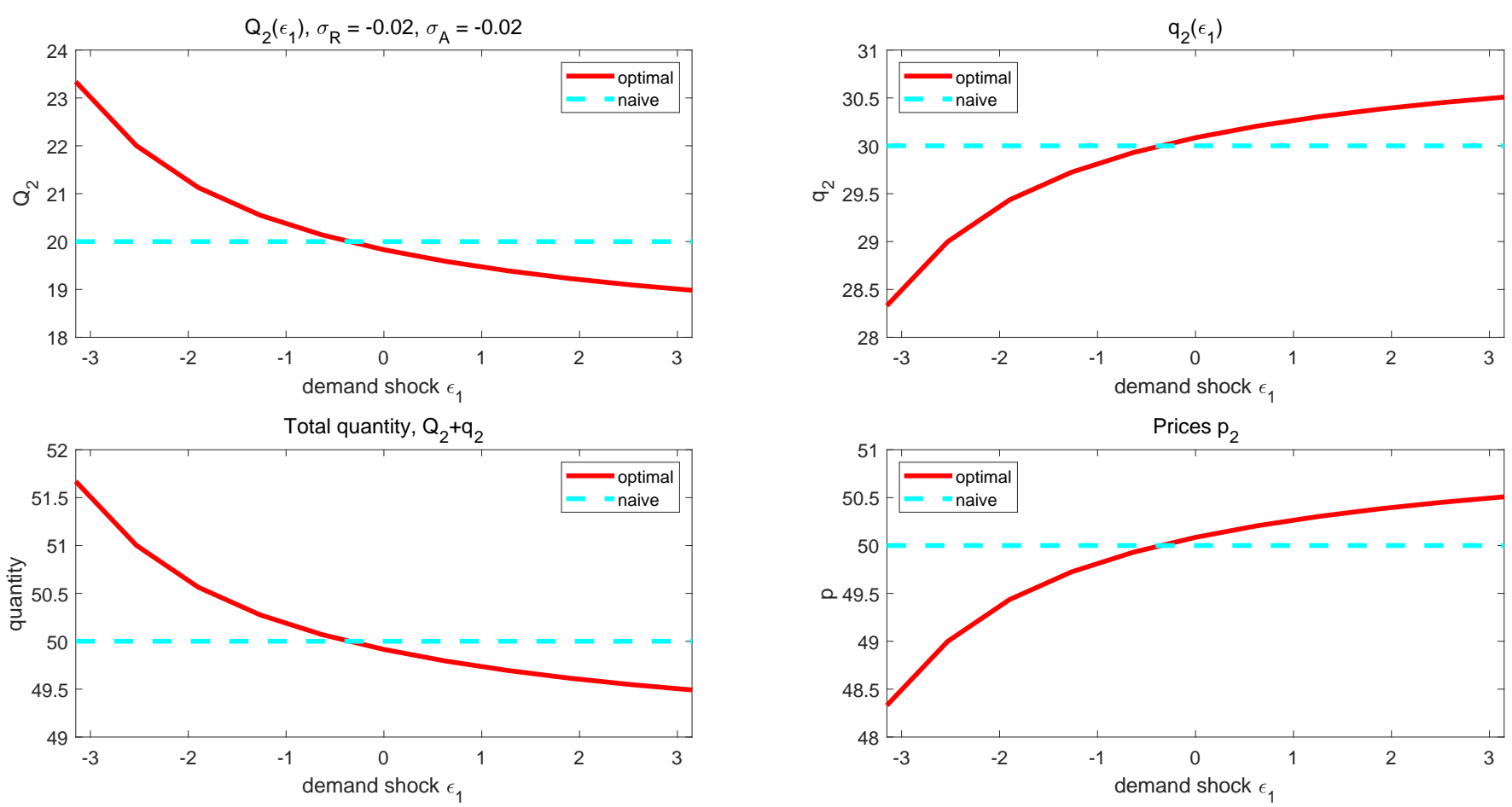

Figure 2: The top panels are displaying the quantities of the monopolist (left) and the fringe (right) at $t=2$. The bottom left panel depicts the total quantity in the market, and the bottom right panel the resulting price. The optimal quantities and prices are also contrasted to the quantities and induced prices that would prevail if we had a 'naive' monopolist. The 'naive' quantities and prices at $t=2$ are the same as the ones that would prevail if $\sigma_{A}=0$ and $\sigma_{R} \leq 0$.

parameter $\sigma(\equiv-1 / \theta)$. This indicates that the profits of the fringe fall relatively more than the profits of the monopolist in bad times.

Amplifying pessimism. We expect from Proposition 3 that the monopolist produces relatively more in bad times (reducing the fringe's profits), in order to make the fringe twist its beliefs towards bad profits states. This is clearly displayed in figure 2. The monopolist is increasing its quantity $Q_{2}\left(\epsilon_{1}\right)$ in bad times and decreases it in good times, leading to a relative reduction of prices in bad times and, consequently, to a decrease of competitive fringe quantities $\bar{q}_{2}\left(\epsilon_{1}\right)$ in bad times (with a respective increase in good times). Note that if there were no doubts about the model $\left(\sigma_{R}=\sigma_{A}=0\right)$, or if only the monopolist doubted the model and not the fringe $\left(\sigma_{R}<0, \sigma_{A}=0\right)$, the quantities at $t=2$ for both the monopolist and the fringe would be constant across shocks. ${ }^{10}$

A pessimistic fringe is cautious and assigns high probability mass on low demand shocks.

\footnotetext{
${ }^{10}$ This result is an outcome of the lack of persistence in the particular example and makes the contrast to the case of model uncertainty even sharper. See the Appendix for further details.
} 

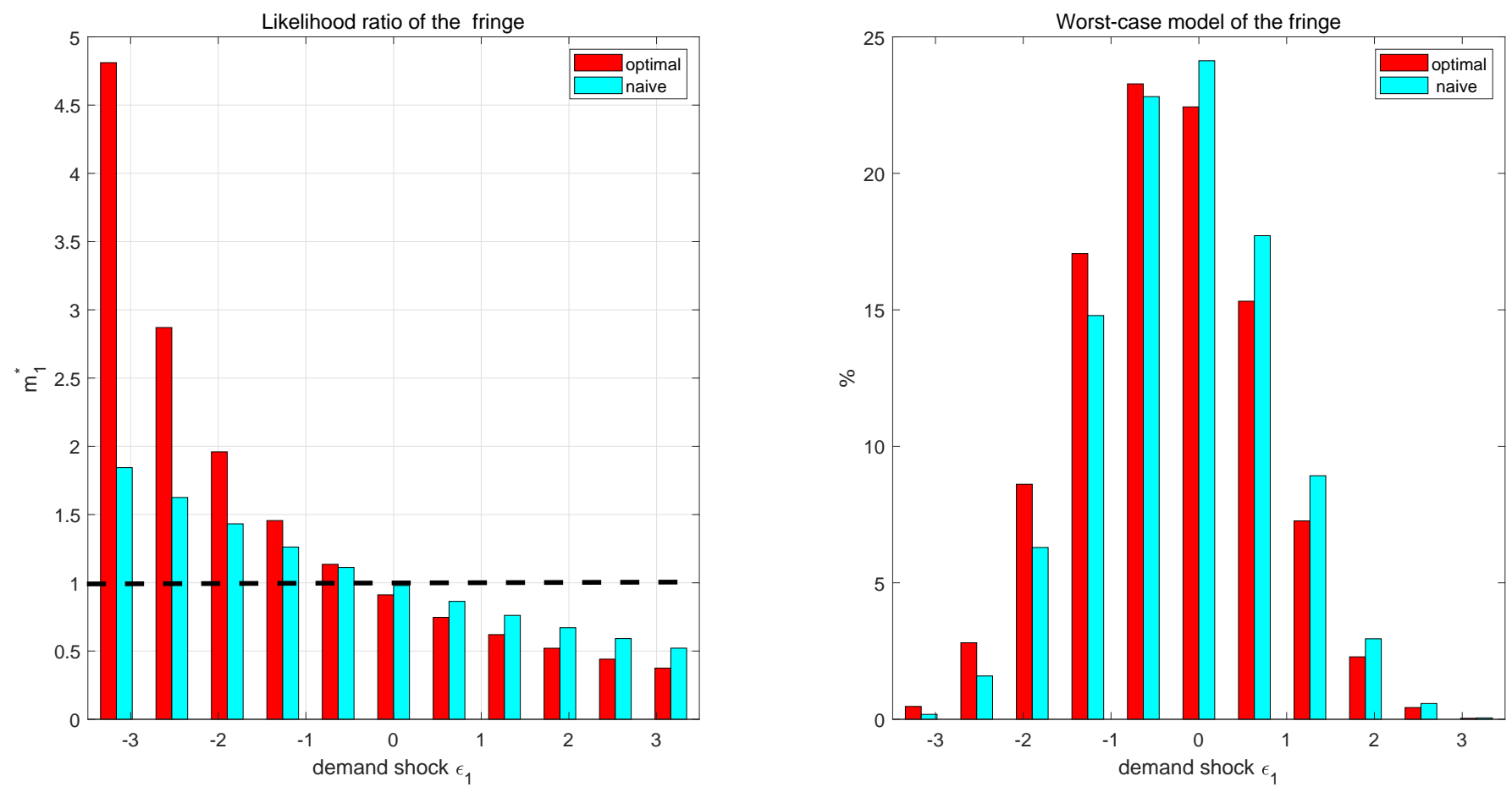

Figure 3: This graph contrasts the 'naive' worst-case beliefs of the fringe, that is, the beliefs that would emerge if the monopolist was not recognizing that the beliefs of the fringe were endogenous, to the optimal ones that entail expectation management (which were depicted in figure 1). The left panel depicts the likelihood ratios $m_{1}^{*}$ and the right panel the worst-case distributions.

What part of these endogenous pessimistic beliefs is an outcome of the actual expectation management from the side of the Stackelberg leader? To answer this question, consider the following thought experiment. Imagine that the monopolist did not recognize that the worst-case beliefs of the fringe were endogenous, and that he followed a 'naive' (or passive) policy, i.e. a policy that treated the fringe's beliefs as exogenously given. This thought experiment amounts to a 'belief-taking' assumption and mutes the expectation management through the multiplier $\tilde{\xi}_{t}^{V}$. As a result, the monopolist would choose constant quantities across shocks, as figure 2 demonstrates. ${ }^{11}$ Figure 3 contrasts the induced worst-case distribution of the fringe coming from the 'naive' policies to the optimal distribution that is stemming from the active expectation management, allowing us to see sharply the effects of pessimism per se versus the effects coming from the pessimistic expectation management. Relative to the 'naive' beliefs, the optimal beliefs assign a higher probability mass on low demand shocks. Consequently, the monopolist amplifies the worst-case beliefs of the fringe, above and beyond the fringe's inherent pessimism.

\footnotetext{
${ }^{11}$ The 'naive' quantities and prices at $t=2$ in figure 2 are exactly the same as the ones that would prevail if there was full confidence on the side of the fringe $\left(\sigma_{A}=0\right)$, and any confidence on the side of the monopolist, $\sigma_{R} \leq 0$. See the Appendix for details.
} 


\section{An optimal taxation problem}

In the second part of the paper we use as our laboratory the general equilibrium economy of Lucas and Stokey (1983). We attribute fears of model misspecification to both the policymaker (government) and the representative consumer (household). Drawing parallels with the first part of the paper, the policymaker corresponds to the monopolist and the representative household to the firm in the competitive fringe.

Time is discrete and the horizon is infinite. There is a single perishable good that can be allocated to private consumption $c_{t}$ or government consumption $g_{t}$. Government consumption is exogenous, stochastic, takes finite or countable values, and does not provide any utility. A linear production technology uses labor as input and converts one unit of labor to one unit of good.

Let $g^{t}=\left(g_{0}, \ldots, g_{t}\right)$ denote the partial history of government expenditures up to time $t$. There is a representative consumer that is endowed with one unit of time, works $h_{t}\left(g^{t}\right)$, enjoys leisure $l_{t}\left(g^{t}\right)=1-h_{t}\left(g^{t}\right)$ and consumes $c_{t}\left(g^{t}\right)$ at history $g^{t}$ for each $t \geq 0$. The notation indicates that the respective variables are measurable functions of $g^{t}$. The resource constraint of the economy reads

$$
c_{t}\left(g^{t}\right)+g_{t}=h_{t}\left(g^{t}\right) .
$$

Markets are complete and competitive. Competition makes the real wage $w_{t}\left(g^{t}\right)=1$ for all $t \geq 0$ and any history $g^{t}$. The government has no access to lump-sum taxes; instead it finances its time $t$ expenditures either by using a linear tax $\tau_{t}\left(g^{t}\right)$ on labor income or by issuing state-contingent debt $b_{t+1}\left(g_{t+1}, g^{t}\right)$ that is sold at price $p_{t}\left(g_{t+1}, g^{t}\right)$ at history $g^{t}$. This security pays one unit of the consumption good if government expenditures are $g_{t+1}$ next period and zero otherwise. The one-period government budget constraint at $t$ is

$$
b_{t}\left(g^{t}\right)+g_{t}=\tau_{t}\left(g^{t}\right) h_{t}\left(g^{t}\right)+\sum_{g_{t+1}} p_{t}\left(g_{t+1} \mid g^{t}\right) b_{t+1}\left(g_{t+1}, g^{t}\right)
$$

Equivalently, using the proper no-Ponzi game condition, we get the single intertemporal budget constraint

$$
b_{0}+\sum_{t=0}^{\infty} \sum_{g^{t}} q_{t}\left(g^{t}\right) g_{t} \leq \sum_{t=0}^{\infty} \sum_{g^{t}} q_{t}\left(g^{t}\right) \tau_{t}\left(g^{t}\right) h_{t}\left(g^{t}\right),
$$

where $q_{t}\left(g^{t}\right)$ the history-contingent prices of Arrow-Debreu contracts trade at $t=0 .^{12}$

\footnotetext{
${ }^{12}$ Note also that in order to avoid introducing new notation, we use $p_{t}$ and $q_{t}$ in a different way that in the first part of the paper.
} 


\subsection{Model misspecification}

The representative consumer and the government share a reference probability model in terms of a sequence of joint densities $\pi_{t}\left(g^{t}\right)$ over histories $g^{t}$. The expectation operator with respect to the reference model is denoted again by $E$. Uncertainty at $t=0$ has been realized, so $\pi_{0}\left(g_{0}\right)=1$. Both the consumer and the government fear that the reference model is misspecified and consider alternative probability models that are absolutely continuous with respect to the reference model over finite time intervals. As in the first part of the paper, we use positive martingales in order to express these alternative models. Moreover, we follow the same notation for the likelihood ratios of the household-follower and the policymaker-leader as we did for the competitive fringe and the monopolist respectively. So we have the following:

Representative consumer. The alternative models of the household can be expressed as a non-negative random variable $M_{t}$, or else, likelihood ratio, with $E M_{t}=1$ and $E_{t} M_{t+1}=M_{t}$. The initial value is set to unity, $M_{0} \equiv 1$. The respective conditional likelihood ratio is $m_{t+1}=$ $M_{t+1} / M_{t}$ with $E_{t} m_{t+1}=1$.

Government. The alternative models of the government are expressed as a non-negative random variable $N_{t}$ with $E N_{t}=1$ and $N_{0} \equiv 1$. The likelihood ratio $N_{t}$ is a martingale with respect to the reference model, so $E_{t} N_{t+1}=N_{t}$. The respective conditional likelihood ratio is $n_{t+1}=N_{t+1} / N_{t}$, with $E_{t} n_{t+1}=1$.

\subsection{Ambiguity aversion}

Both the consumer and the government are averse to model ambiguity. We represent this aversion by using the multiplier preferences of Hansen and Sargent (2001) and Hansen et al. (2006).

Representative consumer. The consumer ranks consumption and leisure plans using the following criterion:

$\min _{m_{t+1} \geq 0, M_{t} \geq 0} \sum_{t=0}^{\infty} \beta^{t} \sum_{g^{t}} \pi_{t}\left(g^{t}\right) M_{t}\left(g^{t}\right) U\left(c_{t}\left(g^{t}\right), 1-h_{t}\left(g^{t}\right)\right)+\beta \theta_{A} \sum_{t=0}^{\infty} \beta^{t} \sum_{g^{t}} \pi_{t}\left(g^{t}\right) M_{t}\left(g^{t}\right) \varepsilon_{t}\left(m_{t+1}\left(g^{t+1}\right)\right)$,

subject to $E_{t} m_{t+1}=1$ and $M_{t+1}=m_{t+1} M_{t}$, with $M_{0}=1$ and $0<\theta_{A} \leq \infty$. The period utility $U\left(c_{t}, 1-h_{t}\right)$ satisfies the typical monotonicity and concavity assumptions. The positive parameter $\theta_{A}$ is a penalty parameter that measures fear of model misspecification. 
According to (52), the representative household evaluates expected utility under the alternative probability models, as captured by $M_{t}$, and shows its aversion to model ambiguity by considering the model that furnishes the worst utility. Deviations from the reference model are penalized in terms of a measure of discounted relative entropy. Higher values of the parameter $\theta_{A}$ represent more confidence in the reference model $\pi_{t}$. Full confidence is captured by $\theta_{A}=\infty$, which reduces the above preferences to the expected utility preferences of the Lucas and Stokey household.

Government. The government's preferences are described by

$\min _{n_{t+1} \geq 0, N_{t} \geq 0} \sum_{t=0}^{\infty} \beta^{t} \sum_{g^{t}} \pi_{t}\left(g^{t}\right) N_{t}\left(g^{t}\right) U\left(c_{t}\left(g^{t}\right), 1-h_{t}\left(g^{t}\right)\right)+\beta \theta_{R} \sum_{t=0}^{\infty} \beta^{t} \sum_{g^{t}} \pi_{t}\left(g^{t}\right) N_{t}\left(g^{t}\right) \varepsilon_{t}\left(n_{t+1}\left(g^{t+1}\right)\right)$

subject to $E_{t} n_{t+1}=1$ and $N_{t+1}=n_{t+1} N_{t}$, with $N_{0}=1$ and $0<\theta_{R} \leq \infty$. Similarly, $\theta_{R}$ captures the policymaker's confidence in the reference probability model.

If $\theta_{R}=\theta_{A}$, then the policymaker becomes a "benevolent" planner that adopts the preferences of the household. This is in contrast to the first part of the paper, where equality of the penalty parameters $\theta_{i}, i=R, A$ was not sufficient to equalize the objectives of the monopolist and the competitive fringe, since the return functions were different. In the case of $\theta_{R} \neq \theta_{A}$, the policymaker exhibits paternalism, that is, he imposes his own evaluation of the utility that the household is deriving from a stochastic stream of consumption and leisure. We don't take a stance on the criterion of the government and we allow the policymaker to doubt the model less $\left(\theta_{R}>\theta_{A}\right)$, the same $\left(\theta_{R}=\theta_{A}\right)$, or more than the household $\left(\theta_{R}<\theta_{A}\right)$.

\subsection{The representative household's problem}

The problem of the consumer is

$$
\begin{aligned}
\max _{c_{t}, h_{t}} \min _{M_{t} \geq 0, m_{t+1} \geq 0} & \sum_{t=0}^{\infty} \beta^{t} \sum_{g^{t}} \pi_{t}\left(g^{t}\right) M_{t}\left(g^{t}\right)\left[U\left(c_{t}\left(g^{t}\right), 1-h_{t}\left(g^{t}\right)\right)\right. \\
& \left.+\theta_{A} \beta \sum_{g_{t+1}} \pi_{t+1}\left(g_{t+1} \mid g^{t}\right) m_{t+1}\left(g^{t+1}\right) \ln m_{t+1}\left(g^{t+1}\right)\right]
\end{aligned}
$$


subject to

$$
\begin{aligned}
\sum_{t=0}^{\infty} \sum_{g^{t}} q_{t}\left(g^{t}\right) c_{t}\left(g^{t}\right) & \leq \sum_{t=0}^{\infty} \sum_{g^{t}} q_{t}\left(g^{t}\right)\left(1-\tau_{t}\left(g^{t}\right)\right) h_{t}\left(g^{t}\right)+b_{0} \\
c_{t}\left(g^{t}\right) & \geq 0, h_{t}\left(g^{t}\right) \in[0,1] \forall t, g^{t} \\
M_{t+1}\left(g^{t+1}\right) & =m_{t+1}\left(g^{t+1}\right) M_{t}\left(g^{t}\right), M_{0}=1 \forall t, g^{t} \\
\sum_{g_{t+1}} \pi_{t+1}\left(g_{t+1} \mid g^{t}\right) m_{t+1}\left(g^{t+1}\right) & =1, \forall t, g^{t}
\end{aligned}
$$

Inequality (54) is the intertemporal budget constraint of the household. The right side is the discounted present value of after-tax labor income plus an initial asset position $b_{0}$ that can assume positive (denoting government debt) or negative (denoting government assets) values.

\subsection{Worst-case beliefs}

The optimal conditional likelihood ratio takes the familiar exponentially twisting form:

$$
m_{t+1}^{*}\left(g^{t+1}\right)=\frac{\exp \left(-\frac{V_{t+1}\left(g^{t+1}\right)}{\theta_{A}}\right)}{\sum_{g_{t+1}} \pi_{t+1}\left(g_{t+1} \mid g^{t}\right) \exp \left(-\frac{V_{t+1}\left(g^{t+1}\right)}{\theta_{A}}\right)}, \text { all } t \geq 0, g^{t}
$$

where the asterisks denote optimal values from the minimization problem and $V_{t}$ is the household's utility under the worst-case measure, which, as in the first part of the paper, follows the risk-sensitive recursion

$$
V_{t}=U\left(c_{t}, 1-h_{t}\right)+\frac{\beta}{\sigma_{A}} \ln E_{t}\left(\exp \left(\sigma_{A} V_{t+1}\right)\right)
$$

where $\sigma_{A} \equiv-1 / \theta_{A} \leq 0$. Equation (58) summarizes how a cautious household forms pessimistic beliefs. The household assigns high probability (relative to the reference model) to events with low continuation utilities $V_{t+1}$, and low probability to events with high utility $V_{t+1}$.

Lastly, the law of motion in (56) becomes

$$
M_{t+1}^{*}=\frac{\exp \left(\sigma_{A} V_{t+1}\left(g^{t+1}\right)\right)}{\sum_{g_{t+1}} \pi_{t+1}\left(g_{t+1} \mid g^{t}\right) \exp \left(\sigma_{A} V_{t+1}\left(g^{t+1}\right)\right)} M_{t}^{*}, \quad M_{0} \equiv 1 .
$$

\subsection{Labor supply, asset choice and equilibrium}

The labor supply of the household is determined by equalizing the marginal rate of substitution between consumption and leisure to the after-tax wage, 


$$
\frac{U_{l}\left(g^{t}\right)}{U_{c}\left(g^{t}\right)}=1-\tau_{t}\left(g^{t}\right) .
$$

The asset choice of the household is determined by condition

$$
q_{t}\left(g^{t}\right)=\beta^{t} \pi_{t}\left(g^{t}\right) M_{t}^{*}\left(g^{t}\right) \frac{U_{c}\left(g^{t}\right)}{U_{c}\left(g_{0}\right)},
$$

which equalizes the intertemporal rate of substitution between consumption at time $t$ and consumption at the initial period to the price of an Arrow-Debreu contract. The price at $t=0$ is normalized to unity, $q_{0}=1$. Similarly, the optimality condition when there is trade in statecontingent Arrow securities is

$$
p_{t}\left(g_{t+1}, g^{t}\right)=\beta \pi_{t+1}\left(g_{t+1} \mid g^{t}\right) \underbrace{\frac{\exp \left(\sigma_{A} V_{t+1}\left(g^{t+1}\right)\right)}{\sum_{g_{t+1}} \pi_{t+1}\left(g_{t+1} \mid g^{t}\right) \exp \left(\sigma_{A} V_{t+1}\left(g^{t+1}\right)\right)}}_{m_{t+1}^{*}} \frac{U_{c}\left(g^{t+1}\right)}{U_{c}\left(g^{t}\right)} .
$$

The expression for the equilibrium price of a state-contingent claim (63) involves the ratio of marginal utilities, and, most importantly, the pessimistic evaluation of the likelihood of the particular contingency, $m_{t+1}^{*}$, which depends on continuation utilities. By affecting utility, the policymaker's choices influence the household's worst-case beliefs and ultimately the equilibrium prices of government debt. This is the core of pessimistic expectation management in the second part of the paper and a channel that was first analyzed in Karantounias (2013).

Definition 1. A competitive equilibrium is a consumption-labor allocation $(c, h)$, distortions to beliefs $(m, M)$, a price system $q$, and a government policy $(g, \tau)$ such that $(a)$ given $(q, \tau),(c, h)$ and $(m, M)$ solve the household's problem, and (b) markets clear, so that $c_{t}\left(g^{t}\right)+g_{t}=h_{t}\left(g^{t}\right) \forall t, g^{t}$.

\section{Disagreement and pessimistic expectation management}

We proceed now to the analysis of optimal fiscal policy. There are two forces that are prevalent in the analysis: the discrepancy (if any) in the pessimistic beliefs of the leader-policymaker and the follower-household, and the management of the endogenous pessimistic expectations of the household. For the particular optimal taxation application, the discrepancy in the pessimistic evaluations is related to the paternalism of the policymaker, whereas the pessimistic expectation management is related, as in Karantounias (2013), to the manipulation of equilibrium prices of state-contingent debt with the ultimate target to make debt issuance cheaper. 


\section{$5.1 \quad$ Fiscal policy problem}

The government chooses taxes and state-contingent debt at $t=0$ in order to maximize the government's welfare criterion (53). We use the primal approach of Lucas and Stokey (1983) and posit a policymaker who chooses under commitment allocations subject to the resource constraint (49) and implementability constraints imposed by the competitive equilibrium.

Problem 3. The government's problem is

$$
\max _{\left(c, h, M^{*}, V\right)} \min _{n, N \geq 0} \sum_{t=0}^{\infty} \beta^{t} \sum_{g^{t}} \pi_{t}\left(g^{t}\right) N_{t}\left(g^{t}\right)\left[U\left(c_{t}\left(g^{t}\right), 1-h_{t}\left(g^{t}\right)\right)+\beta \theta_{R} \varepsilon_{t}\left(n_{t+1}\right)\right]
$$

subject to

$$
\begin{aligned}
& \sum_{t=0}^{\infty} \beta^{t} \sum_{g^{t}} \pi_{t}\left(g^{t}\right) M_{t}^{*}\left(g^{t}\right)\left[U_{c}\left(g^{t}\right) c_{t}\left(g^{t}\right)-U_{l}\left(g^{t}\right) h_{t}\left(g^{t}\right)\right]=U_{c}\left(g_{0}\right) b_{0} \\
& c_{t}\left(g^{t}\right)+g_{t}=h_{t}\left(g^{t}\right), \forall t, g^{t} \\
& M_{t+1}^{*}\left(g^{t+1}\right)=\frac{\exp \left(\sigma_{A} V_{t+1}\left(g^{t+1}\right)\right)}{\sum_{g_{t+1}} \pi_{t+1}\left(g_{t+1} \mid g^{t}\right) \exp \left(\sigma_{A} V_{t+1}\left(g^{t+1}\right)\right)} M_{t}^{*}\left(g^{t}\right), \forall t, g^{t}, M_{0}\left(g_{0}\right) \equiv 1, \\
& V_{t}\left(g^{t}\right)=U\left(c_{t}\left(g^{t}\right), 1-h_{t}\left(g^{t}\right)\right)+\frac{\beta}{\sigma_{A}} \ln \sum_{g_{t+1}} \pi_{t+1}\left(g_{t+1} \mid g^{t}\right) \exp \left(\sigma_{A} V_{t+1}\left(g^{t+1}\right)\right), \\
& \forall t, g^{t}, t \geq 1 \\
& N_{t+1}\left(g^{t+1}\right)=n_{t+1}\left(g^{t+1}\right) N_{t}\left(g^{t}\right), \forall t, g^{t}, N_{0}\left(g_{0}\right) \equiv 1 \\
& \sum_{g_{t+1}} \pi_{t+1}\left(g_{t+1} \mid g^{t}\right) n_{t+1}\left(g^{t+1}\right)=1
\end{aligned}
$$

Proof. The competitive equilibrium is characterized fully by the resource constraint, the household's two Euler equations, the intertemporal budget constraint (54) (which holds with equality), the law of motion of the household's worst-case belief distortions (60), and the recursion for $V_{t}$ in (59), which helps determine the endogenous pessimistic beliefs. Use (61) and (62) to substitute for prices and after-tax wages in the intertemporal budget constraint to obtain (64).

The presence of the household's pessimistic beliefs in the implementability constraint (64) contributes two additional implementability constraints to those already in Lucas and Stokey (1983): the law of motion of the endogenous likelihood ratio $M_{t}^{*}(66)$, and the household's utility recursion (67).

\subsection{Disagreement}

Government's worst-case beliefs. The worst-case beliefs of the policymaker are given by the optimality conditions of the minimization problem in problem 3. The optimal conditional 
likelihood ratio of the government takes the form

$$
n_{t+1}^{*}\left(g^{t+1}\right)=\frac{\exp \left(-\frac{W_{t+1}\left(g^{t+1}\right)}{\theta_{R}}\right)}{\sum_{g_{t+1}} \pi_{t+1}\left(g_{t+1} \mid g^{t}\right) \exp \left(-\frac{W_{t+1}\left(g^{t+1}\right)}{\theta_{R}}\right)}
$$

with $W_{t}$ following the recursion

$$
W_{t}=U\left(c_{t}, 1-h_{t}\right)+\frac{\beta}{\sigma_{R}} \ln E_{t} \exp \left(\sigma_{R} W_{t+1}\right)
$$

where $\sigma_{R} \equiv-1 / \theta_{R}$. $W_{t+1}$ represents the conservative evaluation of the utility of the policymaker. As expected, the policymaker assigns higher probability to events that provide low continuation utility $W_{t+1}$.

Belief ratio. The worst-case unconditional likelihood ratio of the policymaker has a law of motion $N_{t+1}^{*}=n_{t+1}^{*} N_{t}^{*}, N_{0}=1$. We can define the belief ratio $\Lambda_{t} \equiv M_{t}^{*} / N_{t}^{*}$ as the ratio of the martingales $M_{t}^{*}, N_{t}^{*}$. The belief ratio follows law of motion

$$
\Lambda_{t}=\frac{m_{t}^{*}}{n_{t}^{*}} \cdot \Lambda_{t-1}=\frac{\exp \left(\sigma_{A} V_{t+1}\right) / E_{t} \exp \left(\sigma_{A} V_{t+1}\right)}{\exp \left(\sigma_{R} W_{t+1}\right) / E_{t} \exp \left(\sigma_{R} W_{t+1}\right)} \cdot \Lambda_{t-1}, t \geq 1
$$

with $\Lambda_{0} \equiv 1$, and $V_{t}$ and $W_{t}$ following recursions (59) and (71) respectively. The ratio $\Lambda_{t}$ captures the disagreement that the household and the policymaker express about the likelihood of a particular contingency. A high $\Lambda_{t}$ at a history $g^{t}$ denotes a contingency on which the pessimistic household assigns a higher probability than the policymaker. Similarly, if $\Lambda_{t}$ is low, then the policymaker assigns a higher probability than the household. The disagreement in beliefs matters because it implies a different welfare ranking of competitive equilibrium allocations. Note that if $\theta_{R}=\theta_{A}$, recursion (71) becomes the same as the utility recursion (59) for the representative household and therefore, we have $W_{t}=V_{t}$ and $N_{t}^{*}=M_{t}^{*}$. The belief ratio becomes then identically unity, $\Lambda_{t}\left(g^{t}\right)=1, \forall t, g^{t}$.

\subsection{Optimal tax rate}

To see the forces of paternalism and pessimistic expectation management, we have to consider the optimal tax rate.

Proposition 4. ('Optimal tax rate')

Assume that initial assets are not large enough to finance spending without resorting to distortionary taxation and let $\Phi>0$ denote the multiplier on the implementability constraint 
(64). Let $\tilde{\xi}_{t}$ denote the (normalized with the policymaker's likelihood ratio $N_{t}^{*}$ ) multiplier on the household's utility recursion (67) in problem 3.

- The optimal tax rate for $t \geq 1$ is

$$
\tau_{t}=\frac{\Phi \Lambda_{t}\left(\epsilon_{c c, t}+\epsilon_{c h, t}+\epsilon_{h h, t}+\epsilon_{h c, t}\right)}{1+\tilde{\xi}_{t}+\Phi \Lambda_{t}\left(1+\epsilon_{h h, t}+\epsilon_{h c, t}\right)}
$$

where $\epsilon_{c c, t} \equiv-U_{c c} c_{t} / U_{c}, \epsilon_{c h, t} \equiv U_{c l} h_{t} / U_{c}, \epsilon_{h h, t} \equiv-U_{l l} h_{t} / U_{l}$ and $\epsilon_{h c, t} \equiv U_{c l} c_{t} / U_{l}$, the own and cross elasticities of the marginal utility of consumption and the marginal disutility of labor respectively. If $U_{c l} \geq 0$, then $\tau_{t} \geq 0$.

- Consider the following two examples:

1. (Power function in $h)$ Let $U(c, 1-h)=\frac{c^{1-\gamma}-1}{1-\gamma}-a_{h} \frac{h^{1+\phi_{h}}}{1+\phi_{h}}$. Then, (173) implies

$$
\tau_{t}=\frac{\Phi \Lambda_{t}\left(\gamma+\phi_{h}\right)}{1+\tilde{\xi}_{t}+\Phi \Lambda_{t}\left(1+\phi_{h}\right)}
$$

2. (Power function in l) Let $U(c, l)=\frac{c^{1-\gamma}-1}{1-\gamma}+a_{l} \frac{l^{1-\psi}-1}{1-\psi}$. Then, (73) implies

$$
\tau_{t}=\frac{\Phi \Lambda_{t}\left(\gamma+\psi \frac{h_{t}}{1-h_{t}}\right)}{1+\tilde{\xi}_{t}+\Phi \Lambda_{t}\left(1+\psi \frac{h_{t}}{1-h_{t}}\right)} .
$$

Proof. See the Appendix.

Shutting down doubts about the model $\left(\sigma_{R}=\sigma_{A}=0\right)$ delivers the classic Lucas and Stokey (1983) environment. There is no room for the policymaker to manipulate the worst-case beliefs of the household through continuation utilities, so $\tilde{\xi}_{t}=0$. Furthermore, with full confidence in the model, we have $N_{t}^{*}=M_{t}^{*}=1$ so the belief ratio becomes identically unity, $\Lambda_{t}=1, \forall t$. Any variation in the tax rate (73) is coming from variation in the curvature of the period utility function, as captured by the elasticities $\epsilon_{i j}, i, j=c, h$. If these elasticities were constant, like in example (74), we would have perfect tax-smoothing.

With ambiguity though $\left(\sigma_{R}<0, \sigma_{A}<0\right)$, in addition to the elasticity channel, there is variation in the tax rate coming from the management of the pessimistic expectations of the household as captured by $\tilde{\xi}_{t}$. Additionally, in the case of paternalism $\left(\sigma_{R} \neq \sigma_{A}\right)$, there is further action in the tax rate coming from the belief ratio $\Lambda_{t}$. We turn now to the analysis of these mechanisms. 


\subsection{Pessimistic expectation management}

Let $\mu_{t}$ denote the multiplier of the law of motion of the household's worst-case likelihood ratio (66). The first-order condition with respect to $V_{t}$ delivers the following law of motion for $\tilde{\xi}_{t}$,

$$
\tilde{\xi}_{t}=\underbrace{\sigma_{A} \frac{m_{t}^{*}}{n_{t}^{*}}\left[\mu_{t}-E_{t-1} m_{t}^{*} \mu_{t}\right] \Lambda_{t-1}}_{\text {value of reducing } m_{t}^{*}}+\frac{m_{t}^{*}}{n_{t}^{*}} \tilde{\xi}_{t-1}, t \geq 1, \tilde{\xi}_{0} \equiv 0 .
$$

Recall that the policymaker has no need to keep track of any utility promises at $t=0$ (since uncertainty is realized at $t=0$ ), which explains why $\tilde{\xi}_{0}=0$. It is easy to see that $\tilde{\xi}_{t}$ is a martingale with respect to the worst-case beliefs of the policymaker $\pi_{t} \cdot N_{t}^{*}, E_{t} n_{t+1}^{*} \tilde{\xi}_{t+1}=\tilde{\xi}_{t}$, a fact that induces persistence. So the mean value of the multiplier is zero, $E N_{t}^{*} \tilde{\xi}_{t}=0$, and it can take positive and negative values.

Notice the similarity of (76) with the respective law of motion of $\tilde{\xi}_{t}^{V}$ in the competitive fringe application (32). As in the first part of the paper, the multiplier $\tilde{\xi}_{t}$ captures the management of the pessimistic expectations of the follower; the representative household in the particular economy. An increase in the household utility $V_{t}$ makes the household tilt less its beliefs, reducing $m_{t}^{*}$, with a net effect of $\mu_{t}-E_{t-1} m_{t}^{*} \mu_{t}$. What is the benefit to the policymaker of an increase or decrease of the likelihood ratio $m_{t}^{*}$ ? In the Appendix, we show that $\mu_{t}$ is proportional to the debt position of the government, properly scaled with marginal utility,

$$
\mu_{t}\left(g^{t}\right)=\Phi U_{c}\left(g^{t}\right) b_{t}\left(g^{t}\right)
$$

Using (77), we can rewrite (76) as

$$
\tilde{\xi}_{t}=\sigma_{A} \frac{m_{t}^{*}}{n_{t}^{*}} \Phi\left[U_{c t} b_{t}-E_{t-1} m_{t}^{*} U_{c t} b_{t}\right] \Lambda_{t-1}+\frac{m_{t}^{*}}{n_{t}^{*}} \tilde{\xi}_{t-1}
$$

Moreover, if we shut down the paternalism of the government by setting $\sigma_{R}=\sigma_{A}=\bar{\sigma}$, (78) simplifies to

$$
\tilde{\xi}_{t}=\bar{\sigma} \Phi\left[U_{c t} b_{t}-E_{t-1} m_{t}^{*} U_{c t} b_{t}\right]+\tilde{\xi}_{t-1},
$$

since in that case the ratio of conditional and unconditional beliefs becomes unity, $\frac{m_{t}^{*}}{n_{t}^{*}}=\Lambda_{t}=$ $1, \forall t$. 
Making debt cheaper. Equations (77) and (78) indicate that the policymaker wants to increase the pessimistic likelihood ratio of the household (by decreasing $V_{t}$ ) at states of the world against which he issues relatively high debt in marginal utility units, $U_{c t} b_{t}>E_{t-1} m_{t}^{*} U_{c t} b_{t}$. Similarly, the policymaker wants to decrease the likelihood ratio (by increasing $V_{t}$ ) at states of the world against which he issues relatively low debt in marginal utility units (or buys assets), $U_{c t} b_{t}<E_{t-1} m_{t}^{*} b_{t}$. What is the mechanism here? By increasing $m_{t}^{*}$, the policymaker makes the equilibrium price of government debt higher, as seen in (63). This is beneficial when the policymaker is selling debt, as seen in (77). In other words, the policymaker reduces effectively the interest rate on the debt that he is selling, making debt cheaper. This relaxes the government budget by increasing revenue from debt issuance and increases welfare. Recall though that likelihood ratios are interconnected, since they have to integrate to unity, so the net effect of decreasing $V_{t}$ is captured by the relative debt position $U_{c t} b_{t}-E_{t-1} m_{t}^{*} U_{c t} b_{t}$. In a similar manner, when debt in marginal utility units is relatively low, the policymaker has an incentive to decrease the likelihood ratio and decrease the price of the state-contingent claim that is bought. Thus, the rate on the assets that the government acquires increases, relaxing the pressure on the government budget. ${ }^{13}$

Tax rate and price manipulation. To see effectively the same mechanism in terms of the tax rate, shut off paternalism $\left(\sigma_{R}=\sigma_{A}=\bar{\sigma}<0\right)$ and consider the evolution of the tax rate in (79). For states of the world for which debt is relatively high $\left(U_{c t} b_{t}>E_{t-1} m_{t}^{*} U_{c t} b_{t}\right), \tilde{\xi}_{t}$ falls, $\tilde{\xi}_{t}<\tilde{\xi}_{t-1}$. A decrease in $\tilde{\xi}_{t}$ increases the tax rate, all else equal, as seen in (73). This is just another manifestation of the price manipulation mechanism. The policymaker increases the tax rate exactly at these states, against which it becomes cheaper to issue debt. Intuitively, by increasing the tax rate for high-debt states, the policymaker reduces the utility of the household, making it assign higher probability against these states, increasing therefore the price of debt sold. Instead, for low-debt (or asset) states $\left(U_{c t} b_{t}<E_{t-1} m_{t}^{*} U_{c t} b_{t}\right)$, $\tilde{\xi}_{t}$ increases, $\tilde{\xi}_{t}>\tilde{\xi}_{t-1}$, decreasing therefore the tax rate. So for low-debt states, the policymaker reduces the tax rate and increases utility in order to reduce the current price of claims that he acquires.

Mitigating pessimism. The previous analysis did not associate the high or low debt states of the world to the type of shocks they are associated with. Due to complete markets, we expect that the government hedges fiscal shocks by issuing more debt against low spending shocks ('good' times), which is to be paid back by running surpluses, and by buying assets against 'bad' times in order to insure against high government spending. So, following the price manipulation logic, we expect that the government is making debt cheaper by increasing taxes

\footnotetext{
${ }^{13}$ With the exception of the government's worst-case beliefs, the mechanism in this section is similar to Karantounias (2013).
} 
Table 1: Summary of expectation management of the follower.

\begin{tabular}{c|c|c|c}
\hline \hline Environment & $\begin{array}{c}\text { Object determining } \\
\text { beliefs }\end{array}$ & $\begin{array}{c}\text { Shadow value of } \\
\text { increasing } m_{t}^{*}\end{array}$ & $\begin{array}{c}\text { Increase } m_{t}^{*} \\
\text { in }\end{array}$ \\
\hline Large firm facing & Present value of & $\mu_{t}=C_{\epsilon} \epsilon_{t} \tilde{\xi}_{t-1}^{i} N_{t-1}^{*}$ & 'bad' times \\
a competitive fringe & profits & $\tilde{\xi}_{t}^{i}<0$ & $\left(\right.$ low $\left.\epsilon_{t}\right)$ \\
Economy with & Utility & $\mu_{t}=\Phi U_{c t} b_{t}$ & 'good' times \\
linear labor taxes & & $\Phi>0$ & $\left(\right.$ low $\left.g_{t}\right)$ \\
\hline \hline
\end{tabular}

This table contrasts the pessimistic expectation management in the two environments of the paper (through $\tilde{\xi}_{t}^{V}$ and $\tilde{\xi}_{t}$ respectively). For the industry problem, we are reporting here for simplicity only the case with zero adjustment costs, which allowed the sharp illustration of belief amplification.

against good times of low spending shocks and by decreasing tax rates against bad times of high spending shocks. But the cautious consumer is assigning lower probability than the reference model in good times and higher probability in bad times. Thus, by taxing more and reducing utility in good times, the policymaker is mitigating the pessimism of the household, in contrast to the belief amplification we highlighted in the first part of the paper. Table 1 provides a useful summary of pessimistic expectation management in the two economies.

\subsection{Putting the two forces together}

The analysis in the previous section would be sufficient if we constrained ourselves to a benevolent planner $\left(\sigma_{R}=\sigma_{A}\right)$. Turning to the analysis of paternalism $\left(\sigma_{R} \neq \sigma_{A}\right)$, we can see sharply the effects of the disagreement in beliefs on the optimal plan, in contrast to the first part of the paper.

Disagreement and the tax rate. Consider the optimal tax rate (73) in proposition 4. Keeping everything else equal, we see that an increase in the belief ratio $\Lambda_{t}$ increases the tax rate $\tau_{t}{ }^{14}$ The reason behind this outcome is intuitive. An increase in $\Lambda_{t}\left(g^{t}\right)$ means that the household considers history $g^{t}$ more probable than the government. Therefore, the welfare loss associated with a distortionary tax is small in the eyes of the paternalistic government, since these histories are not considered probable. Consequently, the paternalistic government has an incentive to tax

\footnotetext{
${ }^{14}$ The belief ratio $\Lambda_{t}$ affects also the evolution of the multiplier $\tilde{\xi}_{t}$, as seen by (76). Solve backwards $\tilde{\xi}_{t}$ to get $\tilde{\xi}_{t}=\sigma_{A} \Lambda_{t} \sum_{i=1}^{t} \eta_{i}$, where $\eta_{i} \equiv \mu_{i}-E_{i-1} m_{i}^{*} \mu_{i}, i \geq 1$. Thus, the optimal tax rate in (73) can be written as $\tau_{t}=\frac{\Phi \Lambda_{t}\left(\epsilon_{c c, t}+\epsilon_{c h, t}+\epsilon_{h h, t}+\epsilon_{h c, t}\right)}{1+\Lambda_{t}\left[\sigma_{A} \sum_{i} \eta_{i}+\Phi\left(1+\epsilon_{h h, t}+\epsilon_{h c, t}\right)\right]}$. Differentiate now $\tau_{t}$ with respect to $\Lambda_{t}$, keeping elasticities constant (or just consider example (74)), to get $\frac{\partial \tau_{t}}{\partial \Lambda_{t}}=\frac{\Phi\left(\epsilon_{c c, t}+\epsilon_{c h, t}+\epsilon_{h h, t}+\epsilon_{h c, t}\right)}{\left(1+\tilde{\xi}_{t}+\Phi \Lambda_{t}\left(1+\epsilon_{h h, t}+\epsilon_{h c, t}\right)\right)^{2}}>0$, for $U_{c l} \geq 0$.
} 
more states of the world that is does not consider probable (relative to the household) and less states of the world that it considers more probable than the household.

Paternalism in 'good' and 'bad' times. To elaborate on the previous paragraph, the effects of paternalism depend on the relative pessimism of the government versus the household's. Consider for example the case where the government doubts the model less than the household, so that $\sigma_{A}<\sigma_{R}$ and fix the history of shocks up to $t-1$. Then, we expect that the government is twisting less its beliefs (relative to the household) towards bad times of high government spending at $t$, so we expect $\Lambda_{t}$ to be high when $g_{t}$ is high. Similarly, we expect the belief ratio to be low in good times, i.e. when $g_{t}$ is low. Thus, a paternalistic government that is less pessimistic than the household would try to tax more in bad times and less in good times. Following exactly the same logic, we expect the opposite result when the government is more pessimistic than the household, $\sigma_{R}<\sigma_{A}$, i.e. we expect the government to impose smaller taxes in bad times (which are considered to be very probable, so the welfare loss of a given tax is high) and higher taxes in good times.

Opposite or same direction? Putting now the two forces of paternalism and expectation management together, we see that the direction of tax rate depends on the strength of the government's versus the households doubts. If the government doubts the model less than the household $\left(\sigma_{A}<\sigma_{R}\right)$, then we expect that it will tax more in bad times and less in good times. But the price manipulation through the expectation management commands to tax less in bad times and more in good times. Consequently, the two effects operate in the opposite direction and the final response of the tax rate depends on the size of the two effects. This case nests effectively the case that was analyzed in Karantounias (2013), who considered a government with full confidence in the model, $\sigma_{A}<\sigma_{R}=0 .{ }^{15}$ However, the fact that paternalism acts in the opposite direction than price manipulation is an artifact of the lack of doubts of the government, or more generally, a consequence of having a government that is less pessimistic than the household. If we had a government that is more pessimistic than the household $\left(\sigma_{R}<\sigma_{A}<0\right)$, then paternalism and price manipulation will operate in the same direction: the government taxes more in good times, both because it considers them less probable (so the welfare loss from high taxes is considered small) and because it can make debt that is issued against good times cheaper. Obviously, we can consider also the other extreme of having only the government doubting the model and not the household $\left(\sigma_{R}<\sigma_{A}=0\right)$, which would eliminate completely the expectation management $\left(\tilde{\xi}_{t}=0\right)$ and allow only the paternalism effect in place. ${ }^{16}$

\footnotetext{
${ }^{15}$ Similarly to Karantounias (2013), we can employ the Marcet and Marimon (2019) methods in order to represent recursively the commitment problem, and interpret $\left(\Lambda_{t}, \tilde{\xi}_{t}\right)$ as state variables. Details are available upon request.

${ }^{16}$ Recall from the introduction that the case $\left(\sigma_{R}=0, \sigma_{A}<0\right)$ and the diametrically opposite case $\left(\sigma_{R}<\right.$ $\left.0, \sigma_{A}=0\right)$ are called respectively type 0 and type II ambiguity in the optimal policy nomenclature of Hansen
} 


\subsection{Small doubts about the model}

We sharpen our intuitive discussion of the previous section by considering the impacts of small doubts about the probability model of government spending. We express the relevant variables as function of the robustness parameters $\sigma \equiv\left(\sigma_{R}, \sigma_{A}\right)$ and perform a first-order Taylor expansion around the full confidence case of Lucas and Stokey $(1983), \sigma=(0,0) .{ }^{17}$

Proposition 5. ('Dynamics for small doubts about the model')

Let $x_{t}(\sigma)$ be shorthand for the endogenous variable $x_{t}\left(g^{t}, \sigma_{R}, \sigma_{A}\right)$, and let $x_{t}(0)$ denote the same variable evaluated at the full confidence economy, $\left(\sigma_{R}, \sigma_{A}\right)=(0,0)$.

- For small doubts about the model, the law of motion of the belief ratio (72) becomes

$$
\Lambda_{t}(\sigma)=\Lambda_{t-1}(\sigma)+\left(\sigma_{A}-\sigma_{R}\right)\left[V_{t}(0)-E_{t-1} V_{t}(0)\right], \Lambda_{0} \equiv 1
$$

- For small doubts about the model, the law of motion of $\tilde{\xi}_{t}$ (78) becomes

$$
\tilde{\xi}_{t}(\sigma)=\tilde{\xi}_{t-1}(\sigma)+\sigma_{A} \Phi(0)\left[U_{c t}(0) b_{t}(0)-E_{t-1} U_{c t}(0) b_{t}(0)\right], \tilde{\xi}_{0} \equiv 0
$$

Proof. See the Online Appendix.

Note that the martingales $\left(\Lambda_{t}, \tilde{\xi}_{t}\right)$ become random walks with respect to the reference model in the small doubts approximation. The increments in (80) and (81) depend on the respective utility, debt, and cost of distortionary taxation (as captured by $\Phi(0)$ ) of the Lucas and Stokey (1983) full-confidence economy. The approximate law (80) makes sharp the intuition we developed about paternalism. When there is a positive innovation in utility $\left(V_{t}(0)>E_{t-1} V_{t}(0)\right)$, the belief ratio increases (decreases) when the household doubts the model less (more) than the government $\left(\sigma_{A}>(<) \sigma_{R}\right)$. This comes from the fact that when the household doubts less than the government, it will assign relative more probability in good times than the government.

Consider now the quasi-linear utility function

$$
U(c, 1-h)=c-\frac{h^{1+\phi_{h}}}{1+\phi_{h}},
$$

and Sargent (2012).

${ }^{17}$ These heuristic expansions follow the logic of Holmes (1996) and Judd (1998) in perturbing around a known solution, which in the current environment is the Lucas and Stokey (1983) allocation. There are examples of similar in spirit expansions in terms of preference parameters in asset pricing and portfolio choice theory by Hansen et al. (2007) and Kogan and Uppal (2002). The Online Appendix provides a detailed analysis and caveats, and delves into the intricacies that are stemming from the fact that the coefficients in the Taylor expansion are random variables. 
which falls in the category of constant Frisch elasticity utility functions in proposition 4 for $\gamma=0, a_{h}=1$. In the Online Appendix we provide the analysis for more general utility functions but here we focus on the quasi-linear case, since it provides a setup where the government manipulates asset prices only through the worst-case beliefs of the household. The small-doubts approximation delivers illuminating formulas.

Proposition 6. ('Worst-case models for the quasi-linear case')

Assume the utility function (82) and let the reference model for $g_{t}$ have the Wold moving average representation

$$
g_{t}=\mu_{g}+\gamma(L) u_{t}^{g}
$$

where $\mu_{g}>0, \gamma(L) \equiv \sum_{i} \gamma_{i} L^{i}$ the lag polynomial, $\gamma(\beta)>0$ the present value of the polynomial coefficients, and $u_{t}^{g} \sim \operatorname{iid}\left(0, \sigma_{u}^{2}\right){ }^{18}$ Then,

1. The approximate dynamics of $\left(\Lambda_{t}, \tilde{\xi}_{t}\right)$ in (80) and (81) are determined by the innovation in the present value of $g_{t}$

$$
V_{t}(0)-E_{t-1} V_{t}(0)=b_{t}(0)-E_{t-1} b_{t}(0)=-\left(E_{t}-E_{t-1}\right)\left[\sum_{i=0}^{\infty} \beta^{i} g_{t+i}\right]=-\gamma(\beta) u_{t}^{g}
$$

2. The government's and the household's conditional likelihood ratios are approximately equal to

$$
n_{t}^{*}=1+\frac{1}{\theta_{R}} \gamma(\beta) u_{t}^{g}, \quad m_{t}^{*}=1+\frac{1}{\theta_{A}} \gamma(\beta) u_{t}^{g}
$$

3. The government's worst-case mean and variance of $u_{t}^{g}$ are approximately equal to

$$
E_{t} n_{t+1}^{*} u_{t+1}^{g}=\frac{1}{\theta_{R}} \gamma(\beta) \sigma_{u}^{2}>0, \quad \operatorname{Var}_{t}^{G o v \cdot}\left(u_{t+1}^{g}\right)=\sigma_{u}^{2}+\frac{\gamma(\beta)}{\theta_{R}} E_{t}\left(u_{t+1}^{g}\right)^{3}
$$

whereas the household's worst-case mean and variance can be described by the above formulas by replacing $\theta_{R}$ with $\theta_{A}$ and $n_{t+1}^{*}$ with $m_{t+1}^{*}$.

Proof. See the Online Appendix for the derivations. With quasi-linear utility, both the tax rate and labor become constant in the full-confidence case. This feature is behind the elegant expressions in (84).

\footnotetext{
${ }^{18}$ We drop here the restriction that $g$ lives on a countable space. We also assume that shocks have bounded support, so that $g$ remains positive.
} 
Proposition 6 connects the dynamics of paternalism and expectation management to innovations in government spending. The expression in (84) shows that a positive shock in government spending reduces utility in the full-confidence economy. Both the cautious government and the household assign higher probability mass on high spending shocks, as seen in (85). Consequently, the worst-case conditional means of $u_{t}^{g}$ are positive. In this example, the worst-case conditional variance remains unaltered, if we assume that the reference model has zero skewness, $E_{t}\left(u_{t+1}^{g}\right)^{3}=0$.

Consider now the implied tax and debt policies for the quasi-linear case. ${ }^{19}$

Proposition 7. ('Taxes and debt for the quasi-linear case')

Assume the utility function (82) and the reference process (83). Let $\tau \equiv \frac{\Phi(0) \phi_{h}}{1+\Phi(0)\left(1+\phi_{h}\right)}$ stand for the constant full-confidence tax rate, and let $h \equiv(1-\tau)^{1 / \phi_{h}}$ denote the respective full-confidence labor. ${ }^{20}$ We have the following results for small doubts about the model:

1. The tax rate, labor allocation and tax revenues $T_{t} \equiv \tau_{t} h_{t}$ follow random walks with respect to $\pi,{ }^{21}$

$$
\begin{aligned}
& \tau_{t}(\sigma)-\tau_{t-1}(\sigma)=\frac{\Phi(0) \phi_{h}}{\left(1+\Phi(0)\left(1+\phi_{h}\right)\right)^{2}} \underbrace{\left[\sigma_{A} \Phi(0)+\left(\sigma_{R}-\sigma_{A}\right)\right] \gamma(\beta) u_{t}^{g}}_{\text {Expectation management vs Paternalism }} \\
& h_{t}(\sigma)-h_{t-1}(\sigma)=-\frac{h}{1+\Phi(0)} \frac{\Phi(0)}{1+\Phi(0)\left(1+\phi_{h}\right)}\left[\sigma_{A} \Phi(0)+\left(\sigma_{R}-\sigma_{A}\right)\right] \gamma(\beta) u_{t}^{g} \\
& T_{t}(\sigma)-T_{t-1}(\sigma)=\frac{h}{1+\Phi(0)} \frac{\Phi(0) \phi_{h}}{\left(1+\Phi(0)\left(1+\phi_{h}\right)\right)^{2}}\left[\sigma_{A} \Phi(0)+\left(\sigma_{R}-\sigma_{A}\right)\right] \gamma(\beta) u_{t}^{g}
\end{aligned}
$$

2. The optimal debt policy is

$$
\begin{aligned}
b_{t}(\sigma)= & \underbrace{\frac{\tau h}{1-\beta}-E_{t} \sum_{i=0}^{\infty} \beta^{i} g_{t+i}}_{\text {Lucas and Stokey debt policy }} \\
& +\frac{(1-\beta)^{-1} h}{1+\Phi(0)} \frac{\Phi(0) \phi_{h}}{\left(1+\Phi(0)\left(1+\phi_{h}\right)\right)^{2}} \underbrace{\left[\sigma_{A} \Phi(0)+\left(\sigma_{R}-\sigma_{A}\right)\right] \gamma(\beta) \sum_{i=1}^{t} u_{i}^{g}}_{\text {Expectation management vs Paternalism }} .
\end{aligned}
$$

\footnotetext{
${ }^{19}$ See the Online Appendix for the small-doubts tax and debt policies for any utility function that satisfies the typical concavity and differentiability assumptions. Useful formulas for Markov shocks are also provided.

${ }^{20}$ The non-linear tax rate with doubts about the model is given by (74) for $\gamma=0$. From that same formula we get also the full-confidence tax rate by setting $\tilde{\xi}_{t}=0$ and $\Lambda_{t}=1$.

${ }^{21}$ The random walk result is obviously the manifestation of the martingale property in the small-doubts approximation and indicates the high persistence of optimal policy. These non-stationary results are not to be taken at face value for the long-run; we interpret them as being instructive for a short-run analysis starting at $t=0$. See the Online Appendix for further discussion.
} 
Proof. See the Online Appendix.

Proposition 7 sharpens our loose discussion in the previous section about the effects of expectation management and paternalism on the optimal tax rate and debt. Consider the tax rate in (87) and note how the innovation in $g_{t}$ is factored out, since it determines both the innovation in utility and the innovation in debt. If we eliminate paternalism, $\sigma_{R}=\sigma_{A}$, then the tax rate is clearly increasing when there is a reduction in government spending, increasing the price of debt (through a reduction of utility) sold against good times. Similarly, tax rates are reduced in bad times of high government spending.

Turning on paternalism, $\sigma_{R} \neq \sigma_{A}$, the reaction of the tax rate depends on the strength of expectation management versus the disagreement on the welfare cost of taxes. If the government is more pessimistic than the household $\left(\sigma_{R}<\sigma_{A}\right.$ or, equivalently, $\left.\sigma_{R} / \sigma_{A}>1\right)$, then the price manipulation through expectation management and the paternalistic efforts of the relatively pessimistic government act in the same direction. Thus, a positive spending shock leads to a reduction in the tax rate, as (87) shows. When the government doubts the model less than the household $\left(\sigma_{R}>\sigma_{A}\right)$, then the two effects act in the opposite direction and the reaction of the tax rate hinges on their relative strength. The size of the price manipulation effect depends on how much the government budget is relaxed by making debt cheaper, and therefore on the cost of distortionary taxation in the full-confidence economy, $\Phi(0)$. If $\Phi(0)>1-\sigma_{R} / \sigma_{A}$, the price manipulation though expectation management dominates, and the tax rate falls in bad times. ${ }^{22}$ If $\Phi(0)<1-\sigma_{R} / \sigma_{A}$ (which can only happen if $\sigma_{R} / \sigma_{A}<1$ since $\Phi(0)>0$ ), then the paternalism of the relatively optimistic government dominates, and the tax rate increases in bad times and falls in good times.

To conclude, optimal labor in (88) is the opposite image of the tax rate; when the tax rate increases, labor decreases. And optimal tax revenues in (89) reflect the behavior of the tax rate in (87). Similar comments hold for the optimal debt policy in (90). The Lucas and Stokey component reflects the fiscal hedging that the government conducts with state-contingent debt; the government is insuring against bad shocks by issuing less debt and issues more debt against good times. The second part reflects the price manipulation and the paternalism we highlighted in the analysis of the tax rate; higher taxes against a contingency are accompanied with higher debt issuance.

\section{Concluding remarks}

This paper provides a comprehensive overhaul of optimal policy problems where both the policymaker-leader and the agent-follower doubt the probability model of exogenous uncertainty.

\footnotetext{
${ }^{22} \mathrm{~A}$ sufficient condition for this outcome is obviously $\Phi(0)>1$.
} 
We have used a setup of a large firm that is facing a competitive fringe and a classic optimal taxation problem. The defining feature of this type of problems is the efforts of the leader to deal with her own model misspecification and her attempts to manage the endogenous cautious beliefs of the follower. Depending on the application there may be incentives to either amplify or mitigate the inherent pessimism of the follower.

It is useful to focus these remarks on future research avenues. We have not touched upon the issue of parameter uncertainty, but the methodology developed in the current paper would be useful in such an endeavor. Situations with multiple sources of uncertainty are particularly interesting. For example, Hansen and Sargent (2007) develop a machinery with two risk-sensitivity operators, that allow misspecification both within a model and across models. These ideas can lead to fragility of the worst-case beliefs, with intriguing consequences for the market price of risk, as Hansen and Sargent (2010) show. Optimal policy in such environments would add an additional channel of managing the endogenous fragile beliefs of the investor/household. ${ }^{23}$

Lastly, two more comments are due. The current study has focused on a situation where multiple agents perturb a baseline probability model by surrounding it with a set of unstructured models, and studied the implications for optimal policy design. More elaborate schemes of both structured and unstructured ambiguity can be constructed, following the lead of Hansen and Sargent (2020). Furthermore, by representing ambiguity aversion with smooth preferences, the current paper focused on how small changes in the instrument of the policy maker lead to small changes in the worst-case beliefs of the follower. If we followed other approaches (non-smooth or non-additive) to represent ambiguity aversion, regions of inaction or inertia could potentially appear, as in the seminal work of Dow and da Costa Werlang (1992). Inertial behavior would require large actions from the policymaker to induce a change in the pessimistic beliefs of the follower. Such directions are all worthy of future research.

\footnotetext{
${ }^{23}$ See Ju and Miao (2012) and Collard et al. (2018) for further applications of learning under ambiguity ideas in asset pricing.
} 


\section{A Monopolist and competitive fringe}

\section{A.1 Recursive formulation}

We follow here the method of Kydland and Prescott (1980), which relies on finding the proper pseudo-state variables that capture the forward-looking constraints. We formulate the problem before the realization of uncertainty.

Let $P_{t} \equiv \frac{1}{\sigma_{A}} \ln E_{t} \exp \left(\sigma_{A} V_{t+1}\right)$ denote the risk-sensitive "expected" profits of the competitive fringe and recall that $V_{t}$ follows recursion (13). The respective recursion in terms of $P_{t}$ becomes

$$
P_{t-1}=\frac{1}{\sigma_{A}} \ln E_{t-1} \exp \left(\sigma_{A}\left(\Pi_{t}^{F}+\beta P_{t}\right)\right)
$$

The monopolist's present value of profits in (17) can be represented recursively as

$$
W_{t}=\Pi_{t}^{M}+\beta \min _{n_{t+1} \geq 0, E_{t} n_{t+1}=1}\left\{E_{t} n_{t+1} W_{t+1}+\theta_{R} \varepsilon_{t}\left(n_{t+1}\right)\right\} .
$$

Let

$$
J_{t} \equiv \min _{n_{t+1} \geq 0, E_{t} n_{t+1}=1} E_{t} n_{t+1} W_{t+1}+\theta_{R} \varepsilon_{t}\left(n_{t+1}\right)
$$

denote the "expected" profits of the monopolist under the worst-case beliefs (taking into account also the entropy penalty). Using this definition, we get that $W_{t}=\Pi_{t}^{M}+\beta J_{t}$. Thus, we can rewrite the profit recursion (A.2) in terms of $J_{t}$,

$$
J_{t-1}=\min _{n_{t} \geq 0, E_{t-1} n_{t}=1} E_{t-1} n_{t}\left[\Pi_{t}^{M}+\beta J_{t}\right]+\theta_{R} \varepsilon_{t-1}\left(n_{t}\right)
$$

Bellman equation. Let $J(\cdot)$ denote the value function for the commitment problem from period one onward as a function of the state $\left(Q, \bar{q}, v_{-}, i_{-}, P_{-}\right)$. Underscore "-" denotes previous period. The state includes the two endogenous backward-looking variables $Q, \bar{q}$, the exogenous shock $v_{-}$, and $\left(i_{-}, P_{-}\right)$, which capture respectively the promises of monopolist to a particular evolution of the fringe's output and profits. The value function takes the form ${ }^{24}$

$$
\begin{aligned}
& J\left(Q, \bar{q}, v_{-}, i_{-}, P_{-}\right)=\max _{\left\{u(\epsilon), i(\epsilon), m^{*}(\epsilon), P(\epsilon)\right\}} \min _{n(\epsilon) \geq 0} \int n(\epsilon)\left\{\left[A_{0}-A_{1}(Q+\bar{q})+\left(\rho v_{-}+C_{\epsilon} \epsilon\right)\right] Q\right. \\
& \left.-\left(e Q+\frac{1}{2} g Q^{2}+\frac{1}{2} c u^{2}(\epsilon)\right)+\beta J\left(Q^{\prime}, \bar{q}^{\prime}, \rho v_{-}+C_{\epsilon} \epsilon, i(\epsilon), P(\epsilon)\right)\right\} f(\epsilon) d \epsilon+\theta_{R} \int n(\epsilon) \ln n(\epsilon) f(\epsilon) d \epsilon
\end{aligned}
$$

\footnotetext{
${ }^{24}$ Notation like $i(\epsilon)$ or $P(\epsilon)$ indicates that the control variables are functions of the shock innovation $\epsilon$.
} 
subject to

$$
\begin{aligned}
Q^{\prime}= & Q+u(\epsilon) \\
\bar{q}^{\prime}= & \bar{q}+i(\epsilon) \\
i_{-}= & \beta c^{-1} \int m^{*}(\epsilon)\left[\left(A_{0}-A_{1}(Q+\bar{q})+\rho v_{-}+C_{\epsilon} \epsilon\right)-(d+h \bar{q})\right] f(\epsilon) d \epsilon \\
& +\beta \int m^{*}(\epsilon) i(\epsilon) f(\epsilon) d \epsilon \\
m^{*}(\epsilon)= & \frac{\exp \left(\sigma_{A}\left(\Pi^{F}(\epsilon)+\beta P(\epsilon)\right)\right)}{\int \exp \left(\sigma_{A}\left(\Pi^{F}(\epsilon)+\beta P(\epsilon)\right)\right) f(\epsilon) d \epsilon} \\
P_{-}= & \frac{1}{\sigma_{A}} \ln \int \exp \left(\sigma_{A}\left(\Pi^{F}(\epsilon)+\beta P(\epsilon)\right)\right) f(\epsilon) d \epsilon \\
\int n(\epsilon) f(\epsilon) d \epsilon= & 1
\end{aligned}
$$

where $\Pi^{F}(\epsilon)$ is shorthand for the fringe's period equilibrium profits,

$$
\Pi^{F}(\epsilon) \equiv\left(A_{0}-A_{1}(Q+\bar{q})+\rho v_{-}+C_{\epsilon} \epsilon\right) \bar{q}-\left(d \bar{q}+\frac{1}{2} h \bar{q}^{2}+\frac{1}{2} c i^{2}(\epsilon)\right)
$$

Initial period problem. The state variables $i$ and $P$ are pseudo-state variables, that is, they are useful to capture the promises of the monopolist to the fringe and, as a result, to represent the commitment problem recursively. Their initial values $\left(i_{0}, P_{0}\right)$ are chosen optimally at $t=0$, when there are no promises to be kept. Given $\left(Q_{0}, \bar{q}_{0}, v_{0}\right)$, the initial period problem is to choose $\left(u_{0}, i_{0}, P_{0}\right)$ to maximize

$$
\left(A_{0}-A_{1}\left(Q_{0}+\bar{q}_{0}\right)+v_{0}\right) Q_{0}-\left(e Q_{0}+\frac{1}{2} g Q_{0}^{2}+\frac{1}{2} c u_{0}^{2}\right)+\beta J\left(Q_{0}+u_{0}, \bar{q}_{0}+i_{0}, v_{0}, i_{0}, P_{0}\right) .
$$

\section{A.2 Lagrangians}

The optimality conditions of the monopolist's problem are listed and analyzed in the text. For completeness, I provide here the respective Lagrangians. All multipliers dated at $t$ are functions of information at $t$ and are scaled by $\beta^{t}$ and the reference joint density of the partial history of shocks $\epsilon^{t}$.

\section{A.2.1 Adjustment costs, $c>0$}

The Lagrangian of problem 2 is as follows. 


$$
\begin{aligned}
L= & E_{0} \sum_{t=0}^{\infty} \beta^{t} N_{t}\left[\left(A_{0}-A_{1}\left(Q_{t}+\bar{q}_{t}\right)+v_{t}\right) Q_{t}-\left(e Q_{t}+\frac{1}{2} g Q_{t}^{2}+\frac{1}{2} c u_{t}^{2}\right)\right]+\beta \theta_{R} E_{0} \sum_{t=0}^{\infty} \beta^{t} N_{t} \varepsilon_{t}\left(n_{t+1}\right) \\
& -E_{0} \sum_{t=0}^{\infty} \beta^{t} \lambda_{t}^{Q}\left[Q_{t+1}-Q_{t}-u_{t}\right]-E_{t} \sum_{t=0}^{\infty} \beta^{t} \lambda_{t}^{\bar{q}}\left[\bar{q}_{t+1}-\bar{q}_{t}-i_{t}\right] \\
& -E_{0} \sum_{t=0}^{\infty} \beta^{t} \xi_{t}^{i}\left[i_{t}-\beta c^{-1}\left[A_{0}-A_{1}\left(Q_{t+1}+\bar{q}_{t+1}\right)-\left(d+h \bar{q}_{t+1}\right)+\rho v_{t}+C_{\epsilon} E_{t} m_{t+1}^{*} \epsilon_{t+1}\right]-\beta E_{t} m_{t+1}^{*} i_{t+1}\right] \\
& -E_{0} \sum_{t=0}^{\infty} \beta^{t} \beta E_{t} \mu_{t+1}\left[m_{t+1}^{*}-\frac{\exp \left(\sigma_{A} V_{t+1}\right)}{E_{t} \exp \left(\sigma_{A} V_{t+1}\right)}\right] \\
& -E_{0} \sum_{t=0}^{\infty} \beta^{t} \xi_{t}^{V}\left[V_{t}-\left[\left(A_{0}-A_{1}\left(Q_{t}+\bar{q}_{t}\right)+v_{t}\right) \bar{q}_{t}-\left(d \bar{q}_{t}+\frac{1}{2} h \bar{q}_{t}^{2}+\frac{1}{2} c i_{t}^{2}\right)\right]-\frac{\beta}{\sigma_{A}} \ln E_{t} \exp \left(\sigma_{A} V_{t+1}\right)\right] \\
& -E_{0} \sum_{t=0}^{\infty} \beta^{t}\left[\beta E_{t} \nu_{t+1}^{N}\left[N_{t+1}-n_{t+1} N_{t}\right]+\nu_{t}^{n}\left(E_{t} n_{t+1}-1\right)\right]
\end{aligned}
$$

\section{A.2.2 No adjustment costs}

Consider now the Lagrangian without adjustment costs, $c=0$. In this case we do not need to keep track of the laws of motion (18) and (19). Moreover, the optimality condition of the fringe (20) is simplified.

$$
\begin{aligned}
L= & E_{0} \sum_{t=0}^{\infty} \beta^{t} N_{t}\left[\left(A_{0}-A_{1}\left(Q_{t}+\bar{q}_{t}\right)+v_{t}\right) Q_{t}-\left(e Q_{t}+\frac{1}{2} g Q_{t}^{2}\right)\right]+\beta \theta_{R} E_{0} \sum_{t=0}^{\infty} \beta^{t} N_{t} \varepsilon_{t}\left(n_{t+1}\right) \\
& +E_{0} \sum_{t=0}^{\infty} \beta^{t+1} \xi_{t}^{i}\left[A_{0}-A_{1}\left(Q_{t+1}+\bar{q}_{t+1}\right)-\left(d+h \bar{q}_{t+1}\right)+\rho v_{t}+C_{\epsilon} E_{t} m_{t+1}^{*} \epsilon_{t+1}\right] \\
& -E_{0} \sum_{t=0}^{\infty} \beta^{t} \beta E_{t} \mu_{t+1}\left[m_{t+1}^{*}-\frac{\exp \left(\sigma_{A} V_{t+1}\right)}{E_{t} \exp \left(\sigma_{A} V_{t+1}\right)}\right] \\
& -E_{0} \sum_{t=0}^{\infty} \beta^{t} \xi_{t}^{V}\left[V_{t}-\left[\left(A_{0}-A_{1}\left(Q_{t}+\bar{q}_{t}\right)+v_{t}\right) \bar{q}_{t}-\left(d \bar{q}_{t}+\frac{1}{2} h \bar{q}_{t}^{2}\right)\right]-\frac{\beta}{\sigma_{A}} \ln E_{t} \exp \left(\sigma_{A} V_{t+1}\right)\right] \\
& -E_{0} \sum_{t=0}^{\infty} \beta^{t}\left[\beta E_{t} \nu_{t+1}^{N}\left[N_{t+1}-n_{t+1} N_{t}\right]+\nu_{t}^{n}\left(E_{t} n_{t+1}-1\right)\right]
\end{aligned}
$$

\section{A.3 Proof of proposition 1}

Proof. The Lagrangian is stated in (A.12). Consider first the optimality condition with respect to the fringe's profits $V_{t}$. This takes the form 


$$
\xi_{t}^{V}=\sigma_{A} m_{t}^{*}\left[\mu_{t}-E_{t-1} m_{t}^{*} \mu_{t}\right]+m_{t}^{*} \xi_{t-1}^{V}, \xi_{0}^{V} \equiv 0
$$

But from the optimality condition with respect to the worst-case likelihood ratio $m_{t+1}^{*}$ we get $\mu_{t+1}=C_{\epsilon} \epsilon_{t+1} \xi_{t}^{i}$. Use this result to replace $\mu_{t}$ in the law of motion of $\xi_{t}^{V}$ and recall the definition of the normalized multipliers to get (43). Note that, as in the case of adjustment costs, we have a martingale result with respect to the monopolist's measure, $E_{t} n_{t+1}^{*} \tilde{\xi}_{t+1}^{V}=\tilde{\xi}_{t}^{V}$.

The optimality condition with respect to $Q_{t+1}$, after normalizing with $N_{t}^{*}$, takes the form

$$
E_{t} n_{t+1}^{*}\left[p_{t+1}-A_{1} Q_{t+1}-\left(e+g Q_{t+1}\right)\right]-A_{1}\left[\tilde{\xi}_{t}^{i}+\bar{q}_{t+1} E_{t} n_{t+1}^{*} \tilde{\xi}_{t+1}^{V}\right]=0
$$

Use the martingale result for $\tilde{\xi}_{t}^{V}$ to get (40). Optimality condition (41) is just the standard first-order condition with respect to $\bar{q}_{t+1}$, using again the normalized variables. To get (44) use the fact that $E_{t} m_{t+1}^{*} p_{t+1}=d+\bar{h} q_{t+1}$ and the martingale result for $\tilde{\xi}_{t}^{V}$ to rewrite (41) as

$$
-A_{1}\left[Q_{t+1}+\tilde{\xi}_{t}^{V} \bar{q}_{t+1}\right]-\left(A_{1}+h\right) \tilde{\xi}_{t}^{i}+E_{t} n_{t+1}^{*} \tilde{\xi}_{t+1}^{V}\left(p_{t+1}-E_{t} m_{t+1}^{*} p_{t+1}\right)=0
$$

Consider now the last term in the left-hand side above. We have

$$
\begin{aligned}
E_{t} n_{t+1}^{*} \tilde{\xi}_{t+1}^{V}\left(p_{t+1}-E_{t} m_{t+1}^{*} p_{t+1}\right) \stackrel{(43)}{=} & \sigma_{A} C_{\epsilon} E_{t} m_{t+1}^{*}\left(\epsilon_{t+1}-E_{t} m_{t+1}^{*} \epsilon_{t+1}\right)\left(p_{t+1}-E_{t} m_{t+1}^{*} p_{t+1}\right) \tilde{\xi}_{t}^{i} \\
& +\tilde{\xi}_{t}^{V} E_{t} m_{t+1}^{*}\left(p_{t+1}-E_{t} m_{t+1}^{*} p_{t+1}\right) \\
= & \sigma_{A} C_{\epsilon}^{2}\left(E_{t} m_{t+1}^{*}\left(\epsilon_{t+1}-E_{t} m_{t+1}^{*} \epsilon_{t+1}\right)^{2}\right) \tilde{\xi}_{t}^{i}=\sigma_{A} C_{\epsilon}^{2} \operatorname{Var}_{t}^{F}\left(\epsilon_{t+1}\right) \tilde{\xi}_{t}^{i},
\end{aligned}
$$

where I used the fact that the innovation in prices is $p_{t+1}-E_{t} m_{t+1}^{*} p_{t+1}=C_{\epsilon}\left(\epsilon_{t+1}-E_{t} m_{t+1}^{*} \epsilon_{t+1}\right)$. Use this result in (A.13), collect terms that multiply $\tilde{\xi}_{t}^{i}$ and rearrange to get (44).

\section{A.4 Optimal quantities}

\section{A.4.1 Preliminaries}

We show here how to find $\left(Q_{t+1}, \bar{q}_{t+1}\right)$ as functions of the exogenous state $v_{t}$, the multiplier $\tilde{\xi}_{t}^{V}$, and the implied worst-case first and second moments of the monopolist and the fringe.

Use (44) to replace $\tilde{\xi}_{t}^{i}$ in (40). This delivers an optimality condition for the monopolist in terms of $\left(Q_{t+1}, \bar{q}_{t+1}\right)$, the state variables $\left(\tilde{\xi}_{t}^{V}, v_{t}\right)$ and the worst-case moments of the monopolist and the fringe. Use the resulting equation and the reaction function of the fringe (15) to get the 
system

$$
\mathbf{A}_{\mathbf{t}} \cdot\left(\begin{array}{c}
Q_{t+1} \\
\bar{q}_{t+1}
\end{array}\right)=\mathbf{B}_{\mathbf{t}} \cdot\left(\begin{array}{c}
1 \\
v_{t}
\end{array}\right) \Rightarrow\left(\begin{array}{c}
Q_{t+1} \\
\bar{q}_{t+1}
\end{array}\right)=\mathbf{A}_{\mathbf{t}}^{-1} \mathbf{B}_{\mathbf{t}} \cdot\left(\begin{array}{c}
1 \\
v_{t}
\end{array}\right)
$$

where

$$
\begin{aligned}
& \mathbf{A}_{\mathbf{t}} \equiv\left[\begin{array}{cc}
A_{1}\left(2-\omega_{t}\right)+g & A_{1}\left(1+\left(1-\omega_{t}\right) \tilde{\xi}_{t}^{V}\right) \\
A_{1} & A_{1}+h
\end{array}\right], \omega_{t} \equiv \frac{A_{1}}{A_{1}+h-\sigma_{A} C_{\epsilon}^{2} \operatorname{Var}_{t}^{F}\left(\epsilon_{t+1}\right)} \\
& \mathbf{B}_{\mathbf{t}} \equiv\left[\begin{array}{cc}
A_{0}-e+C_{\epsilon} E_{t} n_{t+1}^{*} \epsilon_{t+1} & \rho \\
A_{0}-d+C_{\epsilon} E_{t} m_{t+1}^{*} \epsilon_{t+1} & \rho
\end{array}\right]
\end{aligned}
$$

Note that matrices $\left(\mathbf{A}_{\mathbf{t}}, \mathbf{B}_{\mathbf{t}}\right)$ depend on the value of the multiplier $\tilde{\xi}_{t}^{V}$ and on the worst-case first $\left(E_{t} n_{t+1}^{*} \epsilon_{t+1}, E_{t} m_{t+1}^{*} \epsilon_{t+1}\right)$ and second moments $\left(\operatorname{Var}_{t}^{F}\left(\epsilon_{t+1}\right)\right)$. Thus, if we knew the worstcase likelihood ratios $n_{t+1}^{*}$ and $m_{t+1}^{*}$, we could solve for the optimal choice of quantities as a (non-linear) function of $\left(\tilde{\xi}_{t}^{V}, v_{t}\right)$. This feature of the solution will be useful for the proof of proposition 3 and for the solution algorithm in the next section.

\section{A.4.2 Proof of proposition 3}

Proof. The inverse of $\mathbf{A}_{\mathbf{t}}$ in (A.15) is

$$
\begin{aligned}
\mathbf{A}_{\mathbf{t}}^{-\mathbf{1}} & =\frac{1}{\Xi_{t}}\left[\begin{array}{cc}
1 & -\varsigma\left(1+\left(1-\omega_{t}\right) \tilde{\xi}_{t}^{V}\right) \\
-\varsigma & \varsigma\left(2-\omega_{t}+g / A_{1}\right)
\end{array}\right] \\
\Xi_{t} & \equiv A_{1}\left(2-\omega_{t}+g / A_{1}-\varsigma\left(1+\left(1-\omega_{t}\right) \tilde{\xi}_{t}^{V}\right)\right), \quad \varsigma \equiv \frac{A_{1}}{A_{1}+h}
\end{aligned}
$$

Note that when $\sigma_{A}=0$ we have $\omega_{t}=\varsigma$. Turning to the solution of the system, we get

$$
\mathbf{A}_{\mathbf{t}}{ }^{-1} \mathbf{B}_{\mathbf{t}}=\frac{1}{\Xi_{t}}\left[\begin{array}{cc}
I_{11, t} & I_{12, t} \\
I_{21, t} & I_{22, t}
\end{array}\right]
$$

where 


$$
\begin{aligned}
I_{11, t} & \equiv A_{0}-e+C_{\epsilon} E_{t} n_{t+1}^{*} \epsilon_{t+1}-\varsigma\left(1+\left(1-\omega_{t}\right) \tilde{\xi}_{t}^{V}\right)\left[A_{0}-d+C_{\epsilon} E_{t} m_{t+1}^{*} \epsilon_{t+1}\right] \\
I_{12, t} & \equiv \rho\left(1-\varsigma\left(1+\left(1-\omega_{t}\right) \tilde{\xi}_{t}^{V}\right)\right) \\
I_{21, t} & \equiv \varsigma\left[-\left(A_{0}-e+C_{\epsilon} E_{t} n_{t+1}^{*} \epsilon_{t+1}+\left(2-\omega_{t}+g / A_{1}\right)\left(A_{0}-d+C_{\epsilon} E_{t} m_{t+1}^{*} \epsilon_{t+1}\right)\right]\right. \\
I_{22, t} & \equiv \rho \varsigma\left(1-\omega_{t}+g / A_{1}\right)
\end{aligned}
$$

We will take now derivatives ignoring the dependence of the endogenous worst-case moments $\left(E_{t} n_{t+1}^{*} \epsilon_{t+1}, E_{t} m_{t+1}^{*} \epsilon_{t+1}\right)$ and $\operatorname{Var}_{t}^{F}\left(\epsilon_{t+1}\right)$ (which affects $\left.\omega_{t}\right)$ on $\tilde{\xi}_{t}^{V}$. The respective derivatives are

$$
\begin{aligned}
\frac{\partial \Xi_{t}}{\partial \tilde{\xi}_{t}^{V}} & =-\varsigma A_{1}\left(1-\omega_{t}\right) \\
\frac{\partial I_{11, t}}{\partial \tilde{\xi}_{t}^{V}} & =-\varsigma\left(1-\omega_{t}\right)\left[A_{0}-d+C_{\epsilon} E_{t} m_{t+1}^{*} \epsilon_{t+1}\right] \\
\frac{\partial I_{12, t}}{\partial \tilde{\xi}_{t}^{V}} & =-\rho \varsigma\left(1-\omega_{t}\right) \\
\frac{\partial I_{21, t}}{\partial \tilde{\xi}_{t}^{V}} & =0 \\
\frac{\partial I_{22, t}}{\partial \tilde{\xi}_{t}^{V}} & =0
\end{aligned}
$$

Turning now to the solution, we have

$$
\begin{aligned}
Q_{t+1} & =\frac{1}{\Xi_{t}}\left[I_{11, t}+I_{12, t} v_{t}\right] \\
\bar{q}_{t+1} & =\frac{1}{\Xi_{t}}\left[I_{21, t}+I_{22, t} v_{t}\right]
\end{aligned}
$$

Therefore,

$$
\begin{aligned}
& \left.\frac{\partial Q_{t+1}}{\partial \tilde{\xi}_{t}^{V}}\right|_{\text {const. }} \quad=\quad-\Xi_{t}^{-2} \frac{\partial \Xi_{t}}{\partial \tilde{\xi}_{t}^{V}}\left(I_{11, t}+I_{12, t}\right)+\Xi_{t}^{-1}\left[\frac{\partial I_{11, t}}{\partial \tilde{\xi}_{t}^{V}}+\frac{\partial I_{12, t}}{\partial \tilde{\xi}_{t}^{V}} v_{t}\right] \\
& =\quad \Xi_{t}^{-1}\left[-Q_{t+1} \frac{\partial \Xi_{t}}{\partial \tilde{\xi}_{t}^{V}}+\frac{\partial I_{11, t}}{\partial \tilde{\xi}_{t}^{V}}+\frac{\partial I_{12, t}}{\partial \tilde{\xi}_{t}^{V}} v_{t}\right] \\
& \stackrel{(A .23)-(A .25)}{=}-\varsigma\left(1-\omega_{t}\right) \Xi_{t}^{-1}\left[A_{0}-d+\rho v_{t}+C_{\epsilon} E_{t} m_{t+1}^{*} \epsilon_{t+1}-A_{1} Q_{t+1}\right] \\
& =-\varsigma\left(1-\omega_{t}\right) \Xi_{t}^{-1}\left[E_{t} m_{t+1}^{*} p_{t+1}+A_{1} \bar{q}_{t+1}-d\right]
\end{aligned}
$$

Recall though that $E_{t} m_{t+1}^{*} p_{t+1}=d+h \bar{q}_{t+1}$. Thus, 


$$
\left.\frac{\partial Q_{t+1}}{\partial \tilde{\xi}_{t}^{V}}\right|_{\text {const. }}=-\varsigma\left(1-\omega_{t}\right) \Xi_{t}^{-1}\left(A_{1}+h\right) \bar{q}_{t+1}<0
$$

Proceeding to the derivative of $\bar{q}_{t+1}$ and using (A.23) and (A.26)-(A.27) we have

$$
\left.\frac{\partial \bar{q}_{t+1}}{\partial \tilde{\xi}_{t}^{V}}\right|_{\text {const. }}=-\Xi_{t}^{-1} \frac{\partial \Xi_{t}}{\partial \tilde{\xi}_{t}^{V}} \bar{q}_{t+1}=\varsigma\left(1-\omega_{t}\right) \Xi_{t}^{-1} A_{1} \bar{q}_{t+1}>0
$$

Finally, using (A.30) and (A.31), we get

$$
\left.\frac{\partial Q_{t+1}^{\text {total }}}{\partial \tilde{\xi}_{t}^{V}}\right|_{\text {const. }}=\left.\frac{\partial Q_{t+1}}{\partial \tilde{\xi}_{t}^{V}}\right|_{\text {const. }}+\left.\frac{\partial \bar{q}_{t+1}}{\partial \tilde{\xi}_{t}^{V}}\right|_{\text {const. }}=-\varsigma\left(1-\omega_{t}\right) \Xi_{t}^{-1} h \bar{q}_{t+1}<0
$$

\section{A.5 Illustration details}

Given the uncertainty and timing assumptions, we have the following inverse demand functions:

$$
\begin{aligned}
p_{0} & =A_{0}-A_{1}\left(Q_{0}+\bar{q}_{0}\right) \\
p_{1}\left(\epsilon_{1}\right) & =A_{0}-A_{1}\left(Q_{1}+\bar{q}_{1}\right)+C_{\epsilon} \epsilon_{1}, \forall \epsilon_{1} \\
p_{2}\left(\epsilon_{1}\right) & =A_{0}-A_{1}\left(Q_{2}\left(\epsilon_{1}\right)+\bar{q}_{2}\left(\epsilon_{1}\right)\right), \forall \epsilon_{1} .
\end{aligned}
$$

Profits and worst-case beliefs. The period profits of the monopolist for $t=1,2$ are

$$
\begin{aligned}
& \Pi_{1}^{M}\left(\epsilon_{1}\right)=p_{1}\left(\epsilon_{1}\right) Q_{1}-\left(e Q_{1}+\frac{1}{2} g Q_{1}^{2}\right), \forall \epsilon_{1} \\
& \Pi_{2}^{M}\left(\epsilon_{1}\right)=p_{2}\left(\epsilon_{1}\right) Q_{2}\left(\epsilon_{1}\right)-\left(e Q_{2}\left(\epsilon_{1}\right)+\frac{1}{2} g Q_{2}\left(\epsilon_{1}\right)^{2}\right), \forall \epsilon_{1}
\end{aligned}
$$

The period profits of the competitive fringe are

$$
\begin{aligned}
& \Pi_{1}^{F}\left(\epsilon_{1}\right)=p_{1}\left(\epsilon_{1}\right) \bar{q}_{1}-\left(d \bar{q}_{1}+\frac{1}{2} h \bar{q}_{1}^{2}\right), \forall \epsilon_{1} \\
& \Pi_{2}^{F}\left(\epsilon_{1}\right)=p_{2}\left(\epsilon_{1}\right) \bar{q}_{2}\left(\epsilon_{1}\right)-\left(d \bar{q}_{2}\left(\epsilon_{1}\right)+\frac{1}{2} h \bar{q}_{2}\left(\epsilon_{1}\right)^{2}\right), \forall \epsilon_{1}
\end{aligned}
$$


The worst-case beliefs of the monopolist and the competitive fringe are

$$
n_{1}^{*}\left(\epsilon_{1}\right)=\frac{\exp \left(\sigma_{R} W_{1}\left(\epsilon_{1}\right)\right)}{E \exp \left(\sigma_{R} W_{1}\right)} \quad \text { and } \quad m_{1}^{*}\left(\epsilon_{1}\right)=\frac{\exp \left(\sigma_{A} V_{1}\left(\epsilon_{1}\right)\right)}{E \exp \left(\sigma_{A} V_{1}\right)}
$$

where

$$
\begin{aligned}
W_{1}\left(\epsilon_{1}\right) & =\Pi_{1}^{M}\left(\epsilon_{1}\right)+\beta \Pi_{2}^{M}\left(\epsilon_{1}\right), \forall \epsilon_{1} \\
V_{1}\left(\epsilon_{1}\right) & =\Pi_{1}^{F}\left(\epsilon_{1}\right)+\beta \Pi_{2}^{F}\left(\epsilon_{1}\right), \forall \epsilon_{1} .
\end{aligned}
$$

Multipliers. Consider now the shadow value of the fringe's forecast, $\tilde{\xi}_{t}^{i}$. We have

$$
\begin{aligned}
\tilde{\xi}_{0}^{i} & =-\frac{A_{1}}{A_{1}+h-\sigma_{A} C_{\epsilon}^{2} \operatorname{Var}^{F}\left(\epsilon_{1}\right)} Q_{1}<0 \\
\tilde{\xi}_{1}^{i}\left(\epsilon_{1}\right) & =-\frac{A_{1}}{A_{1}+h}\left[Q_{2}\left(\epsilon_{1}\right)+\tilde{\xi}_{1}^{V}\left(\epsilon_{1}\right) \bar{q}_{2}\left(\epsilon_{1}\right)\right], \forall \epsilon_{1}
\end{aligned}
$$

Furthermore, $\tilde{\xi}_{0}^{V}=0$ and

$$
\tilde{\xi}_{1}^{V}\left(\epsilon_{1}\right)=\sigma_{A} \frac{m_{1}^{*}\left(\epsilon_{1}\right)}{n_{1}^{*}\left(\epsilon_{1}\right)} C_{\epsilon}\left[\epsilon_{1}-E m_{1}^{*} \epsilon_{1}\right] \tilde{\xi}_{0}^{i}
$$

Note that since $\tilde{\xi}_{0}^{i}<0$, the value of the multiplier is positive for "good" shocks $\left(\epsilon_{1}-E m_{1}^{*} \epsilon_{1}>\right.$ $0)$ and negative for "bad" shocks, $\left(\epsilon_{1}-E m_{1}^{*} \epsilon_{1}<0\right)$. Thus, the monopolist has an incentive to increase the profits of the fringe in good times, and decrease the profits of the fringe in bad times.

Matrices. In this three period example we have no persistence, so there is no need to keep track of $v_{t}$. Let $C_{t}$ denote the $2 \times 1$ vector that is the product of the matrix $\mathbf{B}_{\mathbf{t}}$ and $\left(1, v_{t}\right)^{\prime}$ in the right-hand side of (A.14). For our example $C_{t}$ takes the form,

$$
C_{0} \equiv\left(\begin{array}{c}
A_{0}-e+C_{\epsilon} E n_{1}^{*} \epsilon_{1} \\
A_{0}-d+C_{\epsilon} E m_{1}^{*} \epsilon_{1}
\end{array}\right), \quad C_{1} \equiv\left(\begin{array}{c}
A_{0}-e \\
A_{0}-d
\end{array}\right)
$$

Furthermore, the matrix $\mathbf{A}_{\mathbf{t}}$ in (A.15) becomes 


$$
\begin{aligned}
\mathbf{A}_{\mathbf{0}} & =\left[\begin{array}{cc}
2 A_{1}+g-\frac{A_{1}^{2}}{A_{1}+h-\sigma_{A} C_{\epsilon}^{2} \operatorname{Var}^{F}\left(\epsilon_{1}\right)} & A_{1} \\
A_{1} & A_{1}+h
\end{array}\right] \\
\mathbf{A}_{\mathbf{1}}\left(\epsilon_{1}\right)= & {\left[\begin{array}{cc}
2 A_{1}+g-\frac{A_{1}^{2}}{A_{1}+h} & A_{1}\left[1+\frac{h}{A_{1}+h} \tilde{\xi}_{1}^{V}\left(\epsilon_{1}\right)\right] \\
A_{1} & A_{1}+h
\end{array}\right] . }
\end{aligned}
$$

Algorithm. Make an initial guess for $\left.\left(n_{1}^{*}\left(\epsilon_{1}\right), m_{1}^{*}\left(\epsilon_{1}\right)\right)\right)$ and consider the following procedure:

- Given $\left(n_{1}^{*}\left(\epsilon_{1}\right), m_{1}^{*}\left(\epsilon_{1}\right)\right)$, determine the respective worst-case moments, and calculate $C_{0}$ and $\mathbf{A}_{\mathbf{0}}$ from (A.39) and (A.40). Solve for $\left(Q_{1}, \bar{q}_{1}\right)$

$$
\left(\begin{array}{c}
Q_{1} \\
\bar{q}_{1}
\end{array}\right)=\mathbf{A}_{\mathbf{0}}{ }^{-1} C_{0} .
$$

- Use the solution to calculate multiplier $\tilde{\xi}_{0}^{i}$ and multipliers $\tilde{\xi}_{1}^{V}\left(\epsilon_{1}\right)$ (for each $\epsilon_{1}$ ) from (A.36) and (A.38) respectively.

- Given $\tilde{\xi}_{1}^{V}\left(\epsilon_{1}\right)$ form $\mathbf{A}_{\mathbf{1}}\left(\epsilon_{1}\right)$ from (A.41) and solve for $\left(Q_{2}\left(\epsilon_{1}\right), \bar{q}_{2}\left(\epsilon_{1}\right)\right)$ as

$$
\left(\begin{array}{c}
Q_{2}\left(\epsilon_{1}\right) \\
\bar{q}_{2}\left(\epsilon_{1}\right)
\end{array}\right)=\mathbf{A}_{\mathbf{1}}\left(\epsilon_{1}\right)^{-1} C_{1}, \forall \epsilon_{1} .
$$

- Given the solution for quantities, calculate the profits of the monopolist and the fringe using (A.34) and (A.35), update the worst-case likelihood ratios $\left(n_{1}^{*}\left(\epsilon_{1}\right), m_{1}^{*}\left(\epsilon_{1}\right)\right)$ from (A.33) and go back to the initial step.

- Iterate till convergence.

We can use unity as an initial guess for the likelihood ratios, $n_{1}^{*}\left(\epsilon_{1}\right)=m_{1}^{*}\left(\epsilon_{1}\right)=1, \forall \epsilon_{1}$.

Full confidence in the model. Assume that the fringe is not doubting the model, $\sigma_{A}=0$. Then the matrix $\mathbf{A}_{\mathbf{t}}$ becomes

$$
\mathbf{A}_{\mathbf{t}}=\mathbf{A} \equiv\left[\begin{array}{cc}
2 A_{1}+g-\frac{A_{1}^{2}}{A_{1}+h} & A_{1} \\
A_{1} & A_{1}+h
\end{array}\right], t=1,2
$$

If the monopolist has also full confidence in the model $\left(\sigma_{R}=0\right)$, then 


$$
\left(\begin{array}{c}
Q_{1} \\
\bar{q}_{1}
\end{array}\right)=\left(\begin{array}{c}
Q_{2}\left(\epsilon_{1}\right) \\
\bar{q}_{2}\left(\epsilon_{1}\right)
\end{array}\right)=\left(\begin{array}{c}
Q \\
\bar{q}
\end{array}\right) \equiv \mathbf{A}^{-1}\left(\begin{array}{c}
A_{0}-e \\
A_{0}-d
\end{array}\right), \forall \epsilon_{1}
$$

Note that quantities at $t=2$ do not fluctuate as functions of the demand shock when there is full confidence in the model. This is a consequence of the lack of persistence $(\rho=0)$ in the particular example (otherwise there would be some dependence on the realization of the demand shock at $t=1)$. Furthermore, note that, even if the monopolist had doubts about the model $\left(\sigma_{R}<0\right)$, as long as the fringe had full confidence $\left(\sigma_{A}=0\right)$, we get the matrix $\mathbf{A}$ in (A.42). Thus, the quantities at $t=2$ for the case of $\left(\sigma_{R}<0, \sigma_{A}=0\right)$ are the same as for the full confidence case of $\sigma_{R}=\sigma_{A}=0$ in (A.43). Consequently, any variation across shocks in the quantities at $t=2$ is coming from the desire of the monopolist to manage the expectations of the fringe (through $\tilde{\xi}_{1}^{V}$ ) and not from any doubts of the monopolist about the model. ${ }^{25}$

Passive stance of the monopolist. Assume that the monopolist were naive and did not realize that the worst-case beliefs of the household were endogenous. This would imply $\tilde{\xi}_{t}^{V}=0$ and therefore $\tilde{\xi}_{t}^{i}$ would be given by (45). So, we would have a naive version of matrix $\mathbf{A}_{\mathbf{t}}$, which is exactly the same as the respective matrix when the fringe has full confidence in the model in (A.42), $\mathbf{A}_{\mathbf{t}}{ }^{\text {naive }}=\mathbf{A}, t=1,2$. The matrix $\mathbf{B}_{\mathbf{t}}$ in (A.16) will remain the same though (and therefore the respective $C_{t}$ in (A.39)). We can follow the same algorithm and use matrix $\mathbf{A}$ to solve for quantities and the worst-case beliefs of the monopolist and the fringe. At each iteration we update the worst-case beliefs and the vector $C_{0}$ (and not $\tilde{\xi}_{1}^{V}\left(\epsilon_{1}\right)$ anymore). Obviously the quantities at $t=2$ are given by (A.43).

Calibration. We use Ljungqvist and Sargent (2004) and Hansen and Sargent (2008) for most of the parameters. The demand parameters are $\left(A_{0}, A_{1}\right)=(100,1)$ and we set $\beta=1$. The reference distribution has mean zero, unitary variance and its support is discretized with eleven points. We set $C_{\epsilon}=0.2 / \sqrt{1-0.8^{2}}=0.33$, which is the unconditional variance of the shock in Ljungqvist and Sargent (2004). The intercepts and slopes of the marginal cost functions are as in Hansen and Sargent (2008), $(d, e)=(20,20)$ and $(g, h)=(1,1)$.

${ }^{25}$ The quantities at $t=1$ would be different from (A.43) in the case of $\sigma_{R}<0, \sigma_{A}=0$ :

$$
\left(\begin{array}{c}
Q_{1} \\
\bar{q}_{1}
\end{array}\right)=\mathbf{A}^{-1}\left(\begin{array}{c}
A_{0}-e+C_{\epsilon} E n_{1}^{*} \epsilon_{1} \\
A_{0}-d
\end{array}\right) .
$$




\section{B Fiscal policy problem}

\section{B.1 Optimality conditions of the fiscal policy problem}

Define for convenience

$$
\Omega(c, h) \equiv U_{c}(c, 1-h) c-U_{l}(c, 1-h) h,
$$

which stands for the equilibrium government surplus in marginal utility units.

The Lagrangian of the fiscal policy problem is

$$
\begin{aligned}
& L=\sum_{t=0}^{\infty} \sum_{g^{t}} \beta^{t} \pi_{t}\left(g^{t}\right)\left\{N_{t}\left(g^{t}\right)\left(U\left(c_{t}\left(g^{t}\right), 1-h_{t}\left(g^{t}\right)\right)+\beta \theta_{R} \sum_{g_{t+1}} \pi_{t+1}\left(g_{t+1} \mid g^{t}\right) n_{t+1}\left(g^{t+1}\right) \ln n_{t+1}\left(g^{t+1}\right)\right)\right. \\
& +\Phi M_{t}^{*}\left(g^{t}\right) \Omega\left(c_{t}\left(g^{t}\right), h_{t}\left(g^{t}\right)\right)-\lambda_{t}\left(g^{t}\right)\left[c_{t}\left(g^{t}\right)+g_{t}-h_{t}\left(g^{t}\right)\right] \\
& -\sum_{g_{t+1}} \beta \pi_{t+1}\left(g_{t+1} \mid g^{t}\right) \mu_{t+1}\left(g^{t+1}\right)\left[M_{t+1}^{*}\left(g^{t+1}\right)-\frac{\exp \left(\sigma_{A} V_{t+1}\left(g^{t+1}\right)\right)}{\sum_{g_{t+1}} \pi_{t+1}\left(g_{t+1} \mid g^{t}\right) \exp \left(\sigma_{A} V_{t+1}\left(g^{t+1}\right)\right)} M_{t}^{*}\left(g^{t}\right)\right] \\
& -\xi_{t}\left(g^{t}\right)\left[V_{t}\left(g^{t}\right)-U\left(c_{t}\left(g^{t}\right), 1-h_{t}\left(g^{t}\right)\right)-\frac{\beta}{\sigma_{A}} \ln \sum_{g_{t+1}} \pi_{t+1}\left(g_{t+1} \mid g^{t}\right) \exp \left(\sigma_{A} V_{t+1}\left(g^{t+1}\right)\right)\right] \\
& -\beta \sum_{g_{t+1}} \pi_{t+1}\left(g_{t+1} \mid g^{t}\right) \rho_{t+1}\left(g^{t+1}\right)\left[N_{t+1}\left(g^{t+1}\right)-n_{t+1}\left(g^{t+1}\right) N_{t}\left(g^{t}\right)\right] \\
& \left.-\nu_{t}\left(g^{t}\right)\left[\sum_{g_{t+1}} \pi_{t+1}\left(g_{t+1} \mid g^{t}\right) n_{t+1}\left(g^{t+1}\right)-1\right]\right\}-\Phi U_{c}\left(c_{0}, 1-h_{0}\right) b_{0},
\end{aligned}
$$

with $\xi_{0}=0, M_{0}=N_{0}=1$ and $g_{0}$ given.

The policymaker's minimization problem with respect to the worst-case beliefs has the same structure as the minimization problem of the household. See Karantounias (2013) for a detailed derivation of the household's worst-case belief distortions in (58). The first-order necessary conditions for an interior solution arising from the maximization problem are the following: 


$$
\begin{array}{cl}
c_{t}: \quad & \left(N_{t}^{*}\left(g^{t}\right)+\xi_{t}\left(g^{t}\right)\right) U_{c}\left(g^{t}\right)+\Phi M_{t}^{*}\left(g^{t}\right) \Omega_{c}\left(g^{t}\right)=\lambda_{t}\left(g^{t}\right), t \geq 1 \\
h_{t}: \quad & \left(N_{t}^{*}\left(g^{t}\right)+\xi_{t}\left(g^{t}\right)\right) U_{l}\left(g^{t}\right)-\Phi M_{t}^{*}\left(g^{t}\right) \Omega_{h}\left(g^{t}\right)=\lambda_{t}\left(g^{t}\right), t \geq 1 \\
M_{t}^{*}: \quad \mu_{t}\left(g^{t}\right)=\Phi \Omega\left(g^{t}\right)+\beta \sum_{g_{t+1}} \pi_{t+1}\left(g_{t+1} \mid g^{t}\right) m_{t+1}^{*}\left(g^{t+1}\right) \mu_{t+1}\left(g^{t+1}\right), t \geq 1 \\
V_{t}: \quad \xi_{t}\left(g^{t}\right)=\sigma_{A} m_{t}^{*}\left(g^{t}\right) M_{t-1}^{*}\left(g^{t-1}\right)\left[\mu_{t}\left(g^{t}\right)-\sum_{g_{t}} \pi_{t}\left(g_{t} \mid g^{t-1}\right) m_{t}^{*}\left(g^{t}\right) \mu_{t}\left(g^{t}\right)\right] \\
\quad+m_{t}^{*}\left(g^{t}\right) \xi_{t-1}\left(g^{t-1}\right), t \geq 1 \\
\quad\left(N_{0}+\xi_{0}\right) U_{c}\left(g_{0}\right)+\Phi M_{0} \Omega_{c}\left(g_{0}\right)=\lambda_{0}\left(g_{0}\right)+\Phi U_{c c}\left(g_{0}\right) b_{0} \\
c_{0}: \quad\left(N_{0}+\xi_{0}\right) U_{l}\left(g_{0}\right)+\Phi M_{0} \Omega_{h}\left(g_{0}\right)=-\lambda_{0}\left(g_{0}\right)-\Phi U_{c l}\left(g_{0}\right) b_{0} .
\end{array}
$$

$\Omega_{i}, i=c, h$ denotes the respective partial derivative of the government surplus. In (B.4) and (B.5), we used expression (58) for the optimal conditional likelihood ratio $m_{t+1}^{*}$ to save notation. Optimality conditions (70-71), (B.2-B.7), together with constraints (64-69) determine the Ramsey plan.

By letting $\tilde{\xi}_{t} \equiv \xi_{t} / N_{t}^{*}$ denote the normalized multiplier and recalling the law of motion (72), we can rewrite (B.5) as in the law of motion (76) in the text. Furthermore, solving (B.4) forward and remembering that $\Omega\left(g^{t}\right)$ stands for the government surplus in marginal utility terms leads to

$$
\mu_{t}\left(g^{t}\right)=\Phi U_{c}\left(g^{t}\right) \sum_{i=0}^{\infty} \sum_{g^{t+i} \mid g^{t}} q_{t+i}^{t}\left(g^{t+i}\right)\left[\tau_{t+i}\left(g^{t+i}\right) h_{t+i}\left(g^{t+i}\right)-g_{t+i}\right]
$$

where

$$
q_{t+i}^{t}\left(g^{t+i}\right) \equiv \frac{q_{t+i}\left(g^{t+i}\right)}{q_{t}\left(g^{t}\right)}=\beta^{i} \pi_{t+i}\left(g^{t+i} \mid g^{t}\right) \prod_{j=1}^{i} m_{t+j}^{*}\left(g^{t+j}\right) \frac{U_{c}\left(g^{t+i}\right)}{U_{c}\left(g^{t}\right)}
$$

the equilibrium price of an Arrow-Debreu security at $g^{t}$. Thus, using the intertemporal budget constraint at time $t$, allows us to rewrite $\mu_{t}$ in terms of debt as $\mu_{t}\left(g^{t}\right)=\Phi U_{c}\left(g^{t}\right) b_{t}\left(g^{t}\right)$. 


\section{B.2 Proof of proposition 4}

Proof. Combine (B.2) and (B.3) in order to eliminate the multiplier $\lambda_{t}$ to get

$$
\frac{U_{l}}{U_{c}} \cdot \frac{N_{t}^{*}+\xi_{t}-\Phi M_{t}^{*} \frac{\Omega_{h t}}{U_{l t}}}{N_{t}^{*}+\xi_{t}+\Phi M_{t}^{*} \frac{\Omega_{c t}}{U_{c t}}}=1
$$

Recall that $\tilde{\xi}_{t} \equiv \xi_{t} / N_{t}^{*}$. Calculate the derivatives $\Omega_{i}$ to get $\Omega_{c} / U_{c}=1-\epsilon_{c c}-\epsilon_{c h}$ and $\Omega_{h} / U_{l}=-1-\epsilon_{h h}-\epsilon_{h c}$. We can rewrite (B.8) as

$$
\left(1-\tau_{t}\right) \cdot \frac{1+\tilde{\xi}_{t}+\Phi \Lambda_{t}\left[1+\epsilon_{h h, t}+\epsilon_{h c, t}\right]}{1+\tilde{\xi}_{t}+\Phi \Lambda_{t}\left[1-\epsilon_{c c, t}-\epsilon_{c h, t}\right]}=1
$$

Solve in terms of $\tau_{t}$ to get (73) in the text. Furthermore, by using the expression for $\Omega_{h} / U_{l}$, the first-order condition for labor (B.3) can be rewritten as

$$
1+\tilde{\xi}_{t}+\Phi \Lambda_{t}\left[1+\epsilon_{h h, t}+\epsilon_{h c, t}\right]=\frac{\lambda_{t}}{U_{l t} N_{t}^{*}}>0
$$

since $\lambda_{t}>0$. Thus, the denominator in (73) is positive, despite the fact that $\tilde{\xi}_{t}$ can take negative values. When $U_{c l} \geq 0$, the numerator in the optimal tax formula is also positive, so in that case we get $\tau_{t} \geq 0$. The expressions in (74) and (75) are an application of the formula in (73). 


\section{References}

Ajello, Andrea, Thomas Laubach, David López-Salido, and Taisuke Nakata. 2019. Financial Stability and Optimal Interest Rate Policy. International Journal of Central Banking 15 (1):279326.

Bachmann, Ruediger, Kai Carstensen, Stefan Lautenbacher, and Martin Schneider. 2020. Uncertainty is more than risk - survey evidence on Knightian and Bayesian firms. Mimeo, Stanford University.

Barlevy, Gadi. 2009. Policymaking under Uncertainty: Gradualism and Robustness. Economic Pespectives Q II:38-55. Federal Reserve Bank of Chicago.

—. 2011. Robustness and Macroeconomic Policy. Annual Review of Economics 3:1-24.

Benigno, Pierpaolo and Salvatore Nisticò. 2012. International portfolio allocation under model uncertainty. American Economic Journal: Macroeconomics 4 (1):144-89.

Benigno, Pierpaolo and Luigi Paciello. 2014. Monetary policy, doubts and asset prices. Journal of Monetary Economics 64:85-98.

Bhandari, Anmol, Jaroslav Borovička, and Paul Ho. 2019. Survey data and subjective beliefs in business cycle models. Mimeo, New York University.

Bidder, Rhys M. and Matthew E. Smith. 2012. Robust animal spirits. Journal of Monetary Economics 59 (8):738-750.

Christensen, Timothy M. 2019. Dynamic Models with Robust Decision Makers: Identification and Estimation. Mimeo, New York University.

Cogley, Timothy, Riccardo Colacito, Lars Peter Hansen, and Thomas J. Sargent. 2008. Robustness and U.S. Monetary Policy Experimentation. Journal of Money, Credit and Banking 40 (8):1599-1623.

Collard, Fabrice, Sujoy Mukerji, Kevin Sheppard, and Jean-Marc Tallon. 2018. Ambiguity and the historical equity premium. Quantitative Economics 9:945-993.

Croce, Mariano M., Thien T. Nguyen, and Lukas Schmid. 2012. The market price of fiscal uncertainty. Journal of Monetary Economics 59 (5):401-416.

Dennis, Richard. 2008. Robust control with commitment: A modification to Hansen-Sargent. Journal of Economic Dynamics and Control 32 (7):2061-2084. 
Dow, James and Sérgio Ribeiro da Costa Werlang. 1992. Uncertainty Aversion, Risk Aversion, and the Optimal Choice of Portfolio. Econometrica 60 (1):197-204.

Ferrière, Axelle and Anastasios G. Karantounias. 2019. Fiscal austerity in ambiguous times. American Economic Journal: Macroeconomics 11 (1):89-131.

Giannoni, Marc P. 2002. Does Model Uncertainty Justify Caution? Robust Optimal Monetary Policy in a Forward- Looking Model. Macroeconomic Dynamics 6 (1):111-144.

2007. Robust Optimal Monetary Policy in a Forward-Looking Model with Parameter and Shock Uncertainty. Journal of Applied Econometrics 22 (1):179-213.

Hansen, Lars Peter and Thomas J. Sargent. 2001. Robust Control and Model Uncertainty. American Economic Review 91 (2):60-66.

- 2005. Robust Estimation and Control under Commitment. Journal of Economic Theory $124(2): 258-301$.

—. 2007. Recursive Robust estimation and Control without Commitment. Journal of Economic Theory 136 (1):1-27.

- 2008. Robustness. Princeton University Press.

- 2010. Fragile beliefs and the price of uncertainty. Quantitative Economics 1:129-162.

—. 2012. Three types of ambiguity. Journal of Monetary Economics 59 (5):422-445.

- 2020. Structured ambiguity and model misspecification. Mimeo, University of Chicago and New York University.

Hansen, Lars Peter, Thomas J. Sargent, Gauhar Turmuhambetova, and Noah Williams. 2006. Robust Control and Model Misspecification. Journal of Economic Theory 128:45-90.

Hansen, Lars Peter, John Heaton, Junghoon Lee, and Nikolai Roussanov. 2007. Intertemporal Substitution and Risk Aversion. Handbook of Econometrics 6A.

Holmes, Mark H. 1996. Introduction to Perturbation Methods. Springer-Verlag.

Ilut, Cosmin L. and Martin Schneider. 2014. Ambiguous business cycles. American Economic Review 104 (8):2368-99.

Ju, Nengjiu and Jianjun Miao. 2012. Ambiguity, learning and asset returns. Econometrica $80(2): 559-591$.

Judd, Kenneth L. 1998. Numerical Methods in Economics. MIT Press. 
Karantounias, Anastasios G. 2013. Managing pessimistic expectations and fiscal policy. Theoretical Economics 8 (1):193-231.

Kogan, Leonid and Raman Uppal. 2002. Risk Aversion and Optimal Portfolio Policies in Partial and General Equilibrium Economies. Mimeo, Sloan School of Management and London Business School.

Kwon, Hyosung and Jianjun Miao. 2017. Three types of robust Ramsey problems in a linearquadratic framework. Journal of Economic Dynamics and Control 76:211-231.

Kydland, Finn E. and Edward C. Prescott. 1980. Dynamic optimal taxation, rational expectations and optimal control. Journal of Economic Dynamics and Control 2:79-91.

Ljungqvist, Lars and Thomas J. Sargent. 2004. Recursive Macroeconomic Theory. MIT Press, 2nd edition.

Lucas, Robert Jr. and Nancy L. Stokey. 1983. Optimal fiscal and monetary policy in an economy without capital. Journal of Monetary Economics 12 (1):55-93.

Luo, Yulei, Jun Nie, and Eric R. Young. 2014. Model uncertainty and intertemporal tax smoothing. Journal of Economic Dynamics and Control 45:289-314.

Marcet, Albert and Ramon Marimon. 2019. eEcursive Contracts. Econometrica 87 (5):15891631.

Michelacci, Claudio and Luigi Paciello. 2019. Ambiguous policy announcements. Review of Economic Studies Forthcoming.

— 2020. Aggregate risk or aggregate uncertainty? Evidence from UK households. Mimeo, Einaudi Insitute for Economics and Finance.

Molavi, Pooya. 2019. Macroeconomics with Learning and Misspecification: A General Theory and Applications. Mimeo, Massachusetts Insitute of Technology.

Orlik, Anna and Ignacio Presno. 2018. On credible monetary policies under model uncertainty. Mimeo, Board of Governors of the Federal Reserve System.

Orphanides, Athanasios and John C. Williams. 2007. Robust monetary policy with imperfect knowledge. Journal of Monetary Economics 54:1406-1435.

Pouzo, Demian and Ignacio Presno. 2016. Sovereign Default Risk and Uncertainty Premia. American Economic Journal: Macroeconomics 8 (3):230-66. 
Strzalecki, Tomasz. 2011. Axiomatic Foundations of Multiplier Preferences. Econometrica $79(1): 47-73$.

Tetlow, Robert J. 2015. Real-Time Model Uncertainty in the United States: 'Robust' Policies Put to the Test. International Journal of Central Banking 11 (1):113-155.

Tetlow, Robert J. and Peter von zur Muehlen. 2001. Robust Monetary Policy with Misspecified Models: Does Model Uncertainty always Call for Attenuated Policy? Journal of Economic Dynamics and Control 25:911-949.

Woodford, Michael. 2010. Robustly Optimal Monetary Policy with Near-Rational Expectations. American Economic Review 100 (1):274-303. 


\title{
Doubts about the model and optimal policy
}

\author{
Anastasios G. Karantounias ${ }^{1}$
}

July 23,2020

\section{Online Appendix \\ (not for publication)}

Keywords: Model uncertainty, ambiguity aversion, multiplier preferences, misspecification, robustness, martingale, monopolist, competitive fringe, demand uncertainty, Ramsey taxation.

JEL classification: D80; E62; H21; H63.

\footnotetext{
${ }^{1}$ Research Department, Federal Reserve Bank of Atlanta, 1000 Peachtree St NE, Atlanta, GA 30309, USA. Email: anastasios.karantounias@atl.frb.org. All errors are my own. The views expressed herein are those of the author and not necessarily those of the Federal Reserve Bank of Atlanta or the Federal Reserve System.
} 


\section{Small-doubts approximation}

\section{Contents}

C.1 Logic of expansion and some caveats . . . . . . . . . . . . . iii

C.2 Proof of proposition $5 \ldots \ldots \ldots \ldots$. . . . . . . . . . iv

C.3 Useful definitions and facts . . . . . . . . . . . . . . . . . . . . vii

C.3.1 Second-order conditions . . . . . . . . . . . . . . . . vii

C.3.2 Optimal wedge . . . . . . . . . . . . . . . ix

C.3.3 History independence of the full-confidence allocation . . . . . . . . $\quad \mathrm{x}$

C.3.4 Some simplifications . . . . . . . . . . . . . . . $\quad \mathrm{x}$

C.4 Consumption and labor . . . . . . . . . . . . . . . . . . xi

C.5 Cost of distortionary taxation . . . . . . . . . . . . . . . . xiii

C.6 Tax rate . . . . . . . . . . . . . . . . . . . . xiv

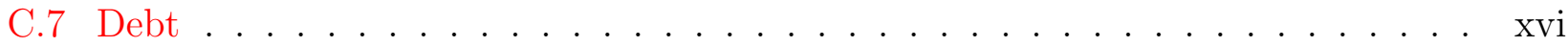

C.8 Formulas for Markov shocks . . . . . . . . . . . . . . . . . . . . xix

C.9 Quasi-linear utility ....................... . xxi

C.9.1 No doubts about the model . . . . . . . . . . . . . . xxi

C.9.2 Proof of proposition $6 \ldots \ldots \ldots$. . . . . . . . . . . . . . . . . . . . . . . . .

C.9.3 Proof of proposition $7 \ldots \ldots \ldots \ldots$. . . . . . . . . . . . . . . . 


\section{C.1 Logic of expansion and some caveats}

We express every endogenous variable (either random or non-random as the multiplier $\Phi$ ) as function of the parameter vector $\sigma=\left(\sigma_{R}, \sigma_{A}\right)$. The first-order expansion for a generic variable $x_{t}$ around $\left(\sigma_{R}, \sigma_{A}\right)=(0,0)$ takes the form

$$
x_{t}\left(g^{t}, \sigma_{R}, \sigma_{A}\right) \simeq x_{t}\left(g^{t}, 0,0\right)+\sigma_{R} x_{\sigma_{R}}\left(g^{t}, 0,0\right)+\sigma_{A} x_{\sigma_{A}}\left(g^{t}, 0,0\right)
$$

where $x_{t}\left(g^{t}, 0,0\right)$ refers to the respective variable in the Lucas and Stokey (1983) economy. For convenience, we use the notation $x_{t}(\sigma) \equiv x_{t}\left(g^{t}, \sigma\right)$ and $x_{t}^{i}(\sigma) \equiv x_{\sigma_{i}}\left(g^{t}, \sigma\right), i=R, A$, with $x_{t}(0)$ and $x_{t}^{i}(0), i=R, A$ the respective evaluation at $\sigma=(0,0)$.

The Lucas and Stokey (1983) plan is easy to calculate because it is essentially static. This is due to its history-independence property for variables like consumption, labor and the tax rate, $x_{t}\left(g^{t}, 0,0\right)=x\left(g_{t}, 0,0\right) .^{2}$ The expansion is focused on the calculation of the partial derivatives $x_{t}^{i}(0), i=R, A$, which are random variables in most cases. Substantial simplification comes from the fact that, without doubts about the model, the conditional and unconditional likelihood ratios become unity, $m_{t}^{*}(0)=n_{t}^{*}(0)=M_{t}^{*}(0)=N_{t}^{*}(0)=\Lambda_{t}(0)=1$. Futhermore, there is no room for price manipulation through continuation utilities, so $\tilde{\xi}_{t}(0)=0$, and the government's and household's utility coincide, $W_{t}(0)=V_{t}(0)$, since both the government and the household share the same reference model.

Caveats and caution. We want to draw here some caution on the results of the smalldoubts expansion. The optimal plan with model uncertainty is driven by the state variables $\left(\Lambda_{t}, \tilde{\xi}_{t}\right)$, which summarize the history $g^{t}$. In the full-confidence economy these state variables are constant (reflecting the history-independence of Lucas and Stokey (1983)), so this type of perturbation is singular in the terminology of Holmes (1996). Moreover, the state variables, which are martingales, become random walks in the expansion, as seen in proposition 5. So, in a sense, we approximate a non-stationary economy by using information from the stationary counterpart at $\sigma=(0,0)$. We are not worried so much about the persistence indicated by the random walk result. This was expected, given the martingale nature of the state variables. ${ }^{3}$ More worrisome that the fact that some variables, like the tax rate, will surpass $100 \%$ after a sufficiently long time.

How should we use the expansion? For both of the reasons stated in the previous paragraph, we consider the heuristic expansions as valid only for the short-run, that is, for a limited number of periods, starting from $t=0$ (and not from some long-run 'steady state'). This is

\footnotetext{
${ }^{2}$ The history-independence property extends also to debt if we assume Markovian shocks.

${ }^{3}$ Note that persistence is also very high in problems where a global solution method is used. See for example Ferrière and Karantounias (2019).
} 
both to avoid the explosiveness in the long-run, and also to limit the error accumulated from the expansion. To elaborate on the second point, proposition 5 shows that the increment to the multiplier $\tilde{\xi}_{t}$ is determined by the Lucas and Stokey debt position. But if we had a realization of good shocks over time, which would lead to an increasing amount of debt over time, the fullconfidence debt position would not be a good approximation anymore. Initializing the economy from $t=0$ and constraining the number of periods deals with these issues.

\section{C.2 Proof of proposition 5}

Part 1. Consider first the belief ratio as function of the vector $\sigma \equiv\left(\sigma_{R}, \sigma_{A}\right), \Lambda_{t}(\sigma) \equiv \frac{M_{t}^{*}(\sigma)}{N_{t}^{*}(\sigma)}$. Differentiate with respect to $\sigma_{i}, i=R, A$ to get

$$
\Lambda_{t}^{i}(\sigma)=\frac{M_{t}^{* i}(\sigma) N_{t}^{*}(\sigma)-M_{t}^{*}(\sigma) N_{t}^{* i}(\sigma)}{\left(N_{t}^{*}(\sigma)\right)^{2}}, i=R, A
$$

Evaluate at $\sigma=(0,0)$ to get

$$
\Lambda_{t}^{i}(0)=M_{t}^{* i}(0)-N_{t}^{* i}(0), i=R, A
$$

Consider the martingale distortions $N_{t}^{*}$ and $M_{t}^{*}$, with laws of motion $N_{t}^{*}(\sigma)=n_{t}^{*}(\sigma) N_{t-1}^{*}(\sigma)$ and $M_{t}^{*}(\sigma)=m_{t}^{*}(\sigma) M_{t-1}^{*}(\sigma)$, and initial values $N_{0}=M_{0} \equiv 1$. Differentiate the law of motion of $N_{t}^{*}$ with respect to $\sigma_{i}, i=R, A$

$$
N_{t}^{* i}(\sigma)=n_{t}^{* i}(\sigma) N_{t}^{*}(\sigma)+n_{t}^{*}(\sigma) N_{t-1}^{* i}(\sigma), N_{0}^{* i}(0) \equiv 0, i=R, A
$$

Thus, at $\sigma=(0,0)$ we get

$$
N_{t}^{* i}(0)=n_{t}^{* i}(0)+N_{t-1}^{* i}(0), N_{0}^{* i}(0) \equiv 0, i=R, A
$$

Repeating exactly the same steps for the martingale $M_{t}^{*}$ delivers

$$
M_{t}^{* i}(0)=m_{t}^{* i}(0)+M_{t-1}^{* i}(0), M_{0}^{* i}(0) \equiv 0, i=R, A
$$

Consider now the conditional likelihood ratios $\left(n_{t}^{*}, m_{t}^{*}\right)$. We have

$$
n_{t}^{*}(\sigma)=\frac{\exp \left(\sigma_{R} W_{t}(\sigma)\right)}{E_{t-1} \exp \left(\sigma_{R} W_{t}(\sigma)\right)} \quad \text { and } \quad m_{t}^{*}(\sigma)=\frac{\exp \left(\sigma_{A} V_{t}(\sigma)\right)}{E_{t-1} \exp \left(\sigma_{A} V_{t}(\sigma)\right)}
$$


The increments $n_{t}^{* i}(\sigma), m_{t}^{* i}(\sigma), i=R, A$ to the martingale derivatives are

$$
\begin{aligned}
n_{t}^{* R}(\sigma) & =n_{t}^{*}(\sigma)\left[W_{t}(\sigma)+\sigma_{R} W_{t}^{R}(\sigma)-E_{t-1} n_{t}^{*}(\sigma)\left[W_{t}(\sigma)+\sigma_{R} W_{t}^{R}(\sigma)\right]\right] \\
n_{t}^{* A}(\sigma) & =\sigma_{R} n_{t}^{*}(\sigma)\left[W_{t}^{A}(\sigma)-E_{t-1} n_{t}^{*}(\sigma) W_{t}^{A}(\sigma)\right] \\
m_{t}^{* R}(\sigma) & =\sigma_{A} m_{t}^{*}(\sigma)\left[V_{t}^{R}(\sigma)-E_{t-1} m_{t}^{*}(\sigma) V_{t}^{R}(\sigma)\right] \\
m_{t}^{* A}(\sigma) & =m_{t}^{*}(\sigma)\left[V_{t}(\sigma)+\sigma_{A} V_{t}^{A}(\sigma)-E_{t-1} m_{t}^{*}(\sigma)\left[V_{t}(\sigma)+\sigma_{A} V_{t}^{A}(\sigma)\right]\right]
\end{aligned}
$$

Evaluating now at $\sigma=(0,0)$ delivers

$$
\begin{aligned}
& n_{t}^{* R}(0)=m_{t}^{* A}(0)=V_{t}(0)-E_{t-1} V_{t}(0) \\
& n_{t}^{* A}(0)=m_{t}^{* R}(0)=0
\end{aligned}
$$

Therefore, using (C.5) and (C.6), the unconditional martingale derivatives in (C.3) and (C.4) become

$$
\begin{aligned}
& M_{t}^{* A}(0)=N_{t}^{* R}(0)=\sum_{j=1}^{t}\left(V_{j}(0)-E_{j-1} V_{j}(0)\right) \\
& N_{t}^{* A}(0)=M_{t}^{* R}(0)=0
\end{aligned}
$$

Thus, (C.2), (C.7) and (C.8) imply that

$$
\begin{aligned}
\Lambda_{t}^{A}(0) & =M_{t}^{* A}(0) \\
\Lambda_{t}^{R}(0) & =-N_{t}^{* R}(0)=-M_{t}^{* A}(0) .
\end{aligned}
$$

Use now (C.1) to get

$$
\Lambda_{t}=1+\sigma_{A} \Lambda_{t}^{A}(0)+\sigma_{R} \Lambda_{t}^{R}(0)=1+\left(\sigma_{A}-\sigma_{R}\right) M_{t}^{* A}(0)
$$

Evaluate (C.11) at $t-1$, take differences and use (C.5) for the martingale derivative increment to get $(80)$ in the text.

Part 2. Define for convenience the innovation in $\mu_{t}$ (under the household's worst-case measure), 


$$
\eta_{t} \equiv \mu_{t}-E_{t-1} m_{t}^{*} \mu_{t}
$$

and rewrite the law of motion of the multiplier $\tilde{\xi}_{t}$ in (76) as

$$
\tilde{\xi}_{t}(\sigma)=\sigma_{A} \eta_{t}(\sigma) \Lambda_{t}(\sigma)+\frac{m_{t}^{*}(\sigma)}{n_{t}^{*}(\sigma)} \tilde{\xi}_{t-1}(\sigma), \tilde{\xi}_{0} \equiv 0
$$

Differentiate with respect to $\sigma$ to get

$$
\begin{aligned}
\tilde{\xi}_{t}^{R}(\sigma)= & \sigma_{A}\left[\eta_{t}^{R}(\sigma) \Lambda_{t}(\sigma)+\eta_{t}(\sigma) \Lambda_{t}^{R}(\sigma)\right]+\frac{m_{t}^{* R}(\sigma) n_{t}^{*}-m_{t}^{*}(\sigma) n_{t}^{* R}(\sigma)}{\left(n_{t}^{*}(\sigma)\right)^{2}} \tilde{\xi}_{t-1}(\sigma) \\
& +\frac{m_{t}^{*}(\sigma)}{n_{t}^{*}(\sigma)} \tilde{\xi}_{t-1}^{R}(\sigma) \\
\tilde{\xi}_{t}^{A}(\sigma)= & \eta_{t}(\sigma) \Lambda_{t}(\sigma)+\sigma_{A}\left[\eta_{t}^{A}(\sigma) \Lambda_{t}(\sigma)+\eta_{t}(\sigma) \Lambda_{t}^{A}(\sigma)\right]+\frac{m_{t}^{* A}(\sigma) n_{t}^{*}-m_{t}^{*}(\sigma) n_{t}^{* A}(\sigma)}{\left(n_{t}^{*}(\sigma)\right)^{2}} \tilde{\xi}_{t-1}(\sigma) \\
& +\frac{m_{t}^{*}(\sigma)}{n_{t}^{*}(\sigma)} \tilde{\xi}_{t-1}^{A}(\sigma),
\end{aligned}
$$

with $\tilde{\xi}_{0}^{i}(0) \equiv 0, i=R, A$.

Evaluate (C.13) and (C.14) at $\sigma=(0,0)$ (recalling the unitary likelihood ratios and $\left.\tilde{\xi}_{t}(0)=0\right)$ to get

$$
\begin{aligned}
& \tilde{\xi}_{t}^{R}(0)=0 \\
& \tilde{\xi}_{t}^{A}(0)=\eta_{t}(0)+\tilde{\xi}_{t-1}^{A}(0) \Rightarrow \tilde{\xi}_{t}^{A}(0)=\sum_{i=1}^{t} \eta_{i}(0) .
\end{aligned}
$$

Recall definition (C.12) and the fact that $\mu_{t}=\Phi U_{c t} b_{t}$ (from (77)) to get

$$
\eta_{t}(0)=\mu_{t}(0)-E_{t-1} \mu_{t}(0)=\Phi(0)\left[U_{c t}(0) b_{t}(0)-E_{t-1} U_{c t}(0) b_{t}(0)\right],
$$

where $\Phi(0)$ the respective cost of distortionary taxation in the economy without model uncertainty. Use now (C.1) to get $\tilde{\xi}_{t}=\sigma_{A} \tilde{\xi}_{t}^{A}(0)$, which implies the approximate law of motion in the text (81). 


\section{C.3 Useful definitions and facts}

\section{C.3.1 Second-order conditions}

For the rest, bear in mind the following definitions:

$$
\begin{aligned}
& K_{t}(0) \equiv(1+2 \Phi(0)) \Delta\left(c_{t}(0)\right)+\Phi(0)\left[\Delta^{\prime}\left(c_{t}(0)\right) c_{t}(0)+\Gamma^{\prime}\left(c_{t}(0)\right) g_{t}\right], t \geq 1 \\
& K_{0}(0) \equiv(1+2 \Phi(0)) \Delta\left(c_{0}(0)\right)+\Phi(0)\left[\Delta^{\prime}\left(c_{0}(0)\right) c_{0}(0)+\Gamma^{\prime}\left(c_{0}(0)\right) g_{0}-Z^{\prime}\left(c_{0}(0)\right) b_{0}\right]
\end{aligned}
$$

where

$$
\begin{aligned}
\Delta\left(c_{t}\right) & \equiv U_{c c t}-2 U_{c l t}+U_{l l t}<0 \\
\Gamma\left(c_{t}\right) & \equiv U_{l l t}-U_{c l t} \\
Z\left(c_{t}\right) & \equiv U_{c c t}-U_{c l t}
\end{aligned}
$$

Note that we have already substituted for labor from the resource constraint, so all expressions above should be understood as functions of consumption only and in particular of the full confidence consumption allocation, $c_{t}(0)$. The term $\Delta\left(c_{t}\right)$ is negative due to the concavity of the period utility function. $\Delta^{\prime}()$ stands for the derivative of the particular expression with respect to consumption. $\Delta_{t}^{\prime}\left(c_{t}(0)\right)$ denotes the evaluation of the derivative at the consumption of the no doubts economy. The same notational interpretations hold for $\Gamma^{\prime}, Z^{\prime}$.

Assumption 1. $K_{t}(0)<0, \forall t \geq 0$.

We will work under assumption 1 for the rest of the expansion. $K_{t}(0)$ is an expression that is directly related to the second derivative of the Lagrangian of the problem without doubts about the model and is intimately connected to the sufficient second-order conditions of the Lucas and Stokey problem.

Lemma 1. If assumption 1 holds, then the second-order sufficient conditions of the optimal fiscal policy problem without fear of misspecification are satisfied.

Proof. Let $l(c, \Phi) \equiv U(c, 1-c-g)+\Phi\left[\left(U_{c}(c, 1-c-g)-U_{l}(c, 1-c-g)\right) c-U_{l}(c, 1-c-g) g\right]$ denote the period return in the lagrangian for the Lucas and Stokey economy for $t \geq 1$ and let $l^{0}\left(c, \Phi, b_{0}\right) \equiv U\left(c, 1-c-g_{0}\right)+\Phi\left[\left(U_{c}\left(c, 1-c-g_{0}\right)-U_{l}\left(c, 1-c-g_{0}\right)\right) c-U_{l}\left(c, 1-c-g_{0}\right) g_{0}-\right.$ $\left.U_{c}\left(c, 1-c-g_{0}\right) b_{0}\right]$ denote the respective lagrangian for $t=0$. It is easy to see that $l_{c c}=K$ and $l_{c c}^{0}=K_{0}$ where $K$ and $K_{0}$ the expressions in (C.18) and (C.19). We drop the 0 notation since it is clear that we work with the allocation of the no-robustness case. The second-order sufficient conditions require the Hessian of the Lagrangian with respect to $c_{t}\left(g^{t}\right)$ to be negative definite on the tangent plane of the constraint space defined by $A \equiv\left\{x: \sum_{t=0}^{\infty} \sum_{g^{t}} \frac{\partial F(\{c\})}{\partial c_{t}\left(g^{t}\right)} x_{t}\left(g^{t}\right)=0\right\}$, 
where $F(\{c\}) \equiv \sum_{t=0}^{\infty} \sum_{g^{t}} \pi_{t}\left(g^{t}\right)\left[\left(U_{c}\left(g^{t}\right)-U_{l}\left(g^{t}\right)\right) c_{t}\left(g^{t}\right)-U_{l}\left(g^{t}\right) g_{t}\right]-U_{c_{0}} b_{0}$, i.e. the implementabiity constraint (in terms of $c_{t}$ ). All expressions are calculated at $\{c\}$ that is regular and satisfies the first-order conditions. The time separability of the utility function with full confidence in $\pi$ makes the Hessian diagonal, so the second order conditions take the form $\sum_{t=0}^{\infty} \beta^{t} \sum_{g^{t}} K_{t}\left(g^{t}\right) x_{t}^{2}\left(g^{t}\right)<0$ for all $x \neq 0, x \in A$. It is apparent that they are satisfied if $K_{t}<0, \forall t \geq 0$.

Examples with a power function in $h$ or $l$. Consider $K$ in (C.18) which takes a simple form for utility functions with $U_{c l}=0$. In particular,

$$
K=T^{c}-T^{l},
$$

where we have collected the terms involving derivatives of the utility function with respect to consumption and leisure as follows

$$
\begin{aligned}
T^{c} & \equiv(1+2 \Phi) U_{c c}+\Phi U_{c c c} c \\
T^{l} & \equiv-(1+2 \Phi) U_{l l}+\Phi U_{l l l} h .
\end{aligned}
$$

We show now that assumption 1 holds for the utility functions used in proposition 4 ,

$$
\begin{aligned}
U(c, 1-h) & =\frac{c^{1-\gamma}-1}{1-\gamma}-a_{h} \frac{h^{1+\phi_{h}}}{1+\phi_{h}} \\
U(c, l) & =\frac{c^{1-\gamma}-1}{1-\gamma}+a_{l} \frac{l^{1-\psi}-1}{1-\psi} .
\end{aligned}
$$

For the utility function in (C.23) we get

$$
\begin{aligned}
& T^{c}=-\gamma c^{-\gamma-1}(1+\Phi(1-\gamma)) \\
& T^{l}=a_{h} \phi_{h} h^{\phi_{h}-1}\left(1+\Phi\left(1+\phi_{h}\right)\right)>0 .
\end{aligned}
$$

For the utility function in (C.24) $T^{c}$ is the same and

$$
T^{l}=a_{l} \psi l^{-\psi-1}\left[1+\Phi\left(2+(\psi+1) \frac{h}{l}\right)\right]>0 .
$$

Note that by evaluating the first-order condition (B.2) for $\sigma=(0,0)$ we get $c^{-\gamma}(1+\Phi(1-\gamma))=$ $\lambda>0$ and therefore $1+\Phi(1-\gamma)>0$ for the multiplier $\Phi$ that corresponds to the full-confidence economy. Thus, we get $T^{c}<0$ from (C.25), so $K=T^{c}-T^{l}<0$ for both utility functions. Going to the initial period $K_{0}$ in (C.19), $K_{0}=T_{0}^{c}-T_{0}^{l}$, with 


$$
T_{0}^{c}=(1+2 \Phi) U_{c c}+\Phi U_{c c c}\left(c_{0}-b_{0}\right)
$$

and $T_{0}^{l}=T^{l}$. So for the utility functions in (C.23) and (C.24) we have

$$
T_{0}^{c}=-\gamma c_{0}^{-\gamma-2}\left[(1+\Phi(1-\gamma)) c_{0}+\Phi(\gamma+1) b_{0}\right]
$$

Note that when $b_{0} \geq 0$ we have $T_{0}^{c}<0$ and therefore $K_{0}<0$. We are assuming that even in the case of initial assets $b_{0}<0$, their size is not large enough to violate the $K_{0}<0$ condition.

\section{C.3.2 Optimal wedge}

Consider the first-order conditions with respect to $(c, h)$ in (B.2) and (B.3). The partial derivatives of $\Omega$ are

$$
\begin{aligned}
& \Omega_{c}=U_{c}+U_{c c} c-U_{c l} h \\
& \Omega_{h}=-U_{l}+U_{l l} h-U_{c l} c
\end{aligned}
$$

As in the proof of proposition 4, we can eliminate the multiplier $\lambda_{t}$ and combine the two conditions as

$$
U_{l}-U_{c}=\frac{\Phi \Lambda_{t}}{1+\tilde{\xi}_{t}+\Phi \Lambda_{t}}\left[U_{c c} c_{t}-U_{c l}\left(c_{t}+h_{t}\right)+U_{l l} h_{t}\right], t \geq 1 .
$$

Equation (C.31) determines the optimal wedge $\left(U_{l}-U_{c}\right)$ and is just another way of expressing the optimal tax rate $\tau_{t}$. Using the initial period conditions (B.6) and (B.7) and following similar steps delivers the optimal wedge at $t=0$

$$
U_{l 0}-U_{c 0}=\frac{\Phi}{1+\Phi}\left[U_{c c 0}\left(c_{0}-b_{0}\right)-U_{c l 0}\left(c_{0}+h_{0}-b_{0}\right)+U_{l l 0} h_{0}\right] .
$$

We can use the resource constraint (49) to eliminate labor $h_{t}$ and rewrite the optimal wedges as 


$$
\begin{aligned}
U_{l}-U_{c} & =\frac{\Phi \Lambda_{t}}{1+\tilde{\xi}_{t}+\Phi \Lambda_{t}}\left[\Delta\left(c_{t}\right) c_{t}+\Gamma\left(c_{t}\right) g_{t}\right], t \geq 1 . \\
U_{l 0}-U_{c 0} & =\frac{\Phi}{1+\Phi}\left[\Delta\left(c_{0}\right) c_{0}+\Gamma\left(c_{0}\right) g_{0}-Z\left(c_{0}\right) b_{0}\right],
\end{aligned}
$$

where $\Delta, \Gamma, Z$ defined in (C.20), (C.21) and (C.22) respectively. Note that (C.33) and (C.34) are function of consumption only.

\section{C.3.3 History independence of the full-confidence allocation}

The history independence of the Lucas and Stokey (1983) allocation is a well-known result, which we include here for completeness. Consider the optimal wedge (C.31) together with the resource constraint (49) and assume that we are at the full confidence case of $\sigma=(0,0)$. Then, $\Lambda_{t}=1, \tilde{\xi}_{t}=0$, and we can solve from these two equations for consumption and labor as functions only of the current shock and the constant value of the multiplier $\Phi, c_{t}=c\left(g_{t} ; \Phi\right)$, $h_{t}=h\left(g_{t} ; \Phi\right), t \geq 1$. Similarly, we can use (C.32) to solve for the initial consumption and labor as $c_{0}=c\left(g_{0}, b_{0} ; \Phi\right)$ and $h_{0}=h\left(g_{0}, b_{0} ; \Phi\right)$. Thus, consumption and labor (and therefore the tax rate) depend only on the current shock $g_{t}$. The value of the multiplier $\Phi$ has to be adjusted so that the intertemporal budget constraint of the government holds. ${ }^{4}$

\section{C.3.4 Some simplifications}

Recall from (B.1) that $\Omega(c, h)$ stands for the government surplus in marginal utility terms. Use the resource constraint to write $\Omega$ as function of consumption only,

$$
\boldsymbol{\Omega}(c) \equiv \Omega(c, c+g)=\left[U_{c}(c, 1-c-g)-U_{l}(c, 1-c-g)\right] c-U_{l}(c, 1-c-g) g
$$

Differentiating with respect to consumption delivers

$$
\mathbf{\Omega}^{\prime}(c)=\Delta(c) c+\Gamma(c) g+U_{c}-U_{l}
$$

The change in the surplus of the government $\boldsymbol{\Omega}^{\prime}()$ will show up repeatedly later. We will simplify (C.36) using information from the optimal wedges. Evaluate the optimal wedges (C.33) and (C.34) at the full confidence economy $\sigma=(0,0)$ and rearrange to get

\footnotetext{
${ }^{4}$ And this optimal value would correspond to $\Phi(0)$ in our notation.
} 


$$
\begin{aligned}
& \Delta\left(c_{t}(0)\right) c_{t}(0)+\Gamma\left(c_{t}(0)\right) g_{t}=\frac{1+\Phi(0)}{\Phi(0)}\left(U_{l t}(0)-U_{c t}(0)\right) \\
& \Delta\left(c_{0}(0)\right) c_{0}(0)+\Gamma\left(c_{0}(0)\right) g_{0}-Z\left(c_{0}(0)\right) b_{0}=\frac{1+\Phi(0)}{\Phi(0)}\left(U_{l 0}(0)-U_{c 0}(0)\right) .
\end{aligned}
$$

Not that $U_{c t}(0)$ is shorthand for the evaluation of the marginal utility of consumption at the full-confidence allocation. The same interpretation holds for $U_{l t}(0)$.

Using facts (C.37) and (C.38) allows us to write $\boldsymbol{\Omega}^{\prime}$ in (C.36) as

$$
\begin{aligned}
& \Omega^{\prime}\left(c_{t}(0)\right)=\frac{U_{l t}(0)-U_{c t}(0)}{\Phi(0)}, t \geq 1 \\
& \Omega^{\prime}\left(c_{0}(0)\right)=\frac{U_{l 0}(0)-U_{c 0}(0)}{\Phi(0)}+Z\left(c_{0}(0)\right) b_{0} .
\end{aligned}
$$

\section{C.4 Consumption and labor}

Result 1. ('Consumption and labor for small doubts') The partial derivatives of consumption and labor evaluated at the full-confidence allocation are

$$
\begin{aligned}
& c_{t}^{R}(0)=h_{t}^{R}(0)=\frac{U_{l t}(0)-U_{c t}(0)}{K_{t}(0)}\left[M_{t}^{* A}(0)-\frac{\Phi^{R}(0)}{\Phi(0)}\right], t \geq 0 \\
& c_{t}^{A}(0)=h_{t}^{A}(0)=\frac{U_{l t}(0)-U_{c t}(0)}{K_{t}(0)}\left[\tilde{\xi}_{t}^{A}(0)-M_{t}^{* A}(0)-\frac{\Phi^{A}(0)}{\Phi(0)}\right], t \geq 0 .
\end{aligned}
$$

$\Phi^{i}(0), i=R, A$ stands for the derivative of the cost of distortionary taxation and $K_{t}(0)$ is defined in (C.18) and (C.19). Hence, using (C.1), we get

$$
c_{t}(\sigma)=c_{t}(0)+\frac{U_{l t}(0)-U_{c t}(0)}{K_{t}(0)}\left[\sigma_{A} \tilde{\xi}_{t}^{A}(0)+\left(\sigma_{R}-\sigma_{A}\right) M_{t}^{* A}(0)-\frac{\sigma_{R} \Phi^{R}(0)+\sigma_{A} \Phi^{A}(0)}{\Phi(0)}\right] .
$$

Note the presence of $K_{t}(0)<0$ in the determination of the partial derivatives $c_{t}^{i}(0), i=R, A$. The term $\left(U_{l}-U_{c}\right) / K$ that shows up in both expressions depends only on the consumption allocation of Lucas and Stokey at time $t$, and therefore only on the realization of the government expenditure shock $g_{t}$. Note also that if the tax rate is positive with full confidence in the model (a sufficient condition would be $U_{c l} \geq 0$ ), then $U_{l}-U_{c}<0$, and therefore $\left(U_{l}-U_{c}\right) / K>0$.

Expression (C.43) shows that the pessimistic expectation management and the paternalism

affect consumption in the way we expect. A positive innovation in debt (which increases $\tilde{\xi}_{t}^{A}(0)$ ), 
increases the tax rate and therefore reduces consumption. Furthermore, if we assume paternalism and the government doubts the model less than the household $\left(\sigma_{R}>\sigma_{A}\right)$, a positive innovation in utility (good times) is associated with less taxes (since the less pessimistic government taxes more bad times and less good times), which increases consumption. Paternalism would act in the opposite way if $\sigma_{R}<\sigma_{A}$.

Proof. From the resource constraint (49), we have $c_{t}^{i}(0)=h_{t}^{i}(0), i=R, A$. Rewrite the optimal wedge for $t \geq 1$ (C.33) as function of $\sigma$,

$$
\left(U_{l t}(\sigma)-U_{c t}(\sigma)\right)\left(1+\tilde{\xi}_{t}(\sigma)+\Phi(\sigma) \Lambda_{t}(\sigma)\right)=\Phi(\sigma) \Lambda_{t}(\sigma)\left[\Delta\left(c_{t}(\sigma)\right) c_{t}(\sigma)+\Gamma\left(c_{t}(\sigma)\right) g_{t}\right]
$$

Derivatives with respect to $\sigma_{R}$. Differentiate the left-hand side and the right-hand side of (C.44) with respect to $\sigma_{R}$ and evaluate at $\left(\sigma_{R}, \sigma_{A}\right)=(0,0)$ to get

$$
\begin{aligned}
\operatorname{LHS}^{R}(0)= & -(1+\Phi(0)) \Delta\left(c_{t}(0)\right) c_{t}^{R}(0)+\left(U_{l t}(0)-U_{c t}(0)\right)\left[\Phi^{R}(0)-\Phi(0) M_{t}^{* A}(0)\right](\mathrm{C} \\
R H S^{R}(0)= & {\left[\Phi^{R}(0)-\Phi(0) M_{t}^{* A}(0)\right]\left(\Delta\left(c_{t}(0)\right) c_{t}(0)+\Gamma\left(c_{t}(0)\right) g_{t}\right)+\Phi(0)\left[\Delta^{\prime}\left(c_{t}(0)\right) c_{t}(0)\right.} \\
& \left.+\Gamma^{\prime}\left(c_{t}(0)\right) g_{t}+\Delta\left(c_{t}(0)\right)\right] c_{t}^{R}(0)
\end{aligned}
$$

In deriving these expressions we used the result $\tilde{\xi}_{t}^{R}(0)=0$ from (C.13) and that $\Lambda_{t}^{R}(0)=$ $-N_{t}^{* R}(0)=-M_{t}^{* A}(0)$ from (C.10). Combining the two sides, collecting the terms that multiply $c_{t}^{R}(0)$ and using the definition of $K_{t}(0)$ in (C.18) delivers

$$
c_{t}^{R}(0) K_{t}(0)=\left[U_{l t}(0)-U_{c t}(0)-\left(\Delta\left(c_{t}(0)\right) c_{t}(0)+\Gamma\left(c_{t}(0)\right) g_{t}\right)\right]\left(\Phi^{R}(0)-\Phi(0) M_{t}^{* A}(0)\right)
$$

Use now (C.37) to simplify (C.47) and get (C.41).

Derivatives with respect to $\sigma_{A}$. Proceed now to differentiation of the optimal wedge with respect to $\sigma_{A}$. At $\sigma=(0,0)$ we have

$$
\begin{aligned}
\operatorname{LHS}^{A}(0)= & -(1+\Phi(0)) \Delta\left(c_{t}(0)\right) c_{t}^{A}(0)+\left(U_{l t}(0)-U_{c t}(0)\right)\left[\tilde{\xi}_{t}^{A}(0)+\Phi^{A}(0)\right. \\
& \left.+\Phi(0) M_{t}^{* A}(0)\right] \\
\operatorname{RHS}^{A}(0)= & {\left[\Phi^{A}(0)+\Phi(0) M_{t}^{* A}(0)\right]\left(\Delta\left(c_{t}(0)\right) c_{t}(0)+\Gamma\left(c_{t}(0)\right) g_{t}\right)+\Phi(0)\left[\Delta^{\prime}\left(c_{t}(0)\right) c_{t}(0)\right.} \\
& \left.+\Gamma^{\prime}\left(c_{t}(0)\right) g_{t}+\Delta\left(c_{t}(0)\right)\right] c_{t}^{A}(0),
\end{aligned}
$$


where we used (C.9). Equalize the two sides and collect terms that multiply $c_{t}^{A}(0)$ to get

$$
\begin{aligned}
c_{t}^{A}(0) K_{t}(0)= & \left(U_{l t}(0)-U_{c t}(0)\right)\left[\tilde{\xi}_{t}^{A}(0)+\Phi^{A}(0)+\Phi(0) M_{t}^{* A}(0)\right] \\
& -\left(\Phi^{A}(0)+\Phi(0) M_{t}^{* A}(0)\right)\left[\Delta\left(c_{t}(0)\right) c_{t}(0)+\Gamma\left(c_{t}(0)\right) g_{t}\right]
\end{aligned}
$$

Using (C.37) to simplify (C.50) delivers (C.42).

Initial period. The analysis above used the optimal wedge for $t \geq 1$. The initial period is different due to the possible presence of initial debt $b_{0}$. Write the optimal wedge (C.34) as function of $\sigma$,

$$
(1+\Phi(\sigma))\left(U_{l 0}(\sigma)-U_{c 0}(\sigma)\right)=\Phi(\sigma)\left[\Delta\left(c_{0}(\sigma)\right) c_{0}(\sigma)+\Gamma\left(c_{0}(\sigma)\right) g_{0}-Z\left(c_{0}(\sigma)\right) b_{0}\right] .
$$

Differentiating now with respect to $\left(\sigma_{R}, \sigma_{A}\right)$, evaluating at $\sigma=(0,0)$ and using fact (C.38) and the definition of $K_{0}(0)$ in (C.19) delivers $c_{0}^{i}(0)=-\frac{U_{l 0}(0)-U_{c 0}(0)}{K_{0}(0)} \frac{\Phi^{i}(0)}{\Phi(0)}, i=R, A$, which are the same expressions as in (C.41) and (C.42), since $M_{0}^{* A}=\tilde{\xi}_{0}^{A}=0$.

\section{C.5 Cost of distortionary taxation}

Result 2. ('Cost of distortionary taxation for small doubts')

The partial derivatives of the cost of distortionary taxation at $\sigma=(0,0)$ are

$$
\begin{aligned}
\Phi^{R}(0) & =\frac{\Phi(0) E_{0} \sum_{t=0}^{\infty} \beta^{t} z_{t}(0) M_{t}^{* A}(0)}{E_{0} \sum_{t=0}^{\infty} \beta^{t} z_{t}(0)} \\
\Phi^{A}(0) & =\frac{\Phi(0)^{2} E_{0} \sum_{t=0}^{\infty} \beta^{t} M_{t}^{* A}(0) \Omega\left(c_{t}(0)\right)+\Phi(0) E_{0} \sum_{t=0}^{\infty} \beta^{t} z_{t}(0)\left[\tilde{\xi}_{t}^{A}(0)-M_{t}^{* A}(0)\right]_{(, .53}}{E_{0} \sum_{t=0}^{\infty} \beta^{t} z_{t}(0)}
\end{aligned}
$$

where

$$
z_{t}(0) \equiv \frac{\left(U_{l t}(0)-U_{c t}(0)\right)^{2}}{K_{t}(0)}
$$

and $\boldsymbol{\Omega}($.$) defined in (C.35). Thus, we get$

$$
\Phi(\sigma)=\Phi(0)+\sigma_{R} \Phi^{R}(0)+\sigma_{A} \Phi^{A}(0)
$$


Proof. The partial derivatives $\Phi^{i}$ show up in the calculation of the approximate consumption and labor, (C.41) and (C.42). Note that, in contrast to the other derivatives that we considered, $\Phi^{i}$ are not random (because the multiplier $\Phi$ is non-stochastic). Rewrite the implementability constraint (64) as

$$
E_{0} \sum_{t=0}^{\infty} \beta^{t} M_{t}^{*}(\sigma) \boldsymbol{\Omega}\left(c_{t}(\sigma)\right)=U_{c 0}(\sigma) b_{0}
$$

Proceeding with differentiation and evaluation at $\sigma=(0,0)$, and using (C.7) and (C.8), we get

$$
\begin{array}{ll}
\sigma_{R}: & E_{0} \sum_{t=0}^{\infty} \beta^{t} \boldsymbol{\Omega}^{\prime}\left(c_{t}(0)\right) c_{t}^{R}(0)=Z\left(c_{0}(0)\right) c_{0}^{R}(0) b_{0} \\
\sigma_{A}: & E_{0} \sum_{t=0}^{\infty} \beta^{t} M_{t}^{* A}(0) \boldsymbol{\Omega}\left(c_{t}(0)\right)+E_{0} \sum_{t=0}^{\infty} \beta^{t} \boldsymbol{\Omega}^{\prime}\left(c_{t}(0)\right) c_{t}^{A}(0)=Z\left(c_{0}(0)\right) c_{0}^{A}(0) b_{0} .
\end{array}
$$

Use now expressions (C.39) and (C.40) to substitute for $\boldsymbol{\Omega}^{\prime}$ to get

$$
\begin{array}{ll}
\sigma_{R}: & E_{0} \sum_{t=0}^{\infty} \beta^{t} \frac{U_{l t}(0)-U_{c t}(0)}{\Phi(0)} c_{t}^{R}(0)=0 \\
\sigma_{A}: & E_{0} \sum_{t=0}^{\infty} \beta^{t} M_{t}^{* A}(0) \Omega\left(c_{t}(0)\right)+E_{0} \sum_{t=0}^{\infty} \beta^{t} \frac{U_{l t}(0)-U_{c t}(0)}{\Phi(0)} c_{t}^{A}(0)=0 .
\end{array}
$$

Finally, using expressions (C.41) and (C.42) for $c_{t}^{i}(0), i=R, A$ and solving for $\Phi^{i}(0), i=R, A$ delivers (C.52) and (C.53). Note that $z_{t}(0)<0$ since $K_{t}(0)<0$. So the denominator in $(\mathrm{C} .52)$ and (C.53) is negative. We need additional information on the specifics of the problem to be able to sign the numerators.

\section{C.6 Tax rate}

Result 3. ('Tax rate for small doubts')

- The tax rate is approximately equal to

$$
\tau_{t}(\sigma)=\tau_{t}(0)+\alpha_{t}(0)\left[\sigma_{A} \tilde{\xi}_{t}^{A}(0)+\left(\sigma_{R}-\sigma_{A}\right) M_{t}^{* A}(0)-\frac{\sigma_{R} \Phi^{R}(0)+\sigma_{A} \Phi^{A}(0)}{\Phi(0)}\right], t \geq 0(\mathrm{C} .57)
$$


with $\alpha_{t}(0)$ a coefficient that depends only on the current shock $g_{t}$ through the full confidence allocation. When $U_{c l} \geq 0$, we have $\alpha_{t}(0)<0$.

- In the case of the example with constant Frisch elasticity (C.23), the full confidence tax rate and the coefficient $\alpha$ are constant,

$$
\begin{aligned}
\tau_{t}(0) & =\frac{\Phi(0)\left(\gamma+\phi_{h}\right)}{1+\Phi(0)\left(1+\phi_{h}\right)} \\
\alpha_{t}(0) & =-\frac{\Phi(0)\left(\gamma+\phi_{h}\right)}{\left(1+\Phi(0)\left(1+\phi_{h}\right)\right)^{2}} .
\end{aligned}
$$

Thus, (C.57) implies that the approximate tax rate becomes a random walk with respect to $\pi$,

$$
\begin{aligned}
\tau_{t}(\sigma)-\tau_{t-1}(\sigma)= & -\frac{\Phi(0)\left(\gamma+\phi_{h}\right)}{\left(1+\Phi(0)\left(1+\phi_{h}\right)\right)^{2}}\left[\sigma_{A} \Phi(0)\left(U_{c t}(0) b_{t}(0)-E_{t-1} U_{c t}(0) b_{t}(0)\right)\right. \\
& \left.+\left(\sigma_{R}-\sigma_{A}\right)\left(V_{t}(0)-E_{t-1} V_{t}(0)\right)\right], t \geq 1
\end{aligned}
$$

Proof. Write the tax rate as $\tau_{t}(\sigma)=1-U_{l t}(\sigma) / U_{c t}(\sigma)$. Differentiating and evaluating at $(0,0)$ gives

$$
\tau_{t}^{i}(0)=\frac{U_{c c t}(0) U_{l t}(0)+U_{l l t}(0) U_{c t}(0)-U_{c l t}(0)\left(U_{c t}(0)+U_{l t}(0)\right)}{\left(U_{c t}(0)\right)^{2}} c_{t}^{i}(0), i=R, A
$$

Note that if $U_{c l} \geq 0$, the expression multiplying $c_{t}^{i}(0)$ is negative. Using now (C.41) and (C.42) we get

$$
\begin{aligned}
\tau_{t}^{R}(0) & =\alpha_{t}(0)\left[M_{t}^{* A}(0)-\frac{\Phi^{R}(0)}{\Phi(0)}\right] \\
\tau_{t}^{A}(0) & =\alpha_{t}(0)\left[\tilde{\xi}_{t}^{A}(0)-M_{t}^{* A}(0)-\frac{\Phi^{A}(0)}{\Phi(0)}\right]
\end{aligned}
$$

where

$$
\alpha_{t}(0) \equiv \frac{U_{c c t}(0) U_{l t}(0)+U_{l l t}(0) U_{c t}(0)-U_{c l t}(0)\left(U_{c t}(0)+U_{l t}(0)\right)}{\left(U_{c t}(0)\right)^{2}} \frac{U_{l t}(0)-U_{c t}(0)}{K_{t}(0)}
$$

Combining (C.61) and (C.62) and using (C.1) delivers (C.57). The coefficient $\alpha_{t}(0)$ depends only on the realization of the shock the current period $g_{t}$ through the Lucas and Stokey allocation. 
Furthermore, when $U_{c l} \geq 0$ (and thus $U_{l t}(0)<U_{c t}(0)$ ), we have $\alpha_{t}(0)<0$ as can been seen from (C.63).

Constant Frisch. At first note how the expression for $\alpha_{t}(0)$ simplifies if we have $U_{c l}=0$. Drop for simplicity the 'zero' notation and rewrite (C.63) as

$$
\begin{aligned}
\alpha & =\frac{U_{l}-U_{c}}{U_{c}} \frac{U_{c c} \frac{U_{l}}{U_{c}}+U_{l l}}{K} \\
& =-\tau \frac{U_{c c}(1-\tau)+U_{l l}}{K}
\end{aligned}
$$

where in the second line we used $\tau=1-U_{l} / U_{c}$.

Turn now to the constant Frisch case and recall that the tax rate is given by (74), which reduces to (C.58) with no doubts about the model. Use expressions (C.25) and (C.26) for period one onward to get

$$
\begin{aligned}
K & =T^{c}-T^{l}=-\left[\gamma c^{-\gamma-1}(1+\Phi(1-\gamma))+a_{h} \phi_{h} h^{\phi_{h}-1}\left(1+\Phi\left(1+\phi_{h}\right)\right)\right] \\
& \left.=-\left(1+\Phi\left(1+\phi_{h}\right)\right)\left[\gamma c^{-\gamma-1} \frac{1+\Phi(1-\gamma)}{1+\Phi\left(1+\phi_{h}\right)}+a_{h} \phi_{h} h^{\phi_{h}-1}\right)\right] \\
& =\left(1+\Phi\left(1+\phi_{h}\right)\right)\left[U_{c c}(1-\tau)+U_{l l}\right]
\end{aligned}
$$

where we used the fact that $1-\tau=\frac{1+\Phi(1-\gamma)}{1+\Phi\left(1+\phi_{h}\right)}$ (from (C.58)- recall that we simplified the notation here). Use now (C.65) and the expression for the tax rate to simplify (C.64) and get finally (C.59). Take then first differences in (C.57) and use the expressions (C.5) and (C.17) for the increments $m_{t}^{* A}(0)$ and $\eta_{t}(0)$ respectively to get (C.60).

\section{C.7 Debt}

Result 4. ('Debt for small doubts')

- Let $y_{t} \equiv U_{c t} b_{t}$ denote debt in marginal utility units. The partial derivatives are given by

$$
\begin{aligned}
& y_{t}^{R}(0)=A\left(g^{t}\right) M_{t}^{* A}(0)+B_{R}\left(g^{t}\right) \\
& y_{t}^{A}(0)=A\left(g^{t}\right)\left(\tilde{\xi}_{t}^{A}(0)-M_{t}^{* A}(0)\right)+B_{A}\left(g^{t}\right),
\end{aligned}
$$

where the respective coefficients are defined as 


$$
\begin{aligned}
A\left(g^{t}\right) \equiv & \frac{E_{t} \sum_{i=0}^{\infty} \beta^{i} z_{t+i}(0)}{\Phi(0)} \\
B_{R}\left(g^{t}\right) \equiv & E_{t} \sum_{i=0}^{\infty} \beta^{i} \frac{z_{t+i}(0)}{\Phi(0)}\left(M_{t+i}^{* A}(0)-M_{t}^{* A}(0)\right)-\frac{\Phi^{R}(0)}{(\Phi(0))^{2}} E_{t} \sum_{i=0}^{\infty} \beta^{i} z_{t+i}(0) \\
B_{A}\left(g^{t}\right) \equiv & E_{t} \sum_{i=0}^{\infty} \beta^{i} \frac{z_{t+i}(0)}{\Phi(0)}\left[\left(\tilde{\xi}_{t+i}^{A}(0)-\tilde{\xi}_{t}^{A}(0)\right)-\left(M_{t+i}^{* A}(0)-M_{t}^{* A}(0)\right)\right] \\
& -\frac{\Phi^{A}(0)}{(\Phi(0))^{2}} E_{t} \sum_{i=0}^{\infty} \beta^{i} z_{t+i}(0)+E_{t} \sum_{i=1}^{\infty} \beta^{i} m_{t+i}^{* A}(0) y_{t+i}(0)
\end{aligned}
$$

with $z_{t}(0)$ defined in (C.54). Thus, debt in marginal utility is given approximately by

$$
y_{t}(\sigma)=y_{t}(0)+A\left(g^{t}\right)\left[\sigma_{A} \tilde{\xi}_{t}^{A}(0)+\left(\sigma_{R}-\sigma_{A}\right) M_{t}^{* A}(0)\right]+\sigma_{R} B_{R}\left(g^{t}\right)+\sigma_{A} B_{A}\left(g^{t}\right) .
$$

The coefficients $A\left(g^{t}\right), B_{i}\left(g^{t}\right), i=R, A$ depend on the entire history of shocks. If the reference model $\pi$ is Markov, then the history-independence of the full-confidence allocation delivers history-independent coefficients, $A\left(g^{t}\right)=A\left(g_{t}\right), B_{i}\left(g^{t}\right)=B_{i}\left(g_{t}\right), i=R, A$.

- Let $c_{t}(\sigma)=c_{t}(0)+\sigma_{R} c_{t}^{R}(0)+\sigma_{A} c_{t}^{A}(0)$ and $y_{t}(\sigma)=y_{t}(0)+\sigma_{R} y_{t}^{R}(0)+\sigma_{A} y_{t}^{A}(0)$ denote the approximate consumption and debt in marginal units. Then, debt to first-order is given by

$$
b_{t}(\sigma)=\frac{y_{t}(\sigma)}{U_{c t}(0)}-\frac{Z\left(c_{t}(0)\right)}{U_{c t}(0)} b_{t}(0)\left(c_{t}(\sigma)-c_{t}(0)\right)
$$

where $Z($.$) is defined in (C.22).$

Proof. From the dynamic budget constraint of the government we have

$$
y_{t}(\sigma)=\Omega\left(c_{t}(\sigma)\right)+\beta E_{t} m_{t+1}^{*}(\sigma) y_{t+1}(\sigma), t \geq 1
$$

Debt in $U_{c}$ units. Differentiate (C.73), evaluate at $(0,0)$ and use (C.5) and (C.6) to get

$$
\begin{array}{ll}
\sigma_{R}: & y_{t}^{R}(0)=\Omega^{\prime}\left(c_{t}(0)\right) c_{t}^{R}(0)+\beta E_{t} y_{t+1}^{R}(0) \\
\sigma_{A}: & y_{t}^{A}(0)=\Omega^{\prime}\left(c_{t}(0)\right) c_{t}^{A}(0)+\beta E_{t} m_{t+1}^{* A}(0) y_{t+1}(0)+\beta E_{t} y_{t+1}^{A}(0)
\end{array}
$$


Solving forward we get

$$
\begin{aligned}
& y_{t}^{R}(0)=E_{t} \sum_{i=0}^{\infty} \beta^{i} \Omega^{\prime}\left(c_{t+i}(0)\right) c_{t+i}^{R}(0) \\
& y_{t}^{A}(0)=E_{t} \sum_{i=0}^{\infty} \beta^{i} \Omega^{\prime}\left(c_{t+i}(0)\right) c_{t+i}^{A}(0)+E_{t} \sum_{i=1}^{\infty} \beta^{i} m_{t+i}^{* A}(0) y_{t+i}(0)
\end{aligned}
$$

Use now (C.39), (C.41), (C.42) and the definition of $z_{t}(0)$ in (C.54) to get

$$
\begin{aligned}
& y_{t}^{R}(0)=E_{t} \sum_{i=0}^{\infty} \beta^{i} \frac{z_{t+i}(0)}{\Phi(0)}\left[M_{t+i}^{* A}(0)-\frac{\Phi^{R}(0)}{\Phi(0)}\right] \\
& y_{t}^{A}(0)=E_{t} \sum_{i=0}^{\infty} \beta^{i} \frac{z_{t+i}(0)}{\Phi(0)}\left[\tilde{\xi}_{t+i}^{A}(0)-M_{t+i}^{* A}(0)-\frac{\Phi^{A}(0)}{\Phi(0)}\right]+E_{t} \sum_{i=1}^{\infty} \beta^{i} m_{t+i}^{* A}(0) y_{t+i}(0)
\end{aligned}
$$

Use now the identities $M_{t+i}^{* A}(0)=\left(M_{t+i}^{* A}(0)-M_{t}^{* A}(0)\right)+M_{t}^{* A}(0)$ and $\tilde{\xi}_{t+i}^{A}(0)=\left(\tilde{\xi}_{t+i}^{A}(0)-\right.$ $\left.\tilde{\xi}_{t}^{A}(0)\right)+\tilde{\xi}_{t}^{A}(0)$ and rewrite (C.74) and (C.75) as (C.66) and (C.67) respectively.

Debt. Write debt in marginal utility units as $y_{t}(\sigma)=U_{c t}(\sigma) b_{t}(\sigma)$. Differentiate and evaluate at $(0,0)$ to get

$$
y_{t}^{i}(0)=Z\left(c_{t}(0)\right) b_{t}(0) c_{t}^{i}(0)+U_{c t}(0) b_{t}^{i}(0), i=R, A .
$$

Thus,

$$
b_{t}^{i}(0)=\frac{1}{U_{c t}(0)}\left[y_{t}^{i}(0)-Z\left(c_{t}(0)\right) b_{t}(0) c_{t}^{i}(0)\right], i=R, A,
$$

and therefore,

$$
\begin{aligned}
b_{t}(\sigma) & =b_{t}(0)+\sigma_{R} b_{t}^{R}(0)+\sigma_{A} b_{t}^{A}(0) \\
& =\underbrace{b_{t}(0)+\frac{1}{U_{c t}(0)}\left[\sigma_{R} y_{t}^{R}(0)+\sigma_{A} y_{t}^{A}(0)\right]}_{y_{t}(\sigma) / U_{c t}(0)}-\frac{Z\left(c_{t}(0)\right)}{U_{c t}(0)} b_{t}(0) \underbrace{\left[\sigma_{R} c_{t}^{R}(0)+\sigma_{A} c_{t}^{A}(0)\right]}_{c_{t}(\sigma)-c_{t}(0)},
\end{aligned}
$$

by realizing that $b_{t}(0)=y_{t}(0) / U_{c t}(0)$. 


\section{C.8 Formulas for Markov shocks}

Assume that the reference probability model of $g$ is a time-invariant Markov chain with transition matrix $\Pi$ of dimension $N \times N$. The Markovian assumption, together with the historyindependence of the full confidence allocation, allows us to derive convenient present-value formulas for several coefficients in the expansion. We present formulas for the case of zero initial debt, which can be easily modified to incorporate the more general case of $b_{0} \neq 0$.

Martingale increments. At first we need to calculate the increments to the martingale derivatives $M_{t}^{* A}$ and $\tilde{\xi}_{t}^{A}$. For that we need to calculate the household's expected discounted utility $V$ for the case of no-robustness and the bonds in marginal utility terms, $y \equiv U_{c} b$. We are dropping the ' 0 ' notation that indicates the full-confidence economy whenever we use vectors from now on, but remember that we are always referring to the Lucas and Stokey solution. We have

$$
\begin{aligned}
V & =(\mathbf{I}-\beta \boldsymbol{\Pi})^{-1} U \\
y & =(\mathbf{I}-\beta \mathbf{\Pi})^{-1} \Omega,
\end{aligned}
$$

where $V, y, U, \Omega$ column vectors of dimension $N$, with each element corresponding to the respective state and $\mathbf{I}$ the $N \times N$ identity matrix. ${ }^{5}$ We can derive the government debt by dividing each element of $y$ with the corresponding marginal utility $U_{c}$. Let $\mathbf{m}^{* \mathbf{A}}$ denote the matrix that collects the increments to the martingale $M_{t}^{* A}$ with corresponding element $\mathbf{m}^{* \mathbf{A}}{ }_{i j}=$ $m^{* A}(j \mid i)=V_{j}-E(V \mid i)$ and let $\boldsymbol{\eta}$ denote the matrix that collects the increments to $\tilde{\xi}_{t}^{A}$ with element $\boldsymbol{\eta}_{i j}=\eta(j \mid i)=\Phi(0)\left(y_{j}-E(y \mid i)\right)$. Given the vectors $V$ and $y$ in (C.77) and (C.78), we can write these matrices as

$$
\begin{aligned}
\mathbf{m}^{* \mathbf{A}} & =\mathbb{1} V^{T}-\Pi V \mathbb{1}^{T} \\
\boldsymbol{\eta} & =\Phi(0)\left(\mathbb{1} y^{T}-\Pi y \mathbb{1}^{T}\right)
\end{aligned}
$$

where $\mathbb{1}$ is an $N \times 1$ column vector with ones everywhere and the superscript ' $T$ ' refers to transpose. Note that the increments to the martingale derivatives inherit the Markov property. Let $\circ$ denote element-by-element multiplication between two matrices with the same dimensions (or else Hadamard multiplication) and let $0_{N \times 1}$ denote the $\mathrm{N}$-dimensional zero column vector. Note that $\left(\boldsymbol{\Pi} \circ \mathbf{m}^{* \mathbf{A}}\right) \mathbb{1}=0_{N \times 1}$ and $(\boldsymbol{\Pi} \circ \boldsymbol{\eta}) \mathbb{1}=0_{N \times 1}$, since the conditional mean of the increments is zero.

\footnotetext{
${ }^{5}$ We reserve the use of boldface for matrices in this section.
} 
Present values. We want now to calculate the discounted present values that show up in results 2 and 4 . These expressions involve expected discounted sums of products of the historydependent martingale derivatives $\left(M_{t}^{* A}\right.$ or $\left.\tilde{\xi}_{t}^{A}\right)$, or the increment $m_{t}^{* A}(0)$, with functions of the Lucas and Stokey allocation like $\Omega\left(c_{t}(0), h_{t}(0)\right)$ or $z_{t}(0)$, that are only state-dependent.

For example, consider the sum $\mathbb{S} \equiv E_{t} \sum_{i=1}^{\infty} \beta^{i} m_{t+i}^{* A}(0) y_{t+i}(0)$ that shows up in (C.70). If we expand it, we get

$$
\begin{aligned}
\mathbb{S}= & \beta \sum_{g_{t+1}} \pi\left(g_{t+1} \mid g_{t}\right) m^{* A}\left(g_{t+1} \mid g_{t}\right) y\left(g_{t+1}\right) \\
& +\beta^{2} \sum_{g_{t+1}} \pi\left(g_{t+1} \mid g_{t}\right) \sum_{g_{t+2}} \pi\left(g_{t+2} \mid g_{t+1}\right) m^{* A}\left(g_{t+2} \mid g_{t+1}\right) y\left(g_{t+2}\right) \\
& +\beta^{3} \sum_{g_{t+1}} \pi\left(g_{t+1} \mid g_{t}\right) \sum_{g_{t+2}} \pi\left(g_{t+2} \mid g_{t+1}\right) \sum_{g_{t+3}} \pi\left(g_{t+3} \mid g_{t+2}\right) m^{* A}\left(g_{t+3} \mid g_{t+2}\right) y\left(g_{t+3}\right)+\ldots \\
= & \beta e_{g_{t}}^{T}\left(\mathbf{\Pi} \circ \mathbf{m}^{* \mathbf{A}}\right) y+\beta^{2} e_{g_{t}}^{T} \boldsymbol{\Pi}\left(\mathbf{\Pi} \circ \mathbf{m}^{* \mathbf{A}}\right) y+\beta^{3} e_{g_{t}}^{T} \mathbf{\Pi}^{2}\left(\mathbf{\Pi} \circ \mathbf{m}^{* \mathbf{A}}\right) y+\ldots \\
= & \beta e_{g_{t}}^{T}\left(\mathbf{I}+\beta \boldsymbol{\Pi}+\beta^{2} \boldsymbol{\Pi}^{2}+\beta^{3} \mathbf{\Pi}^{3}+\ldots\right)\left(\mathbf{\Pi} \circ \mathbf{m}^{* \mathbf{A}}\right) y \\
= & \beta e_{g_{t}}^{T}(\mathbf{I}-\beta \mathbf{\Pi})^{-1}\left(\boldsymbol{\Pi} \circ \mathbf{m}^{* \mathbf{A}}\right) y,
\end{aligned}
$$

where $e_{g_{t}}$ be a column vector with 1 at position $i$, when $g_{t}=g_{i}$ and zero otherwise.

The case where we have multiplication with the martingale $M_{t}^{* A}(0)=\sum_{i=1}^{t} m_{i}^{* A}(0)$ is slightly more complicated. Consider for example the term $\mathbb{I} \equiv E_{0} \sum_{t=0}^{\infty} \beta^{t} M_{t}^{* A}(0) \Omega_{t}(0)$ in the numerator in $(\mathrm{C} .53)$, where $\Omega_{t}(0)$ shorthand for $\Omega\left(c_{t}(0), h_{t}(0)\right)$. II can be rewritten as

$$
\begin{aligned}
\mathbb{I}= & E_{0} \sum_{t=1}^{\infty} m_{t}^{* A}(0) \sum_{j=t}^{\infty} \beta^{j} \Omega_{j}(0)=E_{0} m_{1}^{* A}(0) E_{1}\left[\beta \Omega_{1}(0)+\beta^{2} \Omega_{2}(0)+\ldots\right] \\
& +E_{0} m_{2}^{* A}(0) E_{2}\left[\beta^{2} \Omega_{2}(0)+\beta^{3} \Omega_{3}(0)+\ldots\right]+E_{0} m_{3}^{* A}(0) E_{3}\left[\beta^{3} \Omega_{3}(0)+\beta^{4} \Omega_{4}(0)+\ldots\right]+\ldots \\
= & \beta e_{g_{0}}^{T}\left(\mathbf{\Pi} \circ \mathbf{m}^{* \mathbf{A}}\right)(\mathbf{I}-\beta \boldsymbol{\Pi})^{-1} \Omega+\beta^{2} e_{g_{0}}^{T} \mathbf{\Pi}\left(\mathbf{\Pi} \circ \mathbf{m}^{* \mathbf{A}}\right)(\mathbf{I}-\beta \mathbf{\Pi})^{-1} \Omega \\
& +\beta^{3} e_{g_{0}}^{T} \boldsymbol{\Pi}^{2}\left(\mathbf{\Pi} \circ \mathbf{m}^{* \mathbf{A}}\right)(\mathbf{I}-\beta \boldsymbol{\Pi})^{-1} \Omega+\ldots \\
= & \beta e_{g_{0}}^{T}\left(\mathbf{I}+\beta \mathbf{\Pi}+\beta^{2} \mathbf{\Pi}^{2}+\ldots\right)\left(\mathbf{\Pi} \circ \mathbf{m}^{* \mathbf{A}}\right)(\mathbf{I}-\beta \mathbf{\Pi})^{-1} \Omega \\
= & \beta e_{g_{0}}^{T}(\mathbf{I}-\beta \mathbf{\Pi})^{-1}\left(\mathbf{\Pi} \circ \mathbf{m}^{* \mathbf{A}}\right)(\mathbf{I}-\beta \mathbf{\Pi})^{-1} \Omega .
\end{aligned}
$$

We get similar expressions when the product involves the partial sum $\tilde{\xi}_{t}^{A}(0)=\sum_{i=1}^{t} \eta_{i}(0)$. For example, the term that involves $\tilde{\xi}_{t}^{A}(0)$ in the numerator of (C.53) becomes 


$$
E_{0} \sum_{t=0}^{\infty} \beta^{t} z_{t}(0) \tilde{\xi}_{t}^{A}(0)=E_{0} \sum_{t=1}^{\infty} \eta_{t}(0) \sum_{j=t}^{\infty} \beta^{j} z_{j}(0)=\beta e_{g_{0}}^{T}(\mathbf{I}-\beta \boldsymbol{\Pi})^{-1}(\boldsymbol{\Pi} \circ \boldsymbol{\eta})(\mathbf{I}-\beta \boldsymbol{\Pi})^{-1} z,
$$

where $z$ an $N \times 1$ column vector.

We repeat this type of calculations and collect the relevant expressions in the following result.

Result 5. Assume with have Markov shocks with transition matrix $\boldsymbol{\Pi}$.

- The coefficients in (C.52) and (C.53) become

$$
\begin{aligned}
\Phi^{R}(0) & =\frac{\Phi(0) e_{g_{0}}^{T} \beta(\mathbf{I}-\beta \mathbf{\Pi})^{-1}\left(\mathbf{\Pi} \circ \mathbf{m}^{* \mathbf{A}}\right)(\mathbf{I}-\beta \mathbf{\Pi})^{-1} z}{e_{g_{0}}^{T}(\mathbf{I}-\beta \boldsymbol{\Pi})^{-1} z} \\
\Phi^{A}(0) & =\frac{\Phi(0) e_{g_{0}}^{T} \beta(\mathbf{I}-\beta \boldsymbol{\Pi})^{-1}\left[\Phi(0)\left(\boldsymbol{\Pi} \circ \mathbf{m}^{* \mathbf{A}}\right)(\mathbf{I}-\beta \boldsymbol{\Pi})^{-1} \Omega+\left[(\boldsymbol{\Pi} \circ \boldsymbol{\eta})-\left(\mathbf{\Pi} \circ \mathbf{m}^{* \mathbf{A}}\right)\right](\mathbf{I}-\beta \boldsymbol{\Pi})^{-1} z\right]}{e_{g_{0}}^{T}(\mathbf{I}-\beta \boldsymbol{\Pi})^{-1} z} .
\end{aligned}
$$

- The coefficients in (C.68)-(C.70) become

$$
\begin{aligned}
A\left(g_{t}\right)= & \frac{e_{g_{t}}^{T}(\mathbf{I}-\beta \boldsymbol{\Pi})^{-1} z}{\Phi(0)} \\
B_{R}\left(g_{t}\right)= & \frac{1}{\Phi(0)} e_{g_{t}}^{T}\left[\beta(\mathbf{I}-\beta \mathbf{\Pi})^{-1}\left(\mathbf{\Pi} \circ \mathbf{m}^{* \mathbf{A}}\right)-\frac{\Phi^{R}(0)}{\Phi(0)} \mathbf{I}\right](\mathbf{I}-\beta \mathbf{\Pi})^{-1} z \\
B_{A}\left(g_{t}\right)= & \frac{1}{\Phi(0)} e_{g_{t}}^{T}\left[\beta(\mathbf{I}-\beta \boldsymbol{\Pi})^{-1}\left[(\boldsymbol{\Pi} \circ \boldsymbol{\eta})-\left(\boldsymbol{\Pi} \circ \mathbf{m}^{* \mathbf{A}}\right)\right]-\frac{\Phi^{A}(0)}{\Phi(0)} \mathbf{I}\right](\mathbf{I}-\beta \boldsymbol{\Pi})^{-1} z \\
& +e_{g_{t}}^{T} \beta(\mathbf{I}-\beta \boldsymbol{\Pi})^{-1}\left(\boldsymbol{\Pi} \circ \mathbf{m}^{* \mathbf{A}}\right) y .
\end{aligned}
$$

\section{C.9 Quasi-linear utility}

\section{C.9.1 No doubts about the model}

The utility function we are using is subcase of example (74) in proposition 4 for $\left(\gamma, a_{h}\right)=(0,1)$.

The relevant variables for $\sigma=(0,0)$ are as follows: 


$$
\begin{aligned}
\tau_{t}(0) & =\tau \equiv \frac{\Phi(0) \phi_{h}}{1+\Phi(0)\left(1+\phi_{h}\right)} \\
h_{t}(0) & =h=(1-\tau)^{\frac{1}{\phi_{h}}} \\
c_{t}(0) & =h-g_{t} \\
V_{t}(0) & =(1-\beta)^{-1}\left(h-\frac{h^{1+\phi_{h}}}{1+\phi_{h}}\right)-E_{t} \sum_{i=0}^{\infty} \beta^{i} g_{t+i} \\
b_{t}(0) & =\frac{\tau h}{1-\beta}-E_{t} \sum_{i=0}^{\infty} \beta^{i} g_{t+i} .
\end{aligned}
$$

In order to find the multiplier of the full-confidence economy $\Phi(0)$, we solve for the constant tax rate from the intertemporal budget constraint of the government:

$$
\underbrace{\tau(1-\tau)^{\frac{1}{\phi_{h}}}}_{\text {Tax revenues }}=G, \quad \text { where } \quad G \equiv(1-\beta)\left[b_{0}+E_{0} \sum_{t=0}^{\infty} \beta^{t} g_{t}\right]
$$

We assume that $G>0$, which implies that initial assets are not sufficiently large to finance government expenditures without resorting to distortionary taxes. We are looking for solutions of (C.86) at the increasing side of the Laffer curve, which implies that we are looking for $\tau<$ $\tau^{\text {Laffer }} \equiv \frac{\phi_{h}}{1+\phi_{h}}$. For a solution to exist we assume also that $G$ is less than the maximum tax revenues possible, so $G<T^{\text {Laffer }}=\frac{\phi_{h}}{\left(1+\phi_{h}\right)^{1+\frac{1}{\phi_{h}}}} \cdot{ }^{6}$ Note that if $\phi_{h}=1$, then (C.86) becomes a quadratic equation, $Q(\tau)=-\tau^{2}+\tau-G$. The root at the proper side of the Laffer curve is $\tau=\frac{1-\sqrt{1-4 G}}{2}$ since $\tau<\tau^{\text {Laffer }}=1 / 2$.

\section{C.9.2 Proof of proposition 6}

Part 1. Use (C.84) and (C.85) to get

$$
\begin{aligned}
V_{t}(0)-E_{t-1} V_{t}(0) & =b_{t}(0)-E_{t-1} b_{t}(0)=-\left(E_{t}-E_{t-1}\right) \sum_{i=0}^{\infty} \beta^{i} g_{t+i} \\
& =-\sum_{i=0}^{\infty} \beta^{i}\left(E_{t}-E_{t-1}\right) g_{t+i} \\
& =-\left(\sum_{i=0}^{\infty} \beta^{i} \gamma_{i}\right) u_{t}^{g}=-\gamma(\beta) u_{t}^{g} .
\end{aligned}
$$

\footnotetext{
${ }^{6}$ From (C.81) we see that the tax rate at the top of the Laffer curve $\tau^{\text {Laffer }}$ corresponds to $\Phi(0)=\infty$, which is excluded by not allowing $G$ to equal $T^{\text {Laffer }}$.
} 
The third line comes from the fact that given (83), we have $\left(E_{t}-E_{t-1}\right) g_{t+i}=\gamma_{i} u_{t}^{g}, i \geq 0$. Consequently, from C.5(C.7), (C.14) and (C.17) we have,

$$
\begin{aligned}
m_{t}^{* A}(0) & =n_{t}^{* R}(0)=V_{t}(0)-E_{t-1} V_{t}(0)=-\gamma(\beta) u_{t}^{g} \\
\eta_{t}(0) & =\Phi(0)\left[b_{t}(0)-E_{t-1} b_{t}(0)\right]=-\Phi(0) \gamma(\beta) u_{t}^{g} \\
M_{t}^{* A}(0) & =\sum_{i=1}^{t} m_{i}^{* A}(0)=-\gamma(\beta) \sum_{i=1}^{t} u_{i}^{g} \\
\tilde{\xi}_{t}^{A}(0) & =\sum_{i=1}^{t} \eta_{t}(0)=-\Phi(0) \gamma(\beta) \sum_{i=1}^{t} u_{i}^{g} .
\end{aligned}
$$

Part 2. Use (C.6) and (C.88) and apply the first-order expansion (C.1) to get

$$
\begin{aligned}
& n_{t}^{*}=1+\sigma_{R} n_{t}^{* R}(0)=1+\sigma_{R}\left(V_{t}(0)-E_{t-1} V_{t}(0)\right)=1+\frac{1}{\theta_{R}} \gamma(\beta) u_{t}^{g} \\
& m_{t}^{*}=1+\sigma_{A} m_{t}^{* A}(0)=1+\sigma_{A}\left(V_{t}(0)-E_{t-1} V_{t}(0)\right)=1+\frac{1}{\theta_{A}} \gamma(\beta) u_{t}^{g},
\end{aligned}
$$

by using (C.87).

Part 3. Use now (C.92) to get $E_{t} n_{t+1}^{*} u_{t+1}^{g}=E_{t} u_{t+1}^{g}+\frac{1}{\theta_{R}} \gamma(\beta) E_{t}\left(u_{t+1}^{g}\right)^{2}=\frac{1}{\theta_{R}} \gamma(\beta) \sigma_{u}^{2}$, since $E_{t} u_{t+1}^{g}=0$. Turning to the conditional variance, we have $\operatorname{Var}_{t}^{\text {Gov. }}\left(u_{t+1}^{g}\right) \equiv E_{t} n_{t+1}^{*}\left(u_{t+1}^{g}-\right.$ $\left.E_{t} n_{t+1}^{*} u_{t+1}^{g}\right)^{2}$. Treat the variance as any other function of the parameter vector $\sigma$ and expand around $(0,0)$ to get

$$
\begin{aligned}
\frac{\partial}{\partial \sigma_{i}} \operatorname{Var}_{t}^{\text {Gov. }}\left(u_{t+1}^{g}\right)_{\mid \sigma=(0,0)} & =E_{t} n_{t+1}^{* i}(0)\left(u_{t+1}^{g}-E_{t} u_{t+1}^{g}\right)^{2}-2 E_{t}\left(u_{t+1}^{g}-E_{t} u_{t+1}^{g}\right) E_{t} n_{t+1}^{* i}(0) u_{t+1}^{g} \\
& =E_{t} n_{t+1}^{* i}(0)\left(u_{t+1}^{g}\right)^{2}, i=R, A .
\end{aligned}
$$

Use (C.6) and (C.88) to get

$$
\frac{\partial}{\partial \sigma_{R}} \operatorname{Var}_{t}^{\text {Gov. }}\left(u_{t+1}^{g}\right)_{\mid \sigma=(0,0)}=-\gamma(\beta) E_{t}\left(u_{t+1}^{g}\right)^{3}, \quad \text { and } \quad \frac{\partial}{\partial \sigma_{A}} \operatorname{Var}_{t}^{\text {Gov. }}\left(u_{t+1}^{g}\right)_{\mid \sigma=(0,0)}=0
$$

which, after using (C.1), deliver the expression in (86). We can use (C.93) and perform a similar approximation for the conditional variance according to the household's worst-case beliefs, to get 


$$
\frac{\partial}{\partial \sigma_{R}} \operatorname{Var}_{t}^{\text {Hous. }}\left(u_{t+1}^{g}\right)_{\mid \sigma=(0,0)}=0, \quad \text { and } \quad \frac{\partial}{\partial \sigma_{A}} \operatorname{Var}_{t}^{\text {Hous. }}\left(u_{t+1}^{g}\right)_{\mid \sigma=(0,0)}=-\gamma(\beta) E_{t}\left(u_{t+1}^{g}\right)^{3}
$$

leading to the result stated in the proposition.

A last comment is due. The reader may wonder how the approximation of the conditional variance is related to the actual conditional variance that we would get according to the approximated beliefs in (C.92). We have

$$
\begin{array}{r}
\operatorname{Var}_{t}^{\mathrm{Gov} \cdot}\left(u_{t+1}^{g}\right)=E_{t} n_{t+1}^{*}\left(u_{t+1}^{g}\right)^{2}-\left(E_{t} n_{t+1}^{*} u_{t+1}^{g}\right)^{2} \\
\stackrel{(C .92)}{=} \underbrace{\sigma_{u}^{2}+\frac{1}{\theta_{R}} \gamma(\beta) E_{t}\left(u_{t+1}^{g}\right)^{3}}_{\text {first-order approx. }}-\frac{(\gamma(\beta))^{2}}{\theta_{R}^{2}} \sigma_{u}^{4},
\end{array}
$$

which shows that a first-order approximation of the worst-case variance around $\sigma=(0,0)$ ignores terms that are fourth-order in the reference standard deviation of the exogenous shock.

\section{C.9.3 Proof of proposition 7}

Part 1. Use formula (C.60) from result 3 for $\gamma=0$ and use also the formulas for the innovations (84) to get (87). Turning to labor, write it as $h_{t}(\sigma)=\left(1-\tau_{t}(\sigma)\right)^{\frac{1}{\phi_{h}}}$, differentiate with respect to $\sigma_{i}, i=R, A$ and evaluate at $(0,0)$ to get

$$
h_{t}^{i}(0)=-\frac{1}{\phi_{h}} \frac{h}{1-\tau} \tau_{t}^{i}(0), i=R, A
$$

where $\tau$ and $h$ were defined in (C.81) and (C.82) respectively. Then, the first-order approximation of labor is

$$
\begin{aligned}
h_{t}(\sigma) & =h-\frac{1}{\phi_{h}} \frac{h}{1-\tau}\left[\sigma_{R} \tau_{t}^{R}(0)+\sigma_{A} \tau_{t}^{A}(0)\right] \\
& =h-\frac{1}{\phi_{h}} \frac{h}{1-\tau}\left(\tau_{t}(\sigma)-\tau\right)
\end{aligned}
$$

where in the second line we use the first-order approximation for the tax rate. Evaluate now (C.96) at $t-1$, take first differences, use (87), and simplify by setting $1-\tau=\frac{1+\Phi(0)}{1+\Phi(0)\left(1+\phi_{h}\right)}$, to get (88).

The tax revenues $T_{t}(\sigma) \equiv \tau_{t}(\sigma) h_{t}(\sigma)$ have first-order derivatives 


$$
\begin{aligned}
T_{t}^{i}(0) & =\tau_{t}^{i}(0) h+\tau h_{t}^{i}(0)=h\left(1-\frac{1}{\phi_{h}} \frac{\tau}{1-\tau}\right) \tau_{t}^{i}(0) \\
& =\frac{h}{1+\Phi(0)} \tau_{t}^{i}(0), i=R, A
\end{aligned}
$$

In the first line we used (C.95) and in the second line we simplified by using the fact that $\frac{\tau}{1-\tau}=\frac{\Phi(0) \phi_{h}}{1+\Phi(0)}$ from (C.81). Thus, the first-order expansion becomes

$$
\begin{aligned}
T_{t}(\sigma) & =\tau h+\frac{h}{1+\Phi(0)}\left[\sigma_{R} \tau_{t}^{R}(0)+\sigma_{A} \tau_{t}^{A}(0)\right] \\
& =\tau h+\frac{h}{1+\Phi(0)}\left[\tau_{t}(\sigma)-\tau\right]
\end{aligned}
$$

by using again the first-order approximation of the tax rate. Take first differences in (C.98) and use (87) to get (89).

Part 2- Preliminaries. For the rest of the section recall from (C.7) and (C.14) and that the derivatives of the martingales are themselves martingales (with respect to $\pi$ ), i.e. $E_{t} M_{t+1}^{* A}(0)=$ $M_{t}^{* A}(0), E_{t} \tilde{\xi}_{t+1}^{A}(0)=\tilde{\xi}_{t}^{A}(0)$. The means are zero, $E M_{t}^{* A}(0)=E \tilde{\xi}_{t}^{A}=M_{0}^{* A}(0)=\tilde{\xi}_{0}^{A}(0)=0$.

Consider first $K$, which can be found from formula (C.65) as

$$
\begin{aligned}
K & =-\phi_{h} h^{\phi_{h}-1}\left(1+\Phi(0)\left(1+\phi_{h}\right)\right)=-\phi_{h} \frac{1-\tau}{h}\left(1+\Phi(0)\left(1+\phi_{h}\right)\right) \\
& =-\phi_{h} \frac{1+\Phi(0)}{h} \forall t \geq 0
\end{aligned}
$$

The second equality comes from using the labor supply condition, i.e. $h_{h}^{\phi}=1-\tau$ and the third equality by using the expression for the tax rate in (C.81). Thus $K$ is constant, a fact which implies that $z_{t}(0)$ in (C.54) becomes constant,

$$
\begin{gathered}
z_{t}(0)=\bar{z}=\frac{\left(U_{l}-U_{c}\right)^{2}}{K}=-\frac{\left(h^{\phi_{h}}-1\right)^{2}}{K}=\frac{\tau^{2}}{K} \stackrel{(C .99)}{=}-\frac{\tau^{2} h}{\phi_{h}(1+\Phi(0))} \\
\stackrel{(C .81)}{=}-\frac{h}{1+\Phi(0)} \frac{(\Phi(0))^{2} \phi_{h}}{\left(1+\Phi(0)\left(1+\phi_{h}\right)\right)^{2}} .
\end{gathered}
$$

Part 2 - Cost of taxation. Proceed now to the calculation of the derivatives $\Phi^{i}(0), i=R, A$ in result 2 , which are necessary for the determination of the coefficients of debt in result 4 . These 
will be greatly simplified because $z$ is constant. Consider (C.52):

$$
\Phi^{R}(0)=\Phi(0) \frac{\bar{z} E_{0} \sum_{t=0}^{\infty} \beta^{t} M_{t}^{* A}(0)}{\bar{z} /(1-\beta)}=0
$$

since $E_{0} M_{t}^{* A}(0)=M_{0}^{* A}(0)=0$. Similarly, using again the martingale property of $M_{t}^{* A}(0)$ and $\left.\tilde{\xi}_{t}^{A}(0)\right), \Phi^{A}(0)$ in $\left(\right.$ C.53) becomes $^{7}$

$$
\begin{aligned}
\Phi^{A}(0) & =\frac{(1-\beta)(\Phi(0))^{2}}{\bar{z}} E_{0} \sum_{t=0}^{\infty} \beta^{t} M_{t}^{* A}(0) \Omega_{t}(0) \\
& =-\frac{(1-\beta)(\Phi(0))^{2}}{\bar{z}} E_{0} \sum_{t=0}^{\infty} \beta^{t} M_{t}^{* A}(0) g_{t}
\end{aligned}
$$

The second line comes from the fact that $\Omega_{t}=c_{t}-(1-\tau) h=\tau h-g_{t}$. The discounted sum in (C.102) can be written as

$$
\begin{aligned}
E_{0} \sum_{t=0}^{\infty} \beta^{t} M_{t}^{* A}(0) g_{t} & =\sum_{t=1}^{\infty} \beta^{t}\left(\sum_{i=1}^{t} m_{i}^{* A}(0)\right) g_{t}=E_{0} \sum_{t=1}^{\infty} \beta^{t} m_{t}^{* A}(0) \sum_{j=0}^{\infty} \beta^{j} g_{t+j}(0) \\
\stackrel{(C .88)}{=} & -\gamma(\beta) \sum_{t=1}^{\infty} \beta^{t} \sum_{j=0}^{\infty} \beta^{j} E_{0} u_{t}^{g} g_{t+j} \\
& =-\gamma(\beta) \sigma_{u}^{2} \sum_{t=1}^{\infty} \beta^{t} \sum_{j=0}^{\infty} \beta^{j} \gamma_{j}=-\frac{\beta}{1-\beta}(\gamma(\beta))^{2} \sigma_{u}^{2}
\end{aligned}
$$

In the first line we have expanded the cumulative sum and collected terms multiplying each increment $m_{t}^{* A}(0)$. The third line comes from the fact that $E_{0} u_{t} g_{t+j}=\gamma_{j} \sigma_{u}^{2}, j \geq 0$. Use now (C.103) in (C.102) to finally get

$$
\Phi^{A}(0)=\frac{\beta \Phi(0)^{2}(\gamma(\beta))^{2}}{\bar{z}} \sigma_{u}^{2} .
$$

\footnotetext{
${ }^{7}$ Recall that $\Omega_{t}(0)$ is shorthand for $\Omega\left(c_{t}(0), h_{t}(0)\right)$ which is also equal to $\Omega\left(c_{t}(0)\right)$.
} 
Part 2 - Debt. The expressions for $y_{t}^{i}(0), i=R, A$, in result 4 are equal to $b_{t}^{i}(0), i=R, A$ since $U_{c}=1$. Using the constancy of $\bar{z}$, the martingale property and (C.101) we get

$$
\begin{aligned}
A\left(g^{t}\right) & =\frac{\bar{z}}{(1-\beta) \Phi(0)} \stackrel{(C .100)}{=}-\frac{(1-\beta)^{-1} h}{1+\Phi(0)} \frac{\Phi(0) \phi_{h}}{\left(1+\Phi(0)\left(1+\phi_{h}\right)\right)^{2}} \\
B_{R}\left(g^{t}\right) & =0 .
\end{aligned}
$$

Similarly, the expression for $B_{A}\left(g^{t}\right)$ in (C.70) simplifies to

$$
\begin{aligned}
& B_{A}\left(g^{t}\right)=-\frac{\Phi^{A}(0)}{\Phi(0)^{2}} \frac{\bar{z}}{1-\beta}+E_{t} \sum_{i=1}^{\infty} \beta^{i} m_{t+i}^{* A}(0) b_{t+i}(0) \\
& \stackrel{(C .85)}{=}-\frac{\Phi^{A}(0)}{\Phi(0)^{2}} \frac{\bar{z}}{1-\beta}+E_{t} \sum_{i=1}^{\infty} \beta^{i} m_{t+i}^{* A}(0)\left[\frac{\tau h}{1-\beta}-E_{t+i} \sum_{j=0}^{\infty} \beta^{j} g_{t+i+j}\right] \\
&=-\frac{\Phi^{A}(0)}{\Phi(0)^{2}} \frac{\bar{z}}{1-\beta}-E_{t} \sum_{i=1}^{\infty} \beta^{i} m_{t+i}^{* A}(0) \sum_{j=0}^{\infty} \beta^{j} g_{t+i+j} \\
& \stackrel{(C .88)}{=}-\frac{\Phi^{A}(0)}{\Phi(0)^{2}} \frac{\bar{z}}{1-\beta}+\gamma(\beta) \sum_{i=1}^{\infty} \beta^{i} \sum_{j=0}^{\infty} \beta^{j} \overbrace{E_{t} u_{t+i}^{g} g_{t+i+j}}^{\gamma_{j} \sigma_{u}^{2}} \\
&=-\frac{\Phi^{A}(0)}{\Phi(0)^{2}} \frac{\bar{z}}{1-\beta}+\frac{\beta}{1-\beta}(\gamma(\beta))^{2} \sigma_{u}^{2} \\
&(C .104) \quad-\frac{\beta}{1-\beta}(\gamma(\beta))^{2} \sigma_{u}^{2}+\frac{\beta}{1-\beta}(\gamma(\beta))^{2} \sigma_{u}^{2}=0 .
\end{aligned}
$$

Use now (C.105)-(C.107) in the first-order expansion (C.71) and substitute for the martingale derivatives by using (C.90) and (C.91) to get the debt policy (90) in the text. 